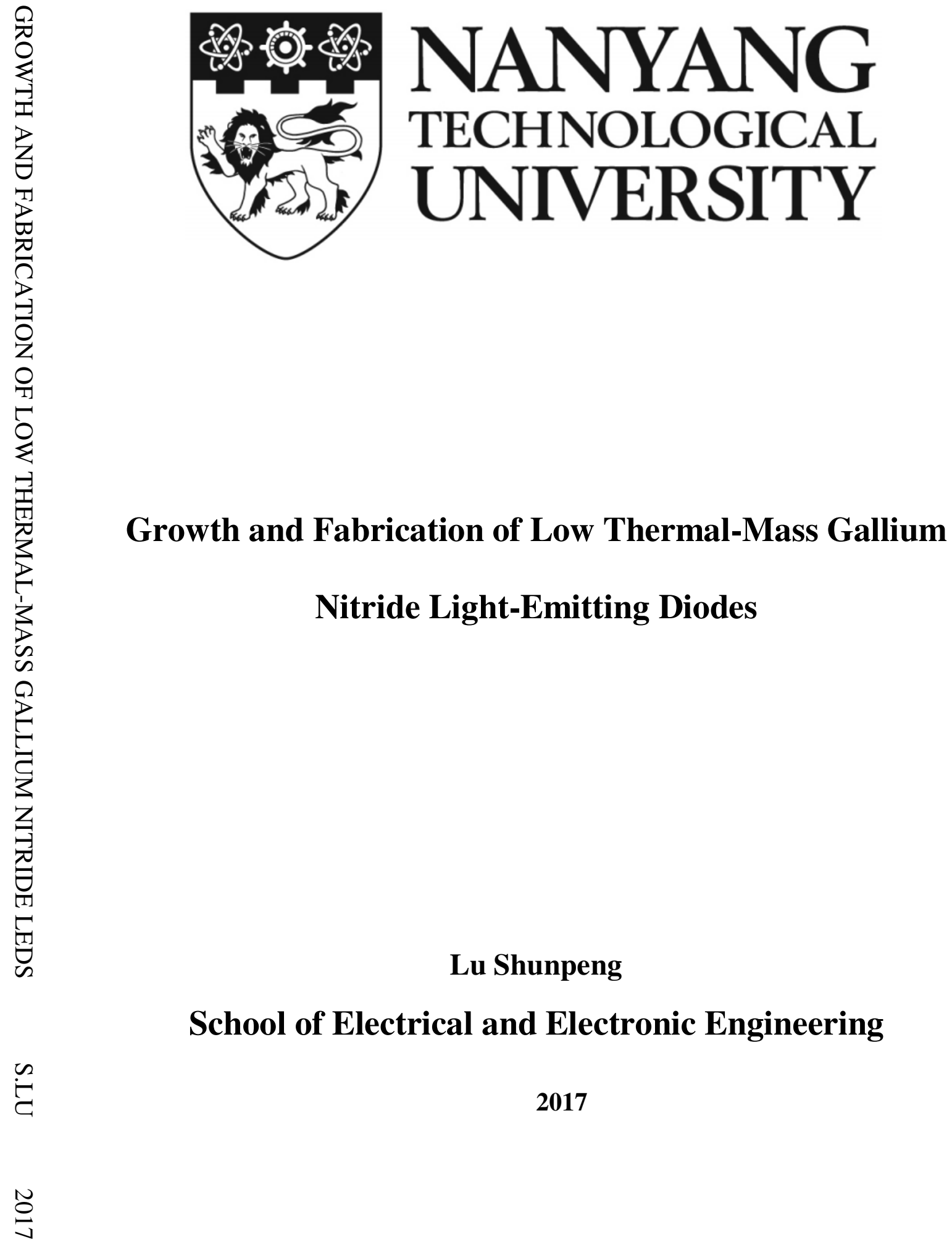





\title{
Growth and Fabrication of Low Thermal-Mass Gallium Nitride Light-Emitting Diodes
}

\author{
Lu Shunpeng
}

\section{School of Electrical and Electronic Engineering}

\author{
A thesis submitted to the Nanyang Technological University \\ In partial fulfillment of the requirement for the degree of \\ Doctor of Philosophy
}





\section{Acknowledgements}

During my PHD studies I have received much helps, instruction, and support. Here, I want to take this opportunity to show my sincere appreciation to all of those who have helped me.

Firstly, I would like to express my deep gratitude to my research supervisor, Nanyang Professor Hilmi Volkan Demir, for his enthusiastic encouragement, valuable support, and professional guidance. His useful and constructive recommendations inspired me a lot whenever I had problems with my research work and it is he who taught me how to think critically step-by-step through asking meaningful questions. I would also like to offer my special thanks to my cosupervisor, Professor Sun Xiao Wei, for his valuable and constructive suggestions during the planning and development of my work. His inspiration and precious encouragement give me lots of courage whenever I felt deflated.

I would like to thank Dr. Tian Ye Bing, from Singapore Institute of Manufacturing Technology (SIMTech), for his support and guidance in the course of my experiments.

I also want to show my particularly grateful for the assistance given by all the research staff from LUMINOUS! Center of Excellence for Semiconductor Lighting and Displays, in particular: Dr. Tan Swee Tiam, Dr. Liu Wei, Dr Kang Xue Jun, Dr Pedro Ludwig Hernández Martínez, Dr. Ju Zhen Gang, Zhang Xue Liang, Dr Zhang Zi Hui, Dr Ji Yun, Dr Zabu Kyaw, and Dr Wang Lian Cheng for their thorough discussions and precious comments during periods when I drafted the manuscript. I also would like to extend my gratitude to my project teammates Zhang Yi Ping, Zhu 
Bin Bin, Namig Hasanov, Zheng Haiyang, for their fruitful discussions, innovative inspiration, insightful critics, and comments.

In addition, I am very grateful to Ms Chia Hwee Keng Debbie and Ms Vino Prabakaran from LUMINOUS! Center of Excellence for their professional support that made my research life run more smoothly.

Finally, I am very indebted to my father (Lyu Xuanmin) and my mother (Sai Meizhi) for being very patient and supportive to walk along with me in my studying journey. Special thanks should also give to my girlfriend (Ji Jing) for her kind help and encouragement both in my research work and my daily life. 


\section{Table of Contents}

Acknowledgements ........................................................................................................... I

Table of Contents................................................................................................ III

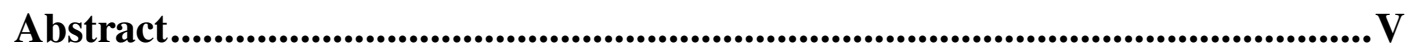

List of Figures ..................................................................................................................... IX

List of Tables .............................................................................................. XII

Abbreviations................................................................................................................... XV

Chapter 1. Introduction ...........................................................................1

1.1. Background and motivations ………………......................................

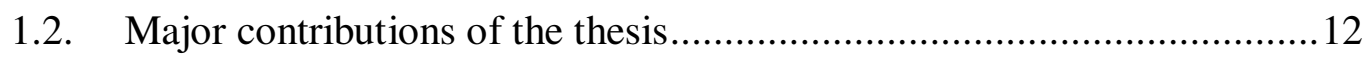

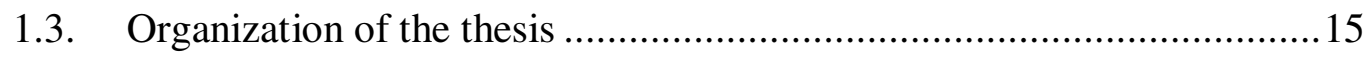

Chapter 2. Literature review ..........................................................................................17

2.1. Brief history of LEDs ......................................................................... 17

2.2. Properties of gallium nitride materials ……………………………...... 18

2.3. LED basics: band structure and efficiency …………............................20

2.4. Issues and challenges of InGaN/GaN-based LEDs for special applications. .26

Chapter 3. LED wafer growth and fabrication techniques................................32

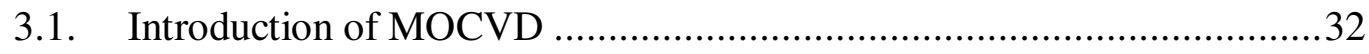

3.2. Epitaxial structure of InGaN/GaN-based LEDs.......................................35

3.3. LED fabrication techniques ………………………............................

3.4. Flip-chip and vertical LEDs ..................................................................41

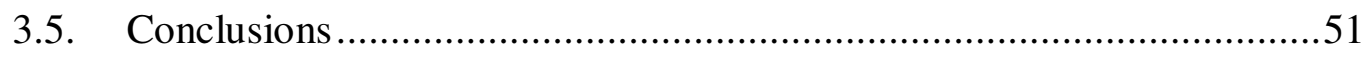

Chapter 4. Low thermal-mass LEDs: size effect and limits ..............................53 
4.1. Introduction ........................................................................ 54

4.2. Growth, fabrication and simulation of different sized LTM-LEDs .........56

4.3. Electrical and optical performances of LTM-LEDs and discussion ........58

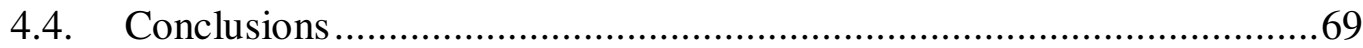

Chapter 5. Low thermal-mass LEDs: shape effects.....................................70

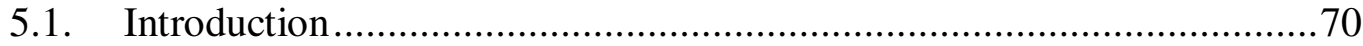

5.2. Growth, fabrication and simulations of different shaped LTM-LEDs ....73

5.3. Experimental and simulated results and discussion ..........................75

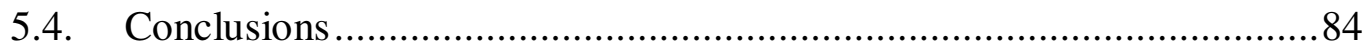

Chapter 6. Size effects on the optical properties of low thermal-mass LEDs by partitioned growth..............................................................................................86

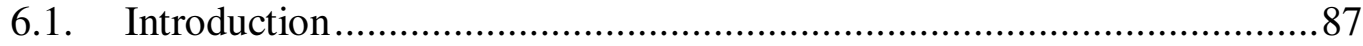

6.2. Experiments on growth and characterization.................................. 88

6.3. Experimental results of partitioned growth LTM-LEDs and discussion..89

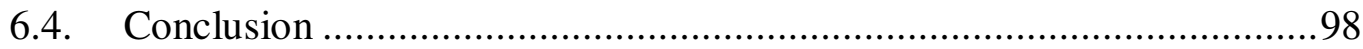

Chapter 7. Conclusions and Recommendations .............................................100

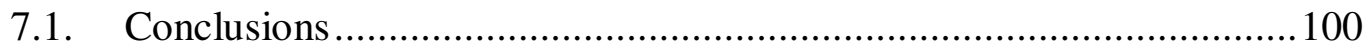

7.2. Recommendations for future work............................................. 102

Publication List ..............................................................................108

Bibliography ...............................................................................................................111 


\section{Abstract}

Over the past two decades, the technology of InGaN/GaN-based light-emitting diodes (LEDs) has made tremendous progress. The optical performance has been extensively studied to get higher luminous efficacy within a desired cost structure. Today, the efficacy of LEDs has already surpassed that of the incandescent and fluorescent luminaires. In addition, LED lighting has many advantages such as high brightness, long lifetime, high reliability, small size, high efficiency, and low power consumption. Owing to these benefits, LEDs have been widely used in a myriad of applications. Nevertheless, there is still room for improvement of the optical performance for unique applications.

For applications on mobile display backlighting, micro-displays, medical devices, visible light communication, and so on, LEDs with larger chip sizes are not effective in achieving higher power densities, higher light extraction efficiency, and faster pulsed operation even when operating at higher current densities. To fulfill the requirements of these different applications, smaller sized low thermal-mass LEDs (LTM-LEDs) are proposed in this thesis. LTM-LED has less thermal mass and better thermal conductivity which enables a lower junction temperature to be maintained. As a result of the reduced thermal mass and better thermal conductivity, LTM-LEDs can sustain much higher current density, higher power density, and faster response speed. In this dissertation, LTM-LEDs in various sizes and geometries were demonstrated and studied based on our standard flip-chip LED fabrication technique to identify the optimal power density performance. Furthermore, partitioned growth 
LTM-LEDs in different sizes are also grown by the metal-organic chemical-vapor deposition (MOCVD) system for enhanced optical output power performance.

Heat is a critical factor for the efficiency droop. Moreover, the distance between the n-contact and the LED mesa, which acts as a conductive path, has a substantial influence on current spreading and output power of LEDs, especially for the smaller sized LEDs. Hence, to increase the output power density of LTM-LEDs, it is important to reduce the thermal mass and decrease the distance between the n-contact and the LED mesa. One of the effective ways is to make LTM-LEDs smaller. Experimental results show that output power density is improved by the decreasing chip size. Our model suggests that smaller size has better uniformity on current density and also generates less heat.

As is well known, high light extraction efficiency (LEE) and better current spreading are important criteria for LEDs operating in the higher power density regime. Different shapes always show different characteristics on LEE and current spreading. To increase the power density of LTM-LEDs, triangle-, circle-, and square-shaped LTM-LEDs with the same mesa area are designed and realized in the flip-chip configuration. It was revealed that the circle-shaped LTM-LEDs show the lowest electrical and optical properties, while the triangle-shaped LTM-LEDs deliver the highest power density versus current density. However, our numerical simulations demonstrated that the LEE of triangle-shaped LTM-LEDs is only $0.06 \%$ higher than the lowest circle-shaped LTM-LEDs due to only $800 \mathrm{~nm}$ depth of the sidewalls. On the other hand, our model and simulation results show that the lower resistance at the mesa edge and the shorter $n-G a N$ current paths, which not only 
reduce the self-heating but also contribute to higher average radiative recombination rate, account for the superior performance of triangular LTM-LEDs.

The quantum-confined Stark effect (QCSE) induced by the lattice mismatch between gallium nitride and the sapphire substrate significantly hinders the optical performance of LEDs. To reduce the QCSE effect, LTM-LEDs with different sizes were grown on patterned c-plane sapphire substrate with the MOCVD technique, i.e., partitioned growth, and the size effect on the optical properties and the indium concentration for the quantum wells is studied experimentally. It is revealed that the optical properties can be improved by decreasing the chip size which subsequently reduces the in-plane compressive stress. With the decreasing chip size (from 1,000 $\mu \mathrm{m}$ to $100 \mu \mathrm{m})$, the device performance is enhanced. However, the $50 \times 50 \mu \mathrm{m}^{2}$ device shows a decreasing of output power which is attributed to more defects induced by the higher indium incorporation in the quantum wells. The underlying mechanisms of these observations are discussed based on different methods of characterization, and furthermore, it is proven that for a specific partitioned growth process, the ideal size for LTM-LEDs with the optimal power performance is identified.

In summary, both growth and fabrication techniques are used to study the LTMLEDs to improve their optical performance. Optimized shape and size for LTMLEDs with the highest optical performance are found based on certain fabrication conditions, respectively. The optimal size for partitioned growth LTM-LEDs with the highest optical power is found. This provides a good rule of thumb on how to 
choose the size and geometry to obtain LTM-LEDs with an optimal output power performance. 


\section{List of Figures}

Figure 1.1 HID versus LED lighting in a car park. ............................................. 3

Figure 1.2 The light generated per lamp and the cost per lumen changing with time [8]. 6

Figure 1.3 The applications of LEDs: (a) LED street lights on FDR Drive in midtown Manhattan, 2011 [11]; (b) LED swimming pool lights application [12]; (c) interior decoration [13]; (d) LED daytime running lights of Audi A4 [14]; (e) LED used in plant growth [15]; (f) Light Fidelity (LiFi) [16] ............................................. 8

Figure 1.4 Mobile data traffic growth forecasted by Cisco. .................................... 10

Figure 2.1 (a) The hexagonal wurtzite structure [88] and (b) the commonly used

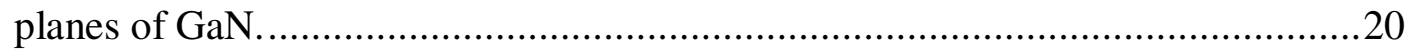

Figure 2.2 Band diagram of GaN LED structure: (a) undoped; (b) doped at equilibrium; and (c) doped at $3.2 \mathrm{~V}$ forward bias voltage.

Figure 2.3 Bandwidth versus current density graph for the $450 \mathrm{~nm}$-emitting LEDs [33]. 30

Figure 3.1 The chamber of MOCVD with planetary reactor technology [109]......33

Figure 3.2 The chamber of MOCVD with close coupled showerhead technology [110]. 34

Figure 3.3 Illustration of the MOCVD reaction process. 35

Figure 3.4 Schematic diagram of the InGaN/GaN-based LED wafer. 36

Figure 3.5 The simplified photolithography process: (a) coating photoresist on the wafer surface; (b) exposure to UV light with the photomask covering; (c) after developing in the developer. After developing, there are generally two ways to process the wafer, etching and deposition: (d) after step (c) putting the wafer into the 
dry etching chamber; (e) the etched wafer after removing the photoresist; (f) after step (c) putting the wafer into deposition chamber; (g) the metal pattern on the wafer

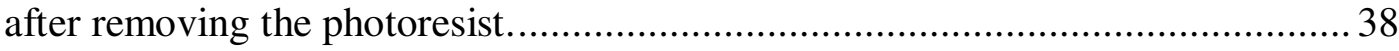

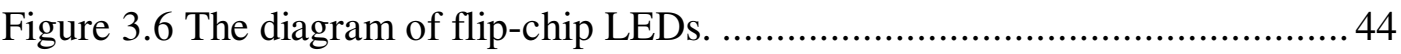

Figure 3.7 Flip-chip LED fabrication process.................................................... 45

Figure 3.8 Flip-chip LEDs: (a) Type 1 and (b) Type 2......................................46

Figure 3.9 The schematic diagram of vertical LEDs......................................... 48

Figure 3.10 Vertical LEDs lighted up at $1 \mathrm{~mA}$ : (a) Type 1(T1); (b) Type 2 (T2); (c)

Type 3 (T3); (d) Type 4 (T4) ........................................................................... 49

Figure 4.1 The microscope images of LTM-LEDs with different sizes: top-view images for (a) $25 \times 25 \mu \mathrm{m}^{2}$ (b) $50 \times 50 \mu \mathrm{m}^{2}$, (c) $100 \times 100 \mu \mathrm{m}^{2}$, (d) $200 \times 200 \mu \mathrm{m}^{2}$.

Figure 4.2 J-V diagrams of LTM-LEDs of different sizes for (a) experimental data;

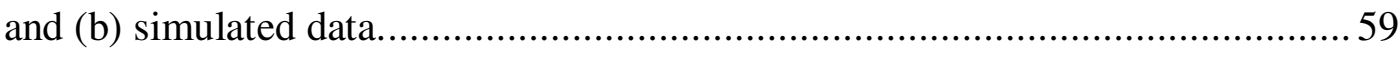

Figure 4.3 (a) Schematic current paths of the LTM-LED and (b) the simplified circuit

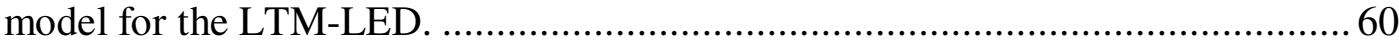

Figure 4.4 (a) $J-L$ and (b) $J-L_{0}$ behaviour at a fixed voltage bias $(11.073 \mathrm{~V}) \ldots \ldots \ldots . .63$

Figure 4.5 PD-J diagrams of LTM-LEDs in different sizes: (a) experimental results

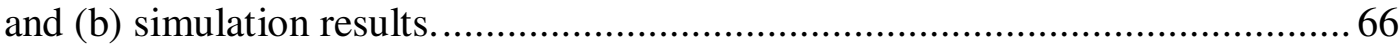

Figure 4.6 Schematic view of (a) LTM-LED current spreading layer and mesa margin and (b) the equivalent circuit model for the margin effect. 68

Figure 5.1 Experimental results of (a) $I-V$ and (b) $P-I$ diagrams of the triangular, circular, and square LTM-LEDs with the same mesa area................................... 76 
Figure 5.2 Illustration of current paths of the LTM-LEDs with a flip chip structure. 78

Figure 5.3 The microscopic pictures of (a) triangle-, (b) square-, and (c) circleshaped LTM-LEDs with the same mesa area, on which the black arrows are schematic current paths. 79

Figure 5.4 (a) $I-V$ and (b) $I-P$ characteristics of the triangular $\left(L_{2}=87.74 \mu \mathrm{m}\right)$, circular $\left(L_{2}=112.84 \mu \mathrm{m}\right)$, and square $\left(L_{2}=100 \mu \mathrm{m}\right)$ LTM-LEDs with the same area based on numerical simulation. $L_{2}$ is the longest current path from the center to the mesa edge. 81

Figure 5.5 Simulated results for the LTM-LEDs at different distances ( $x=5 \mu \mathrm{m}, x=15$ $\mu \mathrm{m}, x=25 \mu \mathrm{m}, x=35 \mu \mathrm{m}, x=45 \mu \mathrm{m}, x=65 \mu \mathrm{m}$ ) at 200mA based on Figure 5.2: (a) electron concentration; (b) hole concentration; and (c) radiative recombination rates. (d) The radiative recombination rates of the triangular, circular, and square LTMLEDs in the last quantum well (near p-GaN) as a function of the distance to the mesa edge $(x=0)$, in which $0.234 \times 1028 \mathrm{~cm}^{-3} \mathrm{~s}^{-1}, 0.18 \times 1028 \mathrm{~cm}^{-3} \mathrm{~s}^{-1}$, and $0.202 \times 1028 \mathrm{~cm}^{-}$ ${ }^{3} \mathrm{~s}^{-1}$ are the average radiative recombination rates for the triangular, circular, and square LTM-LEDs, respectively. In Figures 5.5 (a), (b), and (c), C+ indicates the growth orientation [0001] of the GaN.

Figure 6.1 The SEM images of PG LTM-LEDs with different sizes: top-view images for (a) $50 \times 50 \mu \mathrm{m}^{2}$, (c) $100 \times 100 \mu \mathrm{m}^{2}$, (e) $200 \times 200 \mu \mathrm{m}^{2}$, (g) $500 \times 500 \mu \mathrm{m}^{2}$, and (h) $1,000 \times 1,000 \mu \mathrm{m}^{2}$ chips; $45^{\circ}$ tilted view images for (b) $50 \times 50 \mu \mathrm{m}^{2}$, (d) $100 \times$ $100 \mu \mathrm{m}^{2}$, and (f) $200 \times 200 \mu \mathrm{m}^{2}$ chips.

Figure 6.2 (a) The power versus current and (b) the EQE versus current of PG LTMLEDs in different sizes. 93

Figure 6.3 Raman spectrum of $\mathrm{E}_{2}$ (high) phonon peaks for different sized PG LTMLEDs. 95 
Figure 6.4 EL spectra of PG LTM-LEDs of different sizes at 20mA: (a) the 1,000 $\times$ $1,000 \mu \mathrm{m}^{2}, 500 \times 500 \mu \mathrm{m}^{2}$, and $200 \times 200 \mu \mathrm{m}^{2}$ devices; (b) the $200 \times 200 \mu \mathrm{m}^{2}, 100$

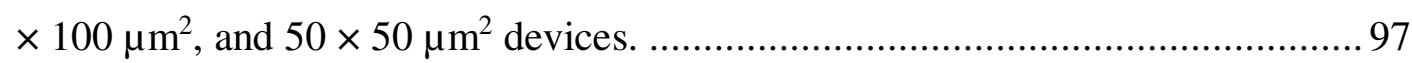

Figure 6.5 I-V characteristics for PG LTM-LEDs of different sizes. .................... 98

Figure 7.1 Schematic diagram of (a) planer sapphire substrate and (b) patterned

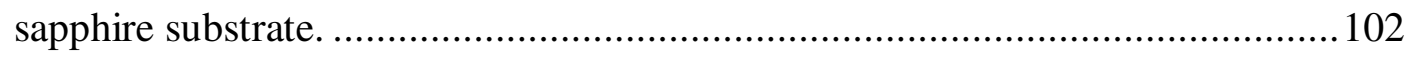

Figure 7.2 Bandwidth versus current density graph for the $450 \mathrm{~nm}$-emitting LEDs

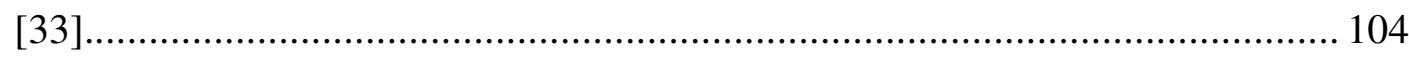

Figure 7.3 Simulated results of (a) $J-V$ characteristics of and (b) IQE\&PD-J characteristics of flip-chip versus vertical LTM-LEDs where the p-GaN is fully covered with metal and heat model is considered............................................... 105

Figure 7.4 The schematic current paths of (a) flip-chip LEDs; and (b) vertical LEDs. 106

Figure 7.5 Vertical LEDs with (a) flat n-GaN surface and (b) regular patterned n-

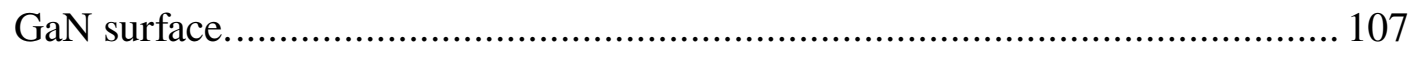




\section{List of Tables}

Table 1.1 LED versus other lighting technologies [3] ....................................... 2

Table 1.2 Comparison among different technologies for micro-displays [24]....... 11

Table 3.1 The experimental results of the bias voltage and power of flip-chip LEDs.

Table 3.2 The experimental results of bias voltage and power of four type vertical LEDs. 51

Table 5.1 LEE of triangle-, circle-, and square-shaped LTM-LEDs based on MonteCarlo methods. .75

Table 6.1 The in-plane compressive stress of different sized PG LTM-LEDs.......95 


\section{Abbreviations}

AFM

CCS

$\mathrm{Cp} 2 \mathrm{Mg}$

CRI

EBL

EL

EQE

FWHM

GSG

ICP

IQE

LEDs

LEE

$\mathrm{LiFi}$

LOP

LTM-LEDs

MFC

MOCVD

MOVPE

MQW

OMCVD

OMVPE
Atomic Force Microscope

Closed Coupled Showerhead

Bis(cyclopentadienyl)magnesium

Color Rendering Index

Electron Blocking Layer

Electroluminescence

External Quantum Efficiency

Full Width Half Maximum

Ground-Signal-Ground

Inductively Coupled Plasmas

Internal Quantum Efficiency

Light-Emitting Diodes

Light Extraction Efficiency

Light Fidelity

Light Output Power

Low Thermal-Mass LEDs

Mass Flow Controller

Metal-Organic Chemical Vapor Deposition

Metal-Organic Vapor Phase Epitaxy

Multiple Quantum Well

Organometallic Chemical Vapor Deposition

Organometallic Vapor Phase Epitaxy 


$\begin{array}{ll}\text { PG } & \text { Partitioned Growth } \\ \text { PL } & \text { Photoluminescence } \\ \text { PSS } & \text { Patterned Sapphire Substrate } \\ \text { QCSE } & \text { Quantum-Confined Stark Effect } \\ \text { QW } & \text { Quantum Well } \\ \text { RCLEDs } & \text { Resonant-Cavity LEDs } \\ \text { RIE } & \text { Reactive Ion Etching } \\ \text { RTA } & \text { Rapid Thermal Annealing } \\ \text { SEM } & \text { Scanning Electron Microscopy } \\ \text { SIMTech } & \text { Singapore Institute of Manufacturing Technology } \\ \text { SRH } & \text { Shockley-Read-Hall } \\ \text { TEGa } & \text { Triethylgallium, Ga(CH2CH3)3 } \\ \text { TEM } & \text { Trimethylindium, In(CH3)3 } \\ \text { TMAl } & \text { Trimethylalumium, Al(CH3)3 } \\ \text { TMIn } & \text { Trimetholion }\end{array}$




\section{Chapter 1. Introduction}

\subsection{Background and motivations}

Energy saving is increasingly more important and emergent technology nowadays as traditional energy sources are running out and environmental problems have drawn increasing attention. In the field of general illumination, lighting takes up about one fifth of total electricity consumption [1]. Among those sources of artificial illumination, two of the most widely used types are fluorescent and incandescent lamps. Those sources of artificial illumination have high energy consumption as their energy efficiency is low. Nevertheless, this situation is changing thanks to the development of the high-power LEDs. In practice, LEDs have already achieved much higher luminous efficacy than any other light sources. From Table 1.1, on the market, among the light sources commercially available, we can see that the LED light source had the luminous efficacy higher than $160 \mathrm{~lm} / \mathrm{W}$ in 2013, which was running in second place and just a little lower than the low pressure sodium light source. However, this situation has changed since March 2014. Cree Inc. even achieved $303 \mathrm{~lm} / \mathrm{W}$ in the laboratory [2], which is much higher than that of the incandescent and fluorescent luminaires. 
Table 1.1 LED versus other lighting technologies [3].

\begin{tabular}{|l|c|c|c|}
\hline \multicolumn{1}{|c|}{ Light source } & CRI & Lumens/watt & $\begin{array}{c}\text { Lifetime in hours } \\
\text { (x1000) }\end{array}$ \\
\hline High pressure sodium & 30 & $60-120$ & $10-24$ \\
\hline Low pressure sodium & 5 & 200 & $10-24$ \\
\hline Mercury vapour & 50 & 50 & 10 \\
\hline Metal halide & $70-95$ & $60-100$ & $6-20$ \\
\hline Fluorescent & $60-90$ & $40-100$ & $6-45$ \\
\hline CFL & $60-90$ & $50-75$ & $6-15$ \\
\hline Incandescent & $90-100$ & $5-25$ & 1 \\
\hline Induction & $50-90$ & $60-90$ & 100 \\
\hline LEP & $50-90$ & $60-90$ & 100 \\
\hline LED & $70-90$ & Up to 160 & $100+$ \\
\hline
\end{tabular}

High energy efficiency is important for LED lighting, but that is not the whole story. The more reasonable efficiency of a luminaire should also include light utilization which cares more about how well the light reaches the target area and provides appropriate illumination. For any kind of lighting application, achieving required illuminance levels using less light means higher utilization efficiency. For high power LEDs, their smaller size makes their light direction easier to control. Thus, LED lighting has a better light distribution on the target area, which reduces the total light needed from a lamp. LED outdoor applications have shown high utilization efficiency compared with traditional light fixtures. Figure 1.1 shows an example of this. From Figure 1.1 we can see that the car park area lighted by the Cree LED luminaires is brighter and more uniform. This will significantly benefit the safety for pedestrians and drivers. What is more, the total power of all the LEDs used in the car park is only $6.5 \mathrm{KW}$, which is significantly less than the $19.1 \mathrm{KW}$ of HID headlamps. 


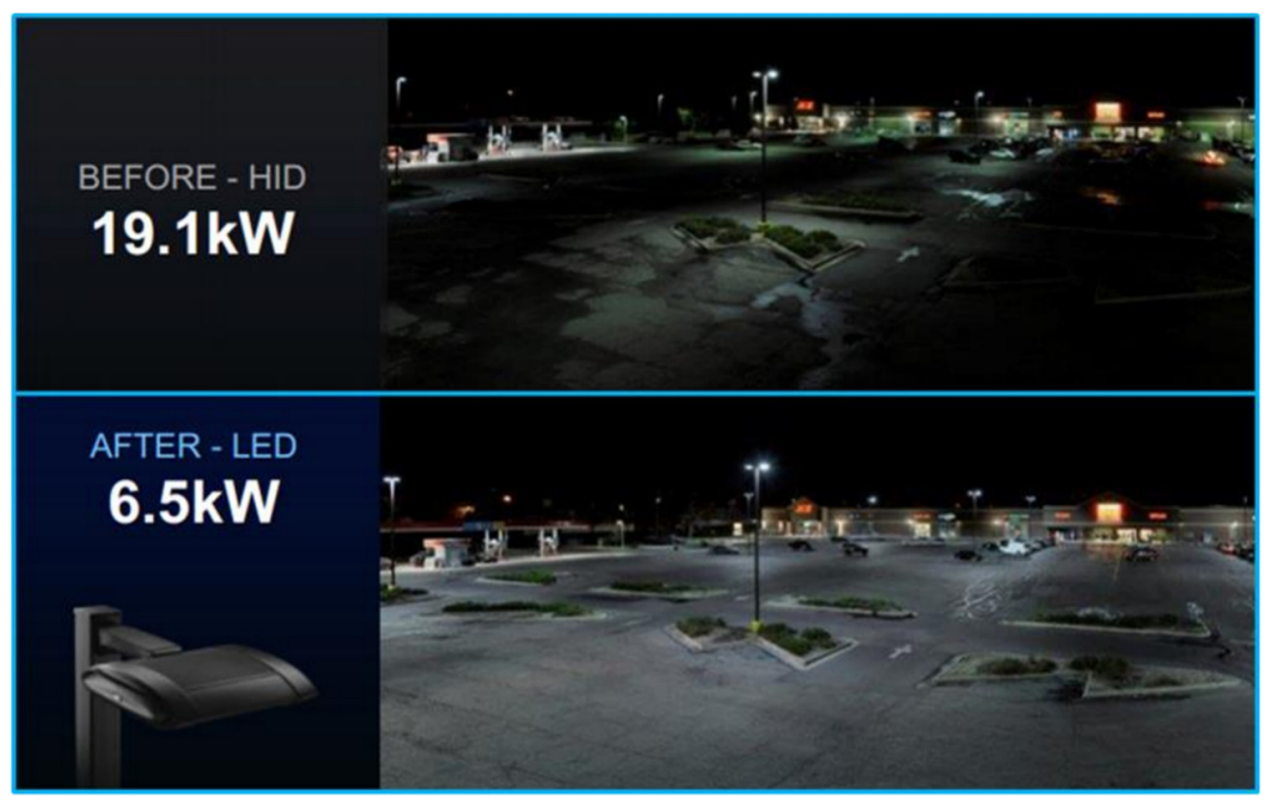

Figure 1.1 HID versus LED lighting in a car park.

Source: John Edmond, Cree Inc., SSL R\&D Workshop, San

Francisco, CA, January 2015 [4].

Because of the high energy efficiency and high utilization efficiency, LEDs have high potential for energy saving and are also environmental friendly. In the United States of America, from 2000 to 2020, \$100 billion will be saved as a result of using high power LEDs instead of conventional lighting devices. In the year 2020 it is estimated that lighting will maybe only consume half as much electricity as it did in 2000. From an environmental aspect, the amount of electricity being saved during one year reduces the carbon emission by 28 billion kilograms [1]. What is more, LEDs can also benefit our environment by reducing the use of critical, scarce, toxic, and/or energy intensive materials, such as $\mathrm{Pb}, \mathrm{Hg}$, and $\mathrm{Cd}$ [5]. Therefore, LEDs are likely to be the most environmentally friendly lighting candidates. 
Besides energy saving and environmental friendliness, LED luminaires have many advantages compared to conventional fluorescent and incandescent lamps, these are:

1) Efficiency: LEDs are the most energy saving light sources as in principle every electron can be transferred into a photon.

2) Color: There already exist blue, red, and green LEDs. In principle, we can get any color of light by mixing these three color LEDs. Compared with traditional light sources, these are more effective as no additional filters are needed to obtain any particular color. Thus, it is more efficient and lower cost.

3) Size: The millimeter-scale size (or even micrometer-scale) of the LED chips allows them to be embedded in most of mobile and portable devices and luminaries. On the other hand, those of incandescent and fluorescent light sources are generally in the range of ten centimeters.

4) Response time: LEDs can be instantly turned on or off, without noticeable delay. The rise and fall time can be of several nanoseconds. On the other hand, the response time for an incandescent lamp is several milliseconds and that of a fluorescent lamp is several seconds.

5) Cool light: Compared with most of other light sources, LEDs radiate very little infrared, which is very important for heat sensitive objects.

6) Lifetime: LEDs have the longest lifetime as a light source. It is reported that the useful life of a LED is about 50,000 hours [6]. The lifetime of fluorescent tubes is about 10,000 to 15,000 hours and that of incandescent bulbs is about 1,000 to 2,000 
hours. This extended LED lifetime reduces the maintenance costs for indoor and outdoor applications.

7) Shock resistance: LEDs are solid state components that are covered with a plastic shell, which makes them robust compared to incandescent and fluorescent lamps that are housed in glass envelopes.

The advantages mentioned above provide LED light sources a high potential for substituting fluorescent and incandescent light sources.

In order for LEDs to be commercialized, high performance and low cost are essential. Hence, as with other solid state technologies, LEDs are getting better performance and less expensive according to a predictable curve, which is governed by Haitz's law (Figure 1.2). In 1999, through charting the historical prices of LEDs and projecting forward, Roland Haitz estimated that the quantity of light generated in every LED package would increase 20 times per decade, while the cost per lumen would drop 10 times correspondingly [7, 8]. In the past two decades, there has been a substantial improvement in the efficiency of InGaN/GaN-based white LEDs. For example, LED manufacturer Cree Inc. reported that in May 2011 a LED power efficacy of $231 \mathrm{~lm} / \mathrm{W}$ was achieved in R\&D [9]. Then, in February 2013, this record was updated to $276 \mathrm{~lm} / \mathrm{W}$ [10] and recently, in March 2014 they even achieved 303 $\mathrm{lm} / \mathrm{W}$ [2] which is much higher than that of the incandescent and fluorescent luminaires. 


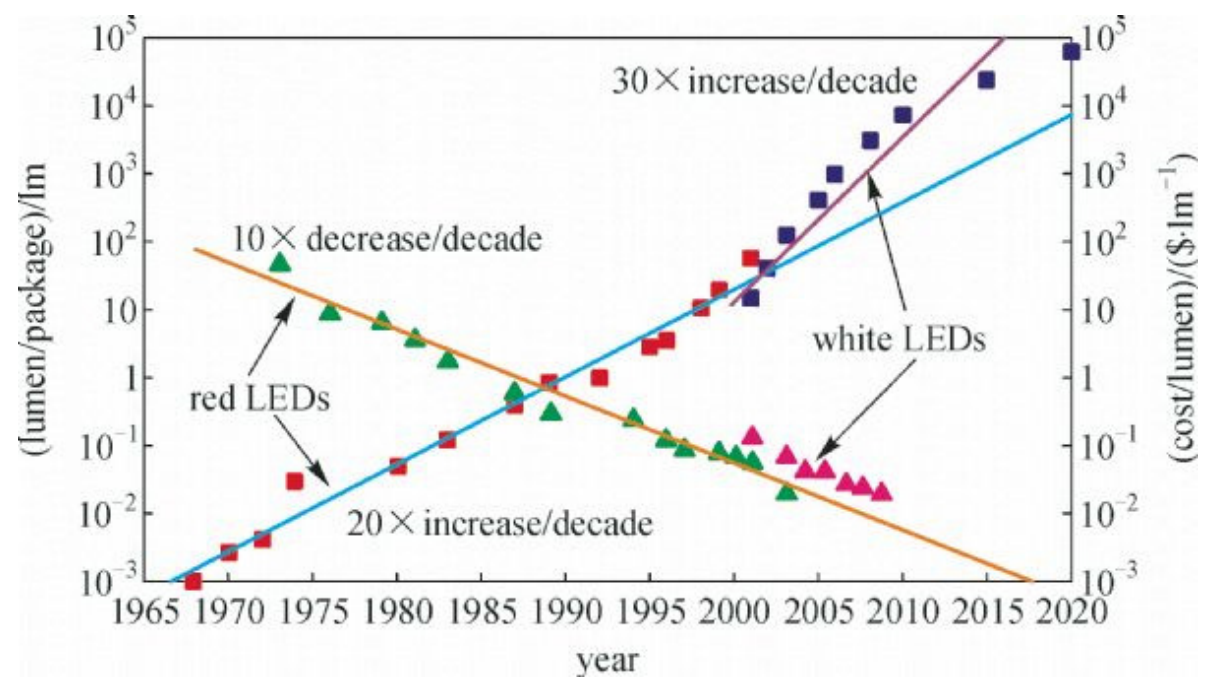

Figure 1.2 The light generated per lamp and the cost per lumen changing with time [8].

LEDs are used widely in the lighting market due to the continuous performance improvement and cost reduction. In addition to the general illumination (indoor lighting), LEDs are designed accordingly for various unique applications (as shown in Figure 1.3):

1) Street lights: White LEDs are widely used for street lights thanks to energy efficiency, high performance, and environmental sustainability.

2) Landscape lights: Including interior decoration, architectural ornament, tourist attractions decoration, etc. Those decorative LEDs are mainly used for commercial lighting, places of interest, streets, bridges, commercial buildings, public places of entertainment, community, courtyard, lawn, etc.

3) Automotive lights: In recent years, LEDs are becoming the main light component for the third brake light, steering lamp, tail light, and side light. 
4) Back lights: LEDs are suitable for backlighting, such as LCD televisions, laptop displays as they can make those screens thinner, and as the light source for digital light processing projectors because they can make those screens thinner.

5) Traffic lights and large screen display: LEDs are becoming the main component for traffic lights and are being used for lighting and displays at airports and stadiums - most of the modern stadiums are equipped with LEDs.

6) Aquarium lights: To maintain optimal aquarium temperatures, LEDs are very suitable for aquarium lighting, especially for reef aquariums as the LED is a cool light source.

7) Communications: LEDs have very fast modulation characteristics, so they can be used in communications such as light fidelity (LiFi).

8) Horticulture Lightings: To encourage plants to grow better, LEDs are used to increase photosynthesis. Plant growers prefer LEDs to other light sources because they can provide the optimum light wavelength for plant growth while emitting less heat.

9) Special lightings: Because LEDs have properties of anti-knock, robustness, moisture resistivity, small volume, low thermal radiation, and light weight, they are widely used for military equipment. The lack of infrared radiation, makes LEDs ideal for medical lighting where infrared-radiation can be harmful. 

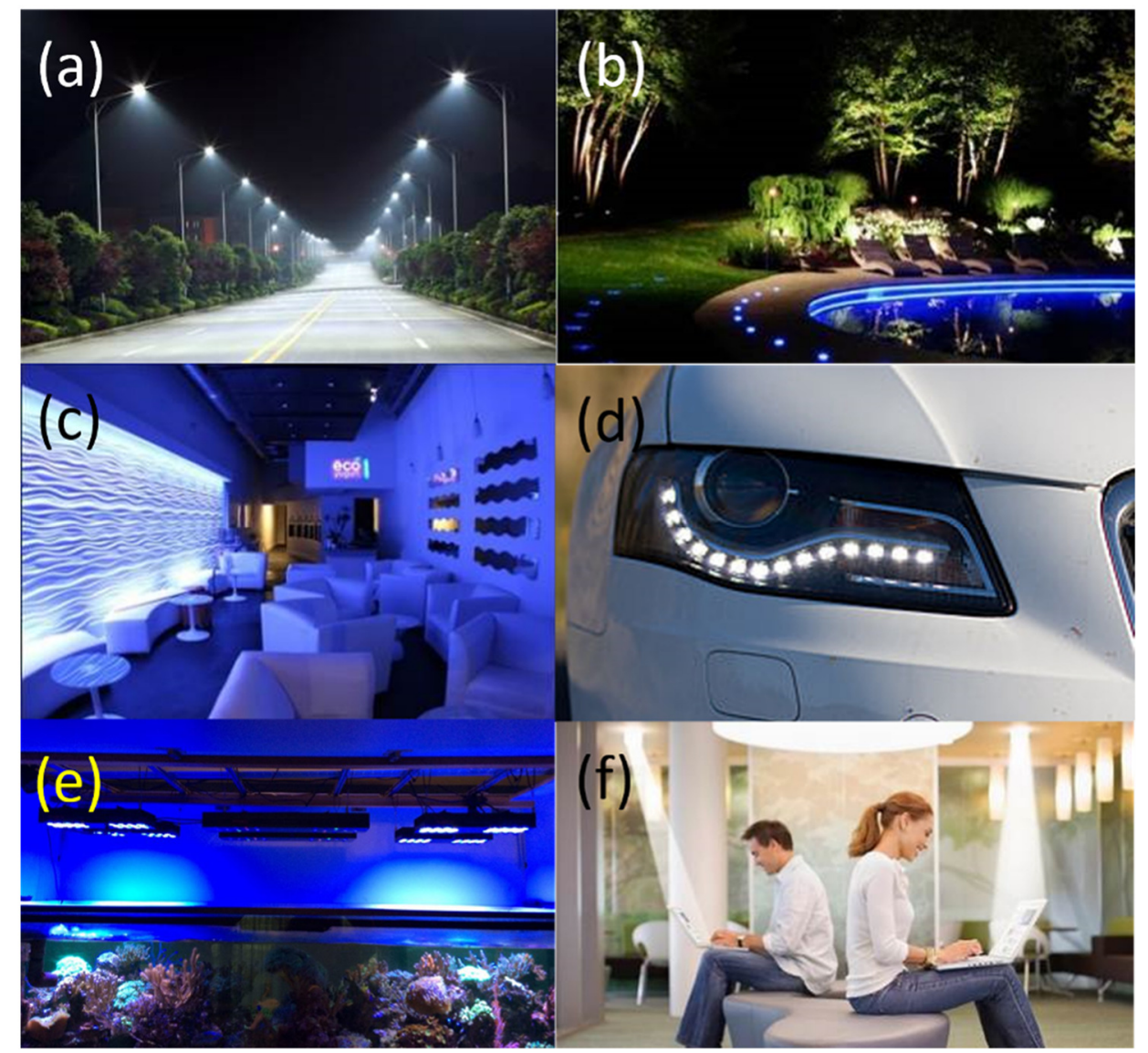

Figure 1.3 The applications of LEDs: (a) LED street lights on FDR

Drive in midtown Manhattan, 2011 [11]; (b) LED swimming pool lights application [12]; (c) interior decoration [13]; (d) LED daytime running lights of Audi A4 [14]; (e) LED used in plant growth [15]; (f) Light Fidelity (LiFi) [16].

From the above applications we can see that LEDs have a huge potential for becoming a "smart" light source, which means they can offer controllability of their spatial, temporal, spectral, color temperature, and polarization properties. In the past two decades smart LEDs have attracted much attention in both research fields and our daily life [17-19]. For example, for the aspect of spectral control and tuning, 
LEDs are the most outstanding luminaires [20], which is discussed previously for the advantages of LEDs in chapter 1. Some LED products with tunable light spectrum emission function are already available in the commercial sales market, such as the one used at the entertainment and decoration industry. However, to mix the RGB color uniformly, RGB LEDs should be placed as close as possible to each other. Thus, making LEDs smaller is a good choice to satisfy this demand. From this point of view, low thermal-mass LEDs (LTM-LEDs) which have the characteristics of micrometer size, high current density, high power density, and high response speed, are more suitable for such applications.

In fields other than illumination such as communication, LEDs can be used to transfer data or information in indoor locations and communicate with other wireless devices. LED on and off response can be very fast as GaN blue LEDs have a very short carrier lifetime. Thus, it is believed that in the near future, LED-based light fidelity $(\mathrm{LiFi})$ will play an important role in communication with the continuing rapid increase in global mobile data traffic. Cisco pointed out that global mobile data traffic of 2019 will be ten times greater than that of 2014, as shown in Figure 1.4. Therefore, to enhance the communication bandwidth, LEDs including white LEDs with phosphors, RGB white LEDs, and micro blue LEDs are intensively studied both at universities and research centers. Compared with broad area LEDs, smaller sized LEDs have higher response speed. Literature shows that the optical 3-dB modulation bandwidth of a typical GaN blue micro-LED already exceeds $245 \mathrm{MHz}$ [21]. Since LTM-LEDs can deliver high response speed and high power density, they are a better choice for visible light communication. 


\section{7\% CAGR 2014-2019}

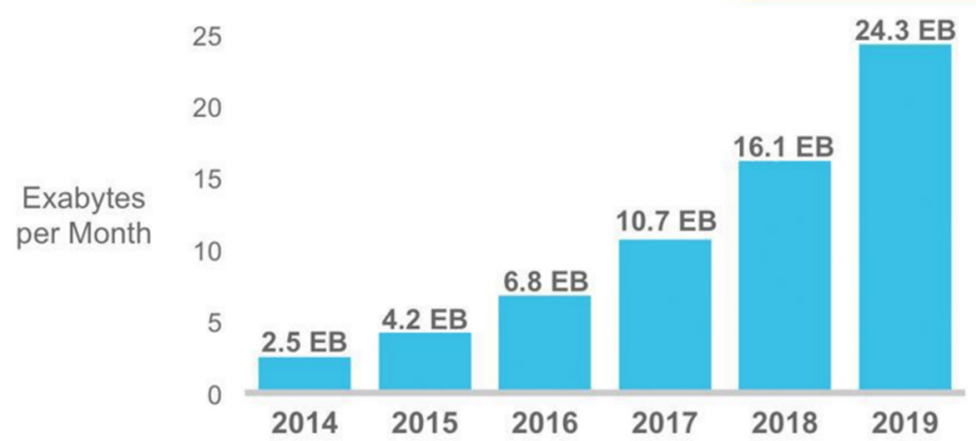

Figure 1.4 Mobile data traffic growth forecasted by Cisco.

Source: Cisco Visual Networking Index: Global Mobile Data Traffic

Forecast Update 2014-2019 White Paper, February 2015 [22].

In the field of displays, micro-displays fabricated from III-nitride based LTMLEDs have high potential for providing excellent performance due to the unique properties of these LEDs such as long lifetime, low power consumption, high-speed, wide viewing angle, high shock resistance, full-color spectrum capability, and high resolution. Micro-displays are very small high-resolution displays that can be projected or viewed with the help of mirrors or lenses. Under the help of projection optics, the pictures on the screen can be enlarged 21 times [23]. That is, a 1 inch high resolution micro-display can provide an image as large as 21 inches with the help of a lens. Thus, micro-displays can be used for TVs and computer screens as well as the electronic watch screen. This makes micro-displays suitable for highly mobile and hands-free applications such as computing, military, entertainment, firefighting, law enforcement, medical, and so on. High-resolution micro-displays are currently being intensively studied [24-27]. 
Table 1.2 Comparison among different technologies for microdisplays [24].

\begin{tabular}{lll}
\hline \hline \multicolumn{1}{c}{ Technology } & \multicolumn{1}{c}{ Liquid crystal } & \multicolumn{1}{c}{ Organic LED } \\
\hline Mechanism & Backlighting/LED & Self-emissive \\
Luminous efficacy & Medium & Low \\
Luminance & $3000 \mathrm{~cd} / \mathrm{m}^{2}$ (full color) & $1500 \mathrm{~cd} / \mathrm{m}^{2}$ (full color) \\
& $\sim 10^{4} \mathrm{~cd}^{2} \mathrm{~m}^{2}$ (green) & $\sim 10^{3} \mathrm{~cd} / \mathrm{m}^{2}$ (yellow) \\
Contrast ratio & $200: 1$ (intrinsic) & Very high $>10000: 1$ \\
Response time & $\mathrm{ms}$ & $\mu \mathrm{s}$ \\
Operating & 0 to $60^{\circ} \mathrm{C}$ requires & -50 to $70^{\circ} \mathrm{C}$ \\
temperature & heater & \\
Shock resistance & Low & Medium \\
Lifetime & Medium & Medium \\
Cost & & Low \\
\hline \hline
\end{tabular}

\begin{tabular}{|c|c|c|}
\hline III-Nitride $\mu$ LED & Digital light processing & Laser beam steering \\
\hline Self-emissive & Backlighting/LED & Backlighting/LD \\
\hline $\begin{array}{l}\text { High } \\
\sim 10^{5} \mathrm{~cd} / \mathrm{m}^{2} \text { (full color) } \\
\sim 10^{7} \mathrm{~cd} / \mathrm{m}^{2} \text { (blue } / \text { green) }\end{array}$ & $\begin{array}{l}\text { High } \\
\sim 1000 \mathrm{~cd} / \mathrm{m}^{2} \text { (full color) }\end{array}$ & $\begin{array}{l}\text { High } \\
\sim 1000 \mathrm{~cd} / \mathrm{m}^{2} \text { (full color) }\end{array}$ \\
\hline Very high $>10$ 000:1 & High & High \\
\hline ns & $\mathrm{ms}$ & $\mathrm{ms}$ \\
\hline-100 to $120^{\circ} \mathrm{C}$ & To be determined & To be determined \\
\hline High & Medium & Medium \\
\hline Long & $\begin{array}{l}\text { Medium (limited by } \\
\text { MEMS) }\end{array}$ & $\begin{array}{l}\text { Short (limited by } \\
\text { laser diodes) }\end{array}$ \\
\hline Low & High & High \\
\hline
\end{tabular}

With the development of LED technology, LTM-LEDs, which have a smaller size than broad area LEDs, have high potential for a wide variety of applications. One of the reasons for this is that the lumens per watt have rapidly increased in the last decade, and the highest LED efficacy is already beyond $300 \mathrm{~lm} / \mathrm{W}$ [2]. Hence, the LEDs need to be designed smaller to fit the brightness and balance the light 
uniformity in different applications, such as the display backlighting for cell phones, computers, and TVs. Another reason is that broad-area LEDs are not effective in achieving higher light extraction efficiency [28],[29], higher power densities [30],[31], and faster pulsed operation [29] even when operating at higher current densities. Therefore, LTM-LEDs have been proposed, which are deemed more appropriate for such applications including micro-displays [32] and visible communications [33], which typically need LEDs to operate in the current density range beyond $1,000 \mathrm{~A} / \mathrm{cm}^{2}$.

With LEDs becoming smaller, the size and shape effects on LTM-LEDs as well as the underlying physics become essential and need to be systematically studied. To understand why smaller-sized LEDs can sustain higher current densities and deliver higher power densities, LEDs of different sizes have been previously reported [34]. On the other hand, compared with the etching down method, the partitioned growth method, which uses $\mathrm{SiO}_{2}(100 \mathrm{~nm})$ as the mask to grow the epitaxial layers in the open areas on the sapphire substrate (more details can be found in chapter 6), is another important way for making LTM-LEDs. However, the size effect on the strain release of the partitioned growth is still seldom studied.

\subsection{Major contributions of the thesis}

The epitaxial wafers used throughout this dissertation were grown using the metal-organic chemical-vapor deposition (MOCVD) system. To get high crystal quality wafers with uniform thickness, the growth conditions, such as the growth rate, 
the inlet gases ratio, temperature, pressure, and doping concentration have been well optimized. Thereafter, using micro-fabrication technique, broad area LEDs and LTM-LEDs are fabricated. The ohmic contact is realized between $\mathrm{p}-\mathrm{GaN}$ and the mirror, which is a metal layer with high reflectance. The electrode pad is optimized to reduce the resistance and improve the current spreading. For the grown epitaxial LED wafers and fabricated wafers, both chip-level and wafer-level characterizations have been used to evaluate the optical and electrical properties. Based on the optimized epitaxial growth, device fabrication, and standard characterization methods, novel structures have been designed and studied to get the optimized optical power performance.

1. In our fabrication process, flip-chip LEDs and vertical LEDs were designed and comparatively studied. For the flip-chip LEDs, the $250 \mathrm{~nm}$ silver-based mirror $(\mathrm{Ni} / \mathrm{Ag} / \mathrm{Ni} / \mathrm{Au})$ was made and found to be sufficient for the $\mathrm{p}-\mathrm{GaN}$ current spreading. The results further demonstrate that to get an optimal performance, lower n-pad resistance should be reached. For the vertical LEDs, it is suggested that current blocking layer under the n-electrode is effective for improving the current uniformity and hence enhance the optical output power. Keeping a large active light emission area is found to be important for improving the optical performance.

2. Smaller-sized LEDs with the mesa area from $200 \times 200 \mu \mathrm{m}^{2}$ down to $25 \times 25 \mu \mathrm{m}^{2}$ have been comparatively studied. These smaller-sized LEDs usually have lower self-heating and better thermal dissipation characteristics due to more effective sidewall heat radiation compared with those traditional broad-area LEDs and 
hence are named as "Low Thermal-mass LEDs" (LTM-LEDs). LTM-LEDs not only have low thermal effect, but also have higher external quantum efficiency (EQE) than traditional broad-area LEDs at high current density due to better thermal management and more uniform current spreading. Here, EQE is the ratio of the photon number emitted from the LED to the electron number passing through the device. What is more, we find the optimal size for LTM-LEDs to get the optimal power density, which will have a significant impact in the future LEDs applications. In addition, we provide a model to explain why smaller-sized LEDs have higher current density and power density.

3. New structured LTM-LEDs are proposed and achieved through epitaxial growth and microfabrication. The LED wafers are grown on c-plane double polished sapphire substrates by MOCVD, then fabricated into LTM-LEDs with novel patterns. The triangle-, circle-, and square-shaped LTM-LEDs are comparatively studied both theoretically and experimentally. It is demonstrated that triangleshaped LTM-LEDs have better thermal, electrical, and optical performance. The lower resistance at the mesa edge, the shorter $\mathrm{n}-\mathrm{GaN}$ current paths and higher average radiative recombination rates are found to be the underlying reason for the superior performance of the triangle shape. This is significant for the future development of LTM-LEDs for choosing proper geometries.

4. The quantum-confined Stark effect (QCSE) induced by spontaneous and piezoelectric polarization fields limits the optical performance of LEDs. To reduce the QCSE, square-shaped LTM-LEDs in different sizes were grown on patterned c-plane sapphire substrate with $\mathrm{SiO}_{2}$ as mask. The size effect on the 
optical properties and the indium concentration for the quantum wells was studied experimentally. It was revealed that the optical properties can be improved by decreasing the chip size, which can correspondingly reduce the inplane compressive stress. However, when the size decreases to $50 \times 50 \mu \mathrm{m}^{2}$, the optical performance decreases, which is attributed to more defects induced by the higher indium incorporation in the quantum wells.

\subsection{Organization of the thesis}

This research work is organized as follows: first, the background, motivation, and major contributions are described in Chapter 1. Chapter 2 briefly discusses the historical development of LEDs, the material properties of gallium nitride, the basic band structure, internal quantum efficiency of GaN LEDs, and the challenges faced for developing the InGaN/GaN-based LED applications. Chapter 3 starts with the introduction of the epitaxial growth and describes the major equipment used in the fabrication process. Next, we discuss the comparison studies on the chip design of flip-chip LEDs and vertical LEDs. Based on the growth technique and fabrication technique, the epitaxial LED wafers are grown and then fabricated into LTM-LEDs. Chapter 4 elaborates on the size effect and LTM-LEDs limitations. Here, it is proven that for a specific flip-chip fabrication process, the ideal size for LTM-LEDs with optimal power density performance is identified. Through the shape effect study, Chapter 5 shows that the triangle-shaped LTM-LEDs deliver the best electrical and optical performance. To further increase the output power of LTM-LEDs, partitioned 
growth LTM-LEDs are proposed to restrain the QCSE. Chapter 6 presents the partitioned growth LTM-LEDs realized in different sizes and $100 \mu \mathrm{m}$ is demonstrated to be the optimal size to exhibit highest output power due to the balance between the reduced strain and the increasing of defects density. Finally, Chapter 7 provides a conclusion on the work done in this dissertation and concludes with recommendations for further study. 


\section{Chapter 2. Literature review}

\subsection{Brief history of LEDs}

Since the LEDs were invented, LED technology has made tremendous progress [35-39]. LEDs have played an extremely important part in a myriad of applications $[1,17,21,32,40-45]$ as they have many advantages such as high brightness, long lifetime, high reliability, small size, high efficiency, low energy consumption, and high-speed response. LEDs, which have the highest efficacy compared to other light sources, can in theory convert nearly all electricity into light. However, the development of LEDs has not been smooth. LEDs were first discovered by accident in 1907 [46]. For a long time, LEDs did not receive much attention and were rediscovered in the nineteen twenties and in the nineteen fifties [47]. Until the nineteen sixties, to demonstrate the semiconductor laser, groups working at General Electric Corporation, MIT Lincoln Laboratories, and IBM Corporation put more effort on the III-V materials [35, 48-51] and the LEDs, which were by-products, became commercially viable. For a long time, LEDs were only used as indicators as the output power was low. In the nineteen nineties, Nakamura et al. proposed an efficiently implemented method to obtain p-type GaN through high-temperature annealing [52]. After that, LEDs are believed to be the most promising source for illumination and blue LEDs become the hottest research area [53]. With the fast development of InGaN/GaN-based LED technology, a wide range of applications are expected. Nevertheless, there is still much room for improvement, such as the 
reliability [54-56], decreasing the efficiency droops [57-60], and increasing the light extraction $[28,40,61]$. The process never stops and great technological progress is currently being made.

\subsection{Properties of gallium nitride materials}

Gallium nitride (GaN), a compound III/V wide band gap semiconductor, has attracted intensive attention due to its wide applications. As a result of its direct bandgap, GaN is commonly used in high-power light emitting fields, such as highpower blue LEDs [62, 63], UV LEDs [64, 65], and long-lifetime violet-laser diodes [66-68]. Shuji Nakamura, Isamu Akasaki, and Hiroshi Amano were awarded the 2014 Nobel Prize in Physics for their contribution on the blue LEDs. Furthermore, $\mathrm{GaN}$ is also an excellent material for high frequency and high-voltage devices owing to its wide band gap of $3.4 \mathrm{eV}$, such as high-speed field effect transistors [69-71] and UV detectors [72-74]. Like other group III nitrides, it is insensitive to ionizing radiation which makes it a very good material for solar cells for space applications $[75,76]$.

Proper substrates are very important for high quality GaN epitaxial layers. In industry, MOCVD is the most popular method to deposit GaN epitaxial layers. The most widely adopted substrate is sapphire in virtue of low price and relative smaller lattice constant mismatch with $\mathrm{GaN}$ [77-79]. SiC is another substrate used in industry thanks to its lattice constant similar with that of $\mathrm{GaN}[79,80]$. Silicon, which is the most popular and most mature semiconductor material also draws much attention. Even though the lattice constant of $\mathrm{Si}$ is larger than that of $\mathrm{GaN}$, growing $\mathrm{GaN}$ on 
the Si substrate is one of the hottest areas of research nowadays because of the promising future that LEDs can be integrated with other mature semiconductor devices on Si substrate [81-83]. As for the GaN substrate, it is the most promising one, nevertheless it is very expensive to get large area high quality c-plane bulk GaN substrates $[84,85]$. To date, growing $\mathrm{GaN}$ on a $\mathrm{GaN}$ substrate is mainly conducted at research centers or universities.

To make InGaN/GaN-based LEDs, n-type and p-type GaN are needed. N-type $\mathrm{GaN}$ is usually made by doping with silicon ( $\mathrm{Si}$ ) and p-type $\mathrm{GaN}$ is by doping with magnesium (Mg). However, when $\mathrm{Ga}$ is substituted by $\mathrm{Si}$ or $\mathrm{Mg}$, tensile stresses are introduced and the crystal quality is reduced $[86,87]$. GaN compounds are also likely to have a high dislocation density, which is usually on the order of $10^{8} / \mathrm{cm}^{2}$ to $10^{10}$ $/ \mathrm{cm}^{2}$.

For our wafer growth, GaN is used to grow InGaN/GaN-based LED epitaxy on sapphire substrate. InGaN and $\mathrm{AlGaN}$ are always introduced into the epitaxial wafer $[89,90]$. This epitaxial wafer is always grown on $\mathrm{C}+[0001]$ orientation of the hexagonal wurtzite structure (Figure 2.1). As the III-Nitrides material has spontaneous polarization, there are spontaneous polarization charges at the layer surfaces. In addition, as III-Nitrides are piezoelectric materials and the lattice constants of $\mathrm{GaN}$ and $\mathrm{InGaN}$ are different, there are piezoelectric polarization charges in the InGaN/GaN multiple quantum wells due to the strain between each layer. These spontaneous and piezoelectric polarization charges will bring in internal electric fields, which will degrade the electrical and optical performance of LEDs 
$[60,91]$. In chapter 6 , the strain effect is discussed to find out the optimum size for the partitioned growth LEDs.
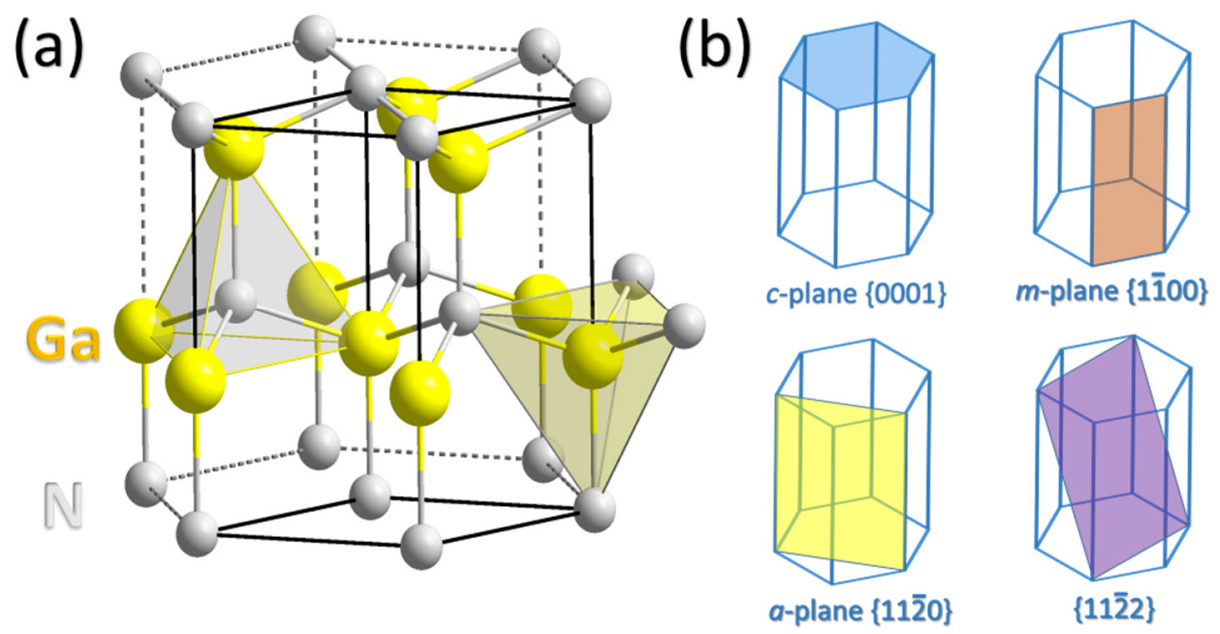

Figure 2.1 (a) The hexagonal wurtzite structure [88] and (b) the commonly used planes of GaN.

\subsection{LED basics: band structure and efficiency}

\section{$>$ Band structure of LEDs used in this thesis}

The basic structure for LEDs is the P-N junction based on inorganic semiconductors [62]. The basic components of LEDs include an n-doped electroncarrying layer and a p-doped hole-carrying layer. Similar to other diodes, when forward bias voltage is applied, current will flow from the p-side to the n-side, in other words, from anode to cathode. Electrons meet holes in the junction area between the n-doped layer and the p-doped layer, also referred to as the active region, and photons are emitted. This is radiative recombination, in which an electron in the 
conduction band falls into the valence band and emits a photon. The photon's wavelength is determined by the energy gap of the semiconductor/material. For InGaN/GaN-based high power LEDs, there are multiple quantum wells (MQWs) working as the active region between p-type gallium nitride ( $\mathrm{p}-\mathrm{GaN})$ and $\mathrm{n}$-type gallium nitride ( $\mathrm{n}-\mathrm{GaN}$ ) to enhance the radiative recombination [92]. In some structures, there is also an n-type electron blocking layer (n-EBL) between n-GaN and MQWs or a p-type electron blocking layer (p-EBL) between p-GaN and MQWs, or both in order to decrease the current leakage [54].

In our structure, we use n-GaN, MQWs, p-EBL, and p-GaN as our basic LED structure [56]. Figure 2.2 shows the band diagram obtained from the APSYS simulation of the GaN LED structure. Figure 2.2(a) is the band diagram without doping and polarization field. In this condition, there are only intrinsic electrons and holes in GaN. Figure 2.2(b) is the doped band structure (electrons concentration = $5 \times 10^{18} \mathrm{~cm}^{-1}$; hole concentration $=3 \times 10^{17} \mathrm{~cm}^{-1}$ ) at zero bias voltage. In this equilibrium condition, the electrons in $\mathrm{n}-\mathrm{GaN}$ and holes in $\mathrm{p}-\mathrm{GaN}$ are confined by the high energy barrier. The electrons should have very high energy to get to the p$\mathrm{GaN}$ region through the barrier and similarly for the holes. Figure 2.2(c) is the doped band structure at $3.2 \mathrm{~V}$ forward bias voltage. Because the barrier is flattened by this forward bias voltage, electrons and holes will diffuse toward each other side as the electron density and the hole density are very high in n-GaN and p-GaN, respectively. As there are many quantum wells between $\mathrm{n}-\mathrm{GaN}$ and $\mathrm{p}-\mathrm{GaN}$, the electrons and holes will get into the wells and recombine to emit light. This process 
when electrons and holes recombine and generate photons is named radiative recombination.
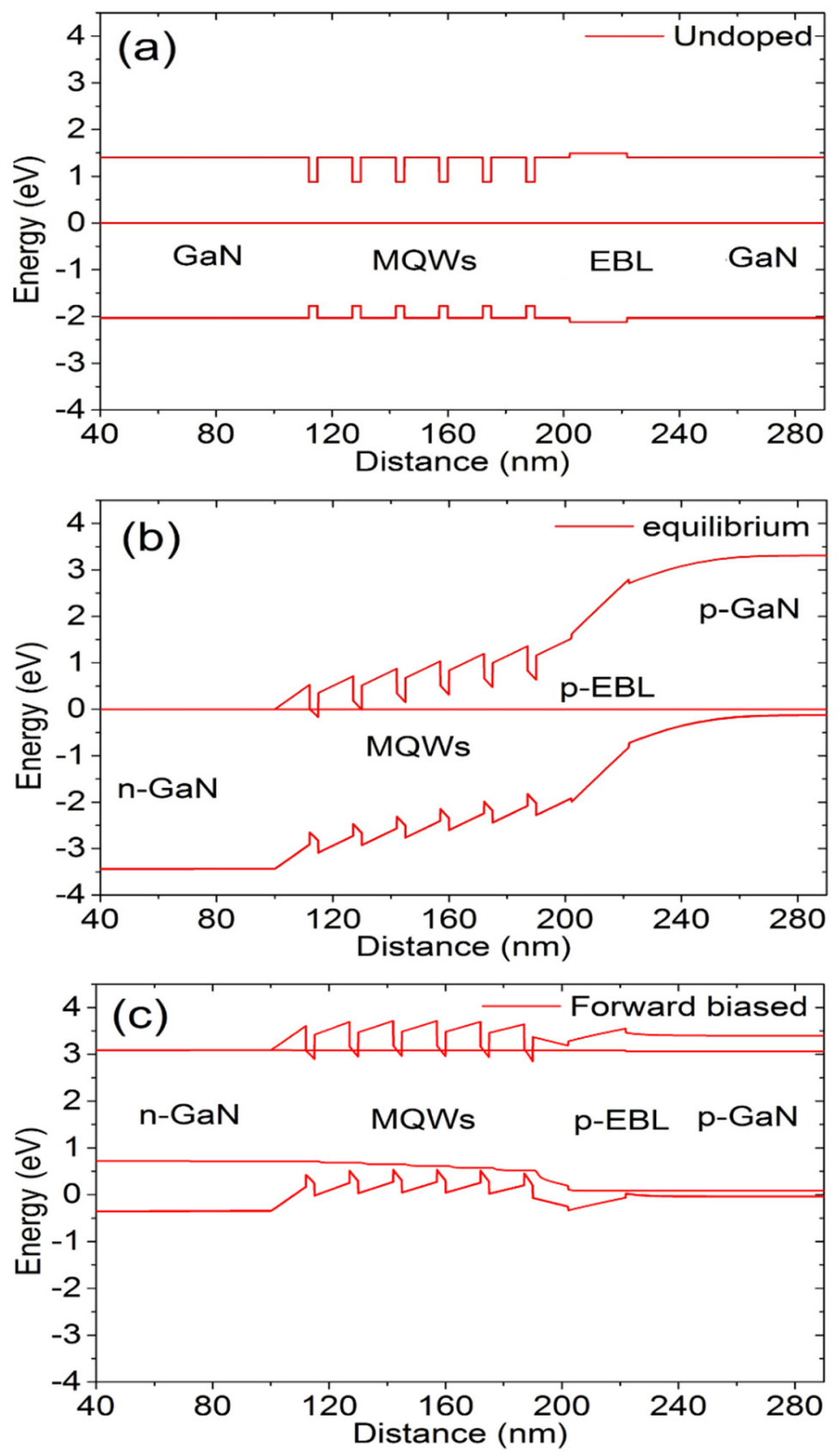

Figure 2.2 Band diagram of GaN LED structure: (a) undoped; (b) doped $\left(\right.$ electrons concentration $=5 \times 10^{18} \mathrm{~cm}^{-1}$; hole concentration $=$ $3 \times 10^{17} \mathrm{~cm}^{-1}$ ) at equilibrium; and (c) doped at $3.2 \mathrm{~V}$ forward bias 
voltage.

\section{$>$ Internal quantum efficiency of LEDs}

In the InGaN/GaN-based LEDs, the radiative recombination is the main process for the photon generation. As photons have very little momentum, the spontaneous radiative recombination is remarkable only in direct bandgap materials. For the radiative electron-hole spontaneous recombination, there are generally two types; the photoluminescence (PL) which is light emission from emitting species after the absorption of photons, and the electroluminescence (EL) which is the phenomenon of emitting light in response to an electrical current or a strong electric field.

In the GaN semiconductor LED materials, there are two types of free carriers, the electron and the hole. Without external stimuli, the product of the electron and hole concentrations is equal to the square of the intrinsic carrier concentration [93]. The relationship is as follows:

$$
n_{0} p_{0}=n_{i}^{2}
$$

where $n_{0}$ and $p_{0}$ are the electron and hole concentrations at equilibrium, and $n_{i}$ is the intrinsic carrier concentration. However, when it comes to the non-equilibrium condition, stimulated either by photons or by the injection current, the electron and hole concentration will increase. The spare electrons and holes will try to recombine to get the equilibrium state. The recombination rate $R$ can be expressed as follows [94]:

$$
R=-\frac{d n}{d t}=-\frac{d p}{d t}=B n p
$$


This is the bimolecular rate equation, in which $n$ is the electron concentration, $p$ is the hole concentration at the stimulated state, respectively; and $B$ is the recombination coefficient. Usually, $B$ is in the range of $10^{-11}$ to $10^{-13} \mathrm{~cm}^{3} / \mathrm{s}$ for direct band gap III-V semiconductors [93].

Besides the radiative recombination, Shockley-Read-Hall (SRH) recombination is another important recombination process in LEDs. The SRH recombination, which is also called trap-assisted recombination, is caused by deeplevel traps. The deep-level traps provide a new energy state between the conduction band and the valence band. The electrons can first drop to this localized state and then fall to the valence band, instead of dropping to the valence band from the conduction band directly. These deep-level traps are introduced through defects or impurities, which provide energy levels lying far from the edge of the band gap, in the lattice. For the indirect bandgap materials such as silicon, the momentums of the carriers in the lowest conduction band and the highest valence band are different. This momentum difference makes it difficult for the generation and recombination of the electron hole pair. However, this momentum difference can be absorbed by the localized state, so the SRH recombination becomes the main process in these indirect bandgap materials. In direct bandgap materials, under some conditions, the SRH recombination can also be the main process. For example, at very low current level the electrons can drop to the localized state by generating a phonon. For InGaN/GaN-based LEDs, it is essential to suppress the SRH recombination to improve the IQE, so it is important to reduce the defects in the InGaN/GaN-based LED epitaxial layer growth process [95]. 
Another important recombination process is Auger recombination. In this process, the electron hole pairs recombine and transfer the energy to a third carrier instead of emitting a photon. After getting the energy, the third carrier has higher energy level and this energy level is unstable, it will give up the excess energy as a form of thermal vibrations. This three-particle interaction is very weak at low current density and becomes more significant with increasing of current density. Normally, researchers relate this process with the efficiency droop [96-98].

Similar to radiative recombination, the recombination probability increases with the increasing of electron and hole concentration. The Auger recombination rates for electron-hole-hole process and electron-electron-hole process are proportional to $p^{2} n$ and $n^{2} p$, respectively [93]. The formulas are as follows:

$$
\begin{gathered}
R_{E}=C_{n} p n^{2} \\
R_{H}=C_{p} n p^{2}
\end{gathered}
$$

in which $R_{E}$ is the Auger recombination rate for electron-electron-hole process, $R_{H}$ is the Auger recombination rate for electron-hole-hole process, and $C_{n}$ and $C_{p}$ stands for the Auger coefficients for electron-electron-hole process and electron-hole-hole process, respectively.

In all of the above mentioned recombination processes, only the radiative recombination process contributes to light emission. When all the electron hole recombination is the radiative recombination, the internal quantum efficiency (IQE) can reach its maximum. Here, the internal quantum efficiency (IQE) $\eta$ is the number of photons emitted at active region per second over the number of electrons injected into LED per second. Having a high IQE is very critical for high power LEDs. 
However, in real applications, the efficiency always droops with increasing current. Efficiency droop is the phenomenon where the luminous efficiency of LEDs increases with current and reaches a maximum value at few to tens of milli-amperes and then decreases thereafter. There are many causes of this phenomenon such as junction temperature [99], Shockley-Read-Hall recombination [100], Auger recombination [97], current leakage [101] and so on. Originally, heat was considered the main reason for the efficiency droop [60]. In 2007, at the meeting of the international conference of nitride semiconductors held in Las Vegas, Lumileds Inc. pointed out that Auger recombination should be blamed for the efficiency droop. Later in 2013, Auger recombination was confirmed as the main cause for the efficiency droop [96].

All the above mentioned recombination mechanisms were taken into consideration in our APSYS simulation which was used in chapter 4 and 5. Based on APSYS simulation, we also discussed the relationship between the radiative recombination rates and the current crowding effect in chapter 5 .

\subsection{Issues and challenges of InGaN/GaN-based LEDs for special}

\section{applications}

With the efficiency of LEDs increasing rapidly, their applications are being intensively studied. Besides general lighting, LEDs also have high potential for applications such as visible light communication, micro-displays, etc. Compared with general lighting, visible light communication and micro-displays need smaller 
sized LEDs with high power density, high current density and high response speed.

However, conventional LEDs cannot satisfy these demands. 


\section{$>$ LEDs used for visible light communication}

With rapidly improving LED technologies, high frequency modulation of LEDs is possible. Due to the aforementioned benefits, the LEDs are deemed suitable for both communication and illumination at the same time, making visible light communication an ubiquitous and economic data transmission solution. Unlike radio waves, visible light is not harmful to the human body. Moreover, the visible light spectrum is not regulated, which opens up a huge new bandwidth for communication. Because of these benefits, visible light communication has been intensively studied in the applications such as in-door communication, car to car communication, traffic lights and street lamps to car communication, and even under water communication. White light LEDs with a yellow phosphor are expected to be the most suitable type of LEDs for visible light communications as they are widely used in the general lighting. However, since the yellow phosphor has a slow response, the bandwidth of the white LEDs can only reach $3 \mathrm{MHz}$ [102]. On the other hand, the blue part of the white light is considered more promising for communication applications. Thus, if an optical blue filter is placed in front of the receiver the bandwidth can reach up to $10 \mathrm{MHz}[102,103]$.

Another way to increase the bandwidth is by the use of RGB white LED, which has a relatively higher response speed. In this way, the bandwidth can be increased to a few tens of $\mathrm{MHz}$ [104]. However, using RGB LEDs for the transmitting information is more expensive and complex compared with white LEDs with a yellow phosphor. Recently, researchers found that micro-LEDs have a huge potential for high data rate transmission. AlInGaN-based resonant-cavity LEDs (RCLEDs) 
provide bandwidth levels around $330 \mathrm{MHz}$ [105], which is significantly higher than white LEDs or RGB LEDs. However, it is a challenge to fabricate RCLEDs [106]. In addition, a transmitter with micro-pixelated LEDs has been proposed [21]. This array of micro-LEDs can provide a bandwidth up to $245 \mathrm{MHz}$ per pixel [21]. Furthermore, it is reported that the bandwidth of the micro-LEDs increases with the enhanced current density and the bandwidth can reach up to $400 \mathrm{MHz}$ [33]. Figure 2.3 shows the relation between the modulation bandwidth and the current density. From Figure 2.3 we can see that the modulation bandwidth is enhanced as the current density is increased. McKendry et al. explained that smaller sized LEDs hold higher maximum modulation bandwidth as the differential carrier lifetime is shorter at high current densities [33]. Therefore, compared with other LED options, LTM-LEDs, which are in micro size (typically smaller than $300 \mu \mathrm{m}$ on one side) and can deliver high current density, are the most promising light sources for the visible light communication, even though the fabrication process may be relatively complex. Nonetheless, for the illumination purposes, the LTM-LEDs should have higher power density to provide enough illumination. In this case, to increase the light response speed and at the same time attain the original illumination function, LTMLEDs need to be further optimized to satisfy the high output power density demand for visible light communications. 


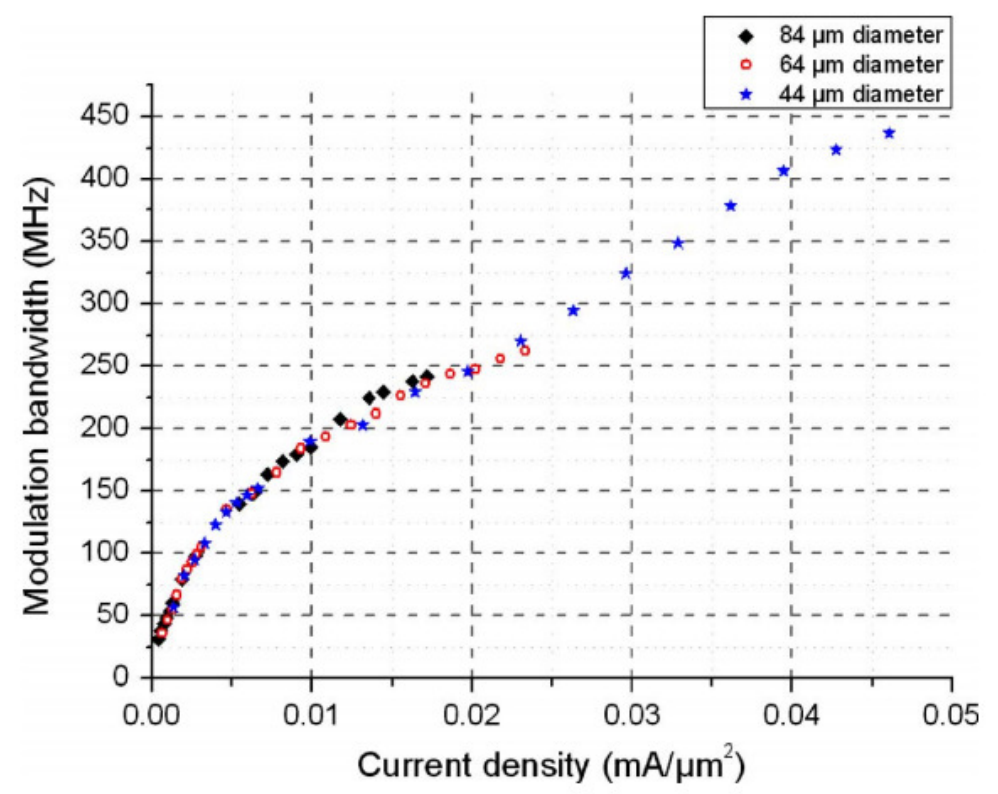

Figure 2.3 Bandwidth versus current density graph for the 450 nm-emitting LEDs [33].

\section{Micro-displays}

InGaN/GaN-based LTM-LED arrays offer a promising technology for selfemissive micro-displays [107]. Micro-displays, which can be used in camcorders, head-mounted displays, pico-projectors, and viewfinders, are very small highresolution displays that can be projected or viewed with the help of mirrors or lenses. To satisfy various high-end applications, micro-displays must have the merits of high resolution, reliability, power efficiency, etc. The R\&D luminous efficacy of GaNbased white LEDs have achieved $300 \mathrm{~lm} / \mathrm{W}$ [2], which is much higher than those of self-emissive electroluminescent emitters and organic LEDs. In addition, the emission intensity of LTM-LEDs can be constant even operated at unusual conditions such as temperatures as high as $120{ }^{\circ} \mathrm{C}$ or as low as $-100{ }^{\circ} \mathrm{C}$, and in high humidity [24]. LTM-LEDs can also provide high color purity. All of these merits 
make LTM-LED arrays an ideal device for micro-display applications. Nevertheless, when micro-displays are used for high-resolution video displays, TVs, and readable displays under sunlight, the output power and efficiency become serious issues [108]. Thus, the output power density of LTM-LEDs needs to be further enhanced. 


\section{Chapter 3. LED wafer growth and fabrication techniques}

All the GaN epitaxial wafers are prepared by metal-organic chemical vapor deposition (MOCVD). Thus, in this chapter, MOCVD, the commonly used method to grow GaN epitaxy, is explained. After that, the fabrication techniques as well as the design and experimental results of flip-chip LEDs and vertical LEDs are introduced.

\subsection{Introduction of MOCVD}

MOCVD is abbreviation for metal-organic chemical vapor deposition system, which is also known as metal-organic vapor phase epitaxy (MOVPE), organometallic chemical vapor deposition (OMCVD) and organometallic vapor phase epitaxy (OMVPE). This is a semiconductor growth technique that can precisely control the thickness of every deposited layer as thin as one nanometer. We can grow many epitaxial layers of different materials to create devices such as LEDs, lasers, photodetectors, solar cells, and so on. To develop those semiconductor epitaxial layers, the chemicals, such as trimethylgallium (TMGa), trimethylindium (TMIn), and trimethylaluminum (TMAl), are vaporized and carried to the reactor by nitrogen or other gases. The chemicals and gases are ultra pure and the dose can be precisely controlled with mass flow controller (MFC). The wafers used in this thesis were grown by MOCVD from Aixtron Inc., which has two main technologies for the 
deposition processes:

\section{$>$ Planetary reactor technology from Aixtron}

The principle of the Planetary Reactor technology is horizontal laminar flow. From Figure 3.1, we can see that there is a gas inlet in the center of the reactor ceiling. To make sure the gases flow homogeneously from the center, over the hot semiconductor substrates to the edge of the chamber, the gas inlet is specially designed and a process pump is used to extract the gases from the edge of the chamber. Each single wafer is placed on a separate disk. To make sure the precursors are uniformly distributed on each individual wafer, the disks are rotating slowly during the deposition process. The precursors will break up and react on the substrates layer by layer. The properties of the epitaxial layer can be modified by changing the amount of gases and the sources of chemicals.

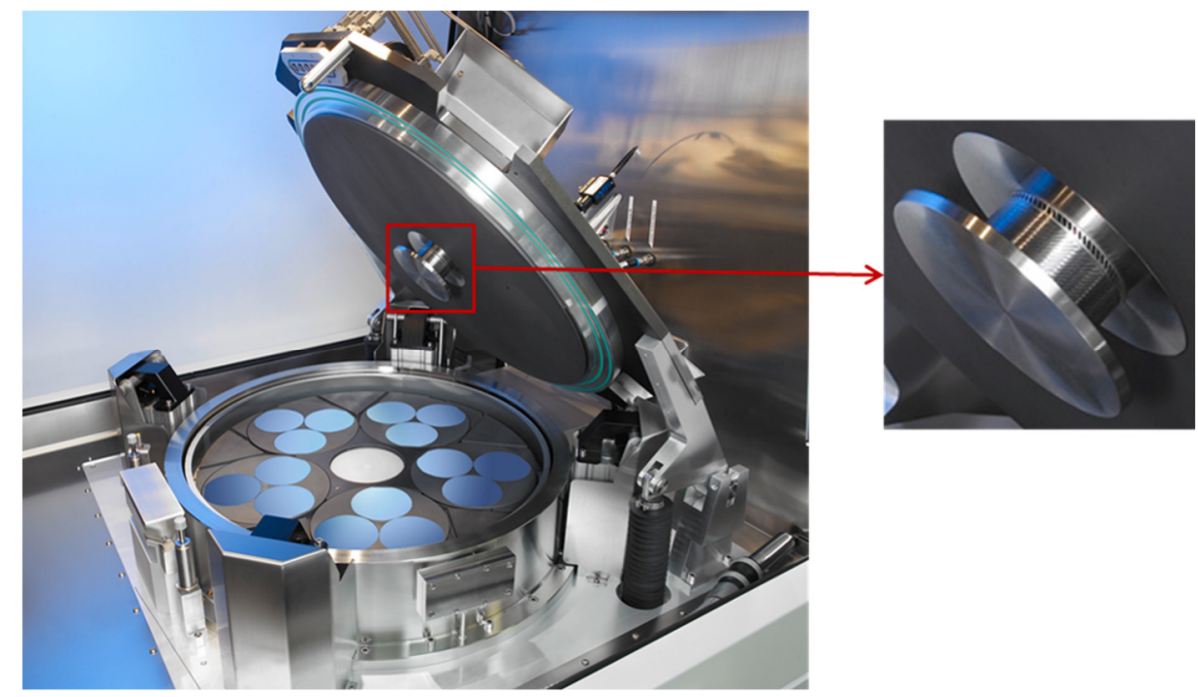

Figure 3.1 The chamber of MOCVD with planetary reactor technology [109]. 


\section{$>$ Close coupled showerhead technology from Aixtron}

The difference of this technology is the gas inlet in the reactor ceiling. From Figure 3.2, we can see that there are a large number of pin holes in the reactor ceiling, which are the gas channels for chemicals coming out to react on the substrates. This design assures that the chemicals mixed in the gases are distributed uniformly throughout all the wafers.

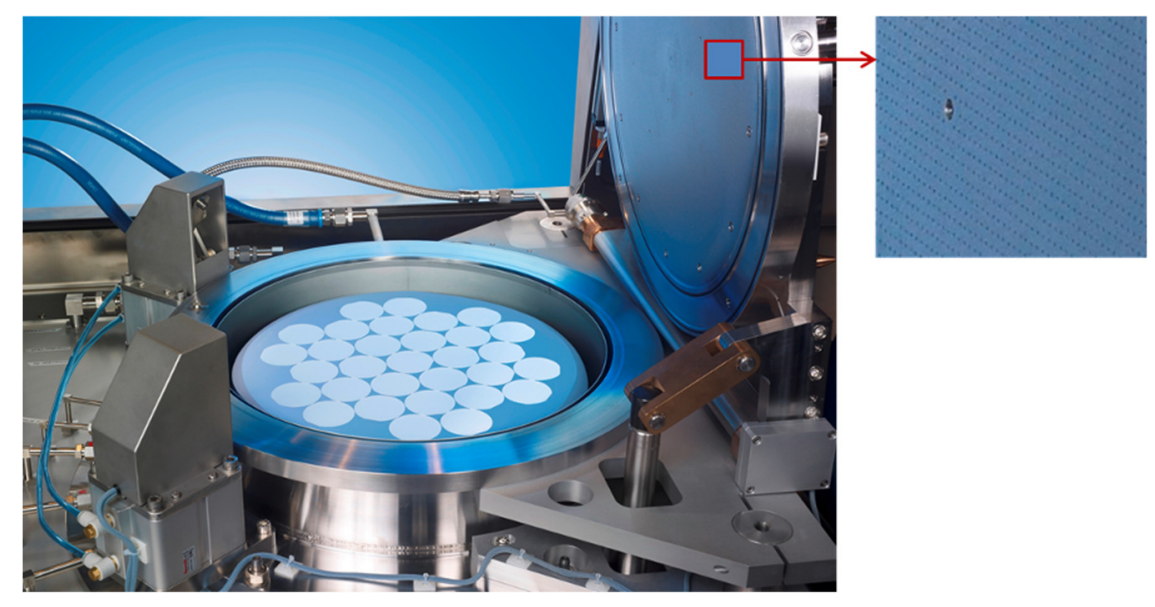

Figure 3.2 The chamber of MOCVD with close coupled showerhead technology [110].

The MOCVD growth of GaN process can be divided into a series of steps, which are shown in Figure 3.3. First, the precursors are transported into the reactor, and then the precursors diffuse or convect through the boundary layer. When the gases mix together, the gas phase reaction starts. Meanwhile, the adsorption of precursors happens and the precursors diffuse on the sapphire substrate or the grown $\mathrm{GaN}$ surface. On the other hand, on the surface, the surface chemical reaction happens and 
the $\mathrm{GaN}$ is deposited. Finally, the precursors, which have not participated in the reaction, and the by-products are desorbed and extracted out of the chamber.

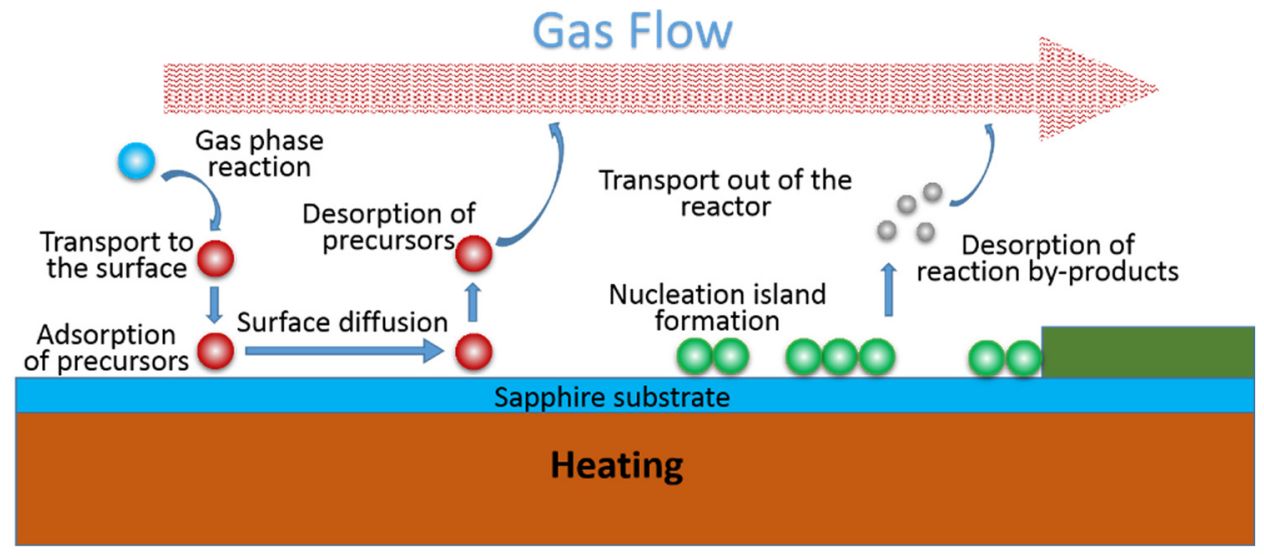

Figure 3.3 Illustration of the MOCVD reaction process.

\subsection{Epitaxial structure of InGaN/GaN-based LEDs}

The LED wafers in this work are grown on c-plane sapphire substrates by Aixtron closed coupled showerhead (CCS) MOCVD system, as shown in Figure 3.4. $\mathrm{Ga}, \mathrm{In}, \mathrm{Al}$, and $\mathrm{N}$ precursors are trimethylgallium (TMGa), trimethylindium (TMIn), trimethylaluminum (TMAl), and ammonia $\left(\mathrm{NH}_{3}\right)$, respectively. A $30 \mathrm{~nm}$ low temperature $\mathrm{GaN}$ buffer layer and $3 \mu \mathrm{m}$ thick unintentionally doped $\mathrm{GaN}$ are first grown, and then followed by a $5.5 \mu \mathrm{m}$ thick n-doped GaN (doping concentration $\left.\approx 5 \times 10^{18} / \mathrm{cm}^{3}\right)$, in which silane $\left(\mathrm{SiH}_{4}\right)$ is used as dopant. Then six (typical design) or eight (new design) pairs of $\operatorname{In}_{0.15} \mathrm{Ga} 0.85 \mathrm{~N} / \mathrm{GaN}(3 \mathrm{~nm} / 12 \mathrm{~nm}$ thick) are grown. Here, one pair of $\operatorname{In}_{0.15} \mathrm{Ga}_{0.85} \mathrm{~N} / \mathrm{GaN}$ means one quantum well consisting of $3 \mathrm{~nm}$ thick $\mathrm{In}_{0.15} \mathrm{Ga}_{0.85} \mathrm{~N}$ well region and $12 \mathrm{~nm}$ thick $\mathrm{GaN}$ barrier region. Next, a 
$20 \mathrm{~nm}$ thick p-doped $\mathrm{Al}_{0.15} \mathrm{Ga}_{0.85} \mathrm{~N}$ electron blocking layer (EBL) is utilized to suppress the excess electron overflow into the $\mathrm{p}-\mathrm{GaN}$ region. Finally a $200 \mathrm{~nm}$ thick p-doped $\mathrm{GaN}$ (hole concentration $\approx 3 \times 10^{17} / \mathrm{cm}^{3}$ ) is grown as the hole transport layer. The p-type conductivity of the EBL and the hole source layer is realized by $\mathrm{Mg}$ doping where Bis(cyclopentadienyl)magnesium $(\mathrm{Cp} 2 \mathrm{Mg})$ is used as the $\mathrm{Mg}$ precursor.

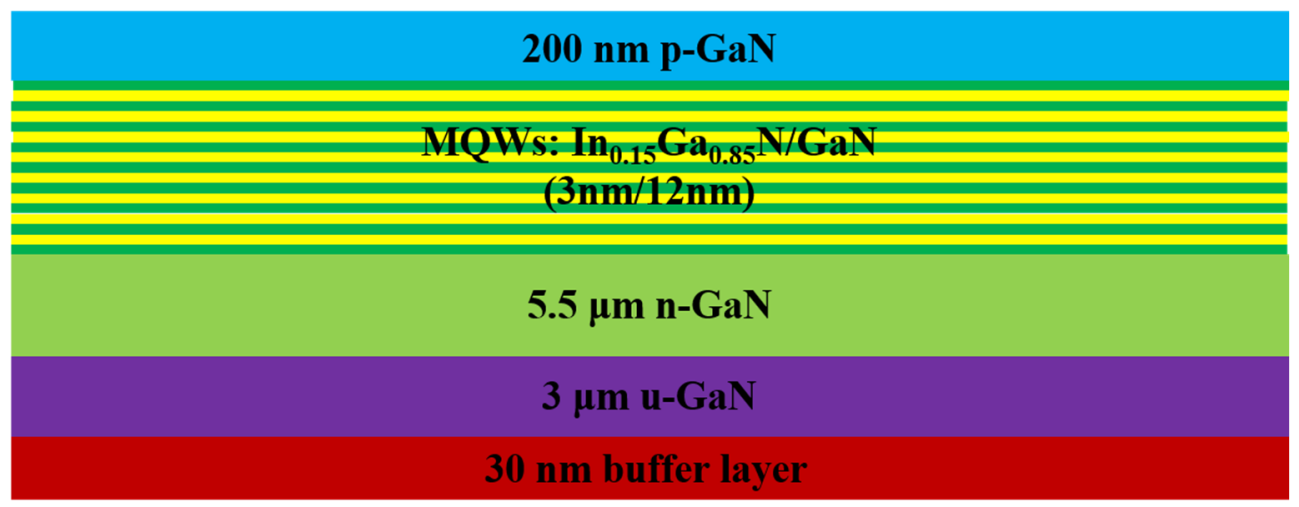

Figure 3.4 Schematic diagram of the InGaN/GaN-based LED wafer.

\subsection{LED fabrication techniques}

After MOCVD growth, wafers need to be further fabricated into LED chips. Since the LED chips are very small and the LED chip structures are complex, photolithography technique is needed to make elements in micro scale. After the photolithography, many other techniques such as reactive-ion etching (RIE), inductively coupled plasmas (ICP), E-beams, Rapid thermal annealing (RTA) are needed to fulfil the LED fabrication process. In this chapter, we will briefly introduce 
the photolithography and other techniques. After that, we show our flip-chip and vertical LEDs and the comparatively studied results.

\subsubsection{Equipment for LED fabrication}

\section{$>$ Photolithography technique}

Photolithography is a process to transfer elements of the circuit in micrometer or even smaller scale from a photomask onto a wafer. The photomask is a transparent quartz plate. On the surface of this plate, patterns are specially designed to satisfy the purpose of making high performance chips. The opaque areas of the photomask are made by covering with nickel. When doing the photolithography, light travels through the transparent area without nickel and the designed pattern will form an image. After exposure, the wafer coated with photoresist, which is a photosensitive material, is developed by a corresponding developer. If the wafer is covered with a positive photoresist, the exposed area dissolves away. Otherwise, the exposed area is remaining. After developing, there is a pattern on the wafer with part of the area covered by photoresist. This patterned wafer is ready for the following procedures, such as RIE or ICP etching, chemical etching, and E-beam deposition. Usually, we need four steps of photolithography for the flip-chip LED fabrication and seven to eleven steps for the vertical LED fabrication. Here, we show the photolithography process with the positive photoresist as an example, as shown in Figure 3.5.

(a)

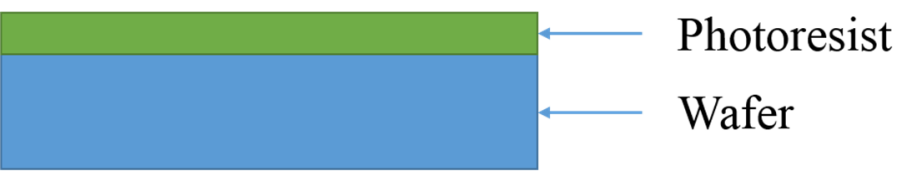


(b)

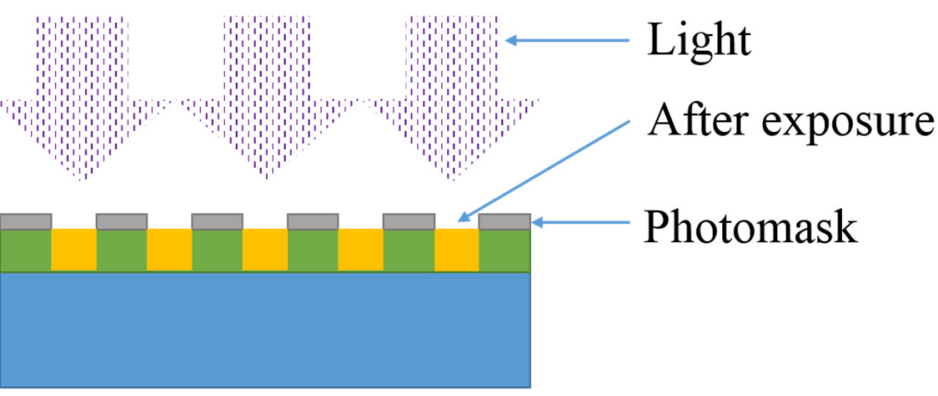

(c)

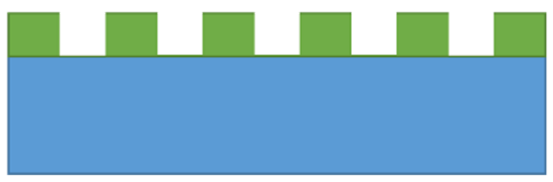

(d)
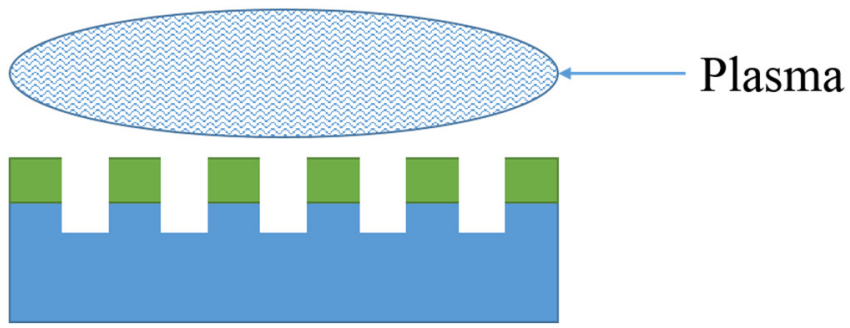

(e)

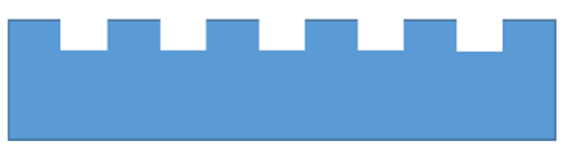

(f)

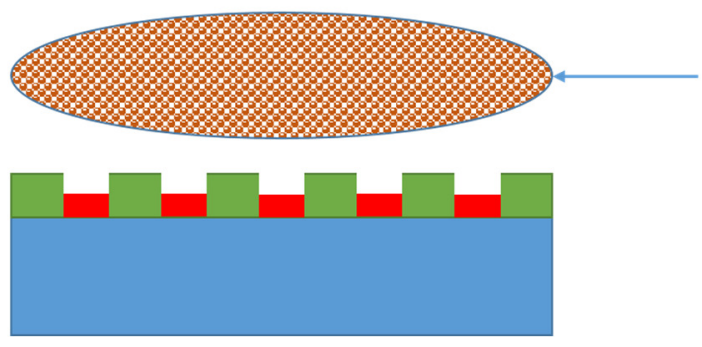

Metal

atoms in

E-beam

chamber

(g)

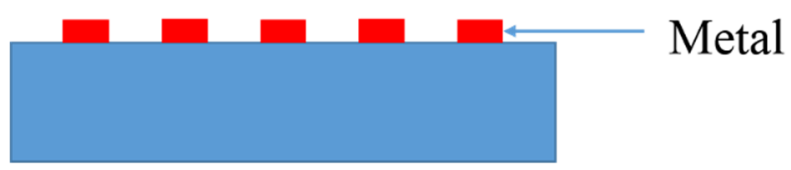

Figure 3.5 The simplified photolithography process: (a) coating photoresist on the wafer surface; (b) exposure to UV light with the 
photomask covering; (c) after developing in the developer. After developing, there are generally two ways to process the wafer, etching and deposition: (d) after step (c) putting the wafer into the dry etching chamber; (e) the etched wafer after removing the photoresist; (f) after step (c) putting the wafer into deposition chamber; (g) the metal pattern on the wafer after removing the photoresist.

\section{Reactive-ion etching (RIE)}

RIE is a dry etching technology commonly used in microfabrication process. RIE uses chemicals as reactants and RF power to generate plasma to remove the atoms on the wafers. There are generally two processes in the RIE chamber, highenergy ions physically hitting the wafer surface and reacting chemically with the materials. As most of the reactive ions are vertically delivered in the chamber, very anisotropic etch profiles can be produced by RIE dry etching. The etching conditions in the system rely strongly on the chosen parameters, such as RF power, the pressure in the chamber, and the gas flows. To get the ideal design profiles we should choose and control the proper parameters. In our LED fabrication process, RIE is mainly used for etching $\mathrm{SiO}_{2}, \mathrm{GaN}$, and W.

\section{$>$ Plasma-enhanced chemical vapor deposition (PECVD)}

PECVD is a technique widely used to grow thin films on a substrate. In our LED fabrication process, PECVD is generally aimed to deposit $\mathrm{SiO}_{2}$ films. Compared with common CVD, PECVD uses plasma to assist the chemical reactions in the process. The plasma is generated by the RF power or DC discharge. The two 
electrodes of the power are separated, and the reacting gases are filled-in between the electrodes.

\section{Electron beam physical vapor deposition (E-beam)}

E-beam is a system that uses electron beam to bombard the target anode material, generating gaseous phase atoms and coating the wafer in the chamber with a uniform thickness of the target anode material. The electron beam is generated by a charged $\mathrm{W}$ filament in the high vacuum chamber, and then the electron beam is controlled by the magnetic field impinging on the target anode material. In our LED fabrication process, E-beam is mainly used to deposit nickel, silver, titanium, and gold.

\section{Sputter deposition}

Sputter deposition is a technique to grow thin film onto a substrate such as a gallium nitride wafer by sputtering. It is a physical vapor deposition method. Sputtering is the emission of the target material by ion or atom bombardment. After sputtering, some of the sputtered atoms are ejected from the target material onto the substrate. During the sputtering process, the sputtering gas, which is generally an inert gas, is used to bombard the target material. The sputtering gas is always carefully chosen to transfer the momentum efficiently. The rule is that the atomic weight of the target material should be similar with that of the sputtering gas.

Compared with evaporation, the sputter deposition can sputter high melting point materials. Furthermore, the deposited films have nearly the same composition as the source material. Typically, the sputtered films have stronger adhesion on the 
substrate than the evaporated films. However, the shortcoming is that it is hard to do the lift off process.

\section{Rapid thermal annealing (RTA)}

RTA is a commonly used semiconductor device manufacturing process. This process heats the wafers to a high temperature in a short time which is around a few seconds or shorter; then maintains this high temperature for a short time; and finally cools the wafer naturally. The cooling process must be slow in order to prevent dislocations. The rapid heating rates are generally realized by lasers or high intensity lamps. For different effects, special heat treatment needs to be designed. The RTA treatment can be used to activate dopants, change the interfaces of films, repair damages, move dopants, and change states of grown films. In our LED fabrication process, RTA is used for annealing the mirror to get the highest reflection and lowest ohmic contact resistance.

\subsection{Flip-chip and vertical LEDs}

After MOCVD growth, wafers are fabricated into different designed chips. Here, we introduce high-power LEDs with the size of $1 \times 1 \mathrm{~mm}^{2}$ used for illumination. Two kinds of broad area LEDs will be introduced in this section: flip-chip and vertical LEDs. For each kind of LEDs, they both have their own special design to explore the optimal performance with respect to fabrication. In our structure, we deposit a $250 \mathrm{~nm}$ silver-based metal layer $(\mathrm{Ni} / \mathrm{Ag} / \mathrm{Ni} / \mathrm{Au})$ on the $\mathrm{p}-\mathrm{GaN}$ as the current 
spreading layer and this layer also has strong ability to reflect light, so we named this layer "mirror". This mirror layer is used for both types of LEDs.

For this part, we did these experiments in 2013. The LEDs were fabricated using our mature fabrication process and the values of the bias voltage and output power were similar with those from the best companies in the same year. Hence even one percent decrease in the bias voltage or increase in the output power needed a large amount of hard work and the improvement was not significant. The current-voltage characteristics of the LED chips were measured using a LED quick tester (M2442S9A Quatek Group) and the optical output power was measured by the integrating sphere attached to an Ocean Optics spectrometer (QE65000), which was calibrated with a standard light source. The measurement accuracy for voltage is $0.001 \mathrm{~V}$; the measurement accuracy for output power is $0.01 \mathrm{~mW}$. The devices were on the same wafer for different types, and the data on the same wafer was tested on the same day by the same person using the same testing equipment. For each type, at least 20 LED devices were tested. To confirm the improvement, experiments were repeated at least 4 times. For the bias voltage, the difference under $0.01 \mathrm{~V}$ may due to the testing error and $0.02 \mathrm{~V}$ difference can be recognized as reliable. For the power, the difference under $0.5 \%$ may be due to the testing error and $0.5-1 \%$ difference can be recognized as reliable.

For the lumens, we obtained the value through the standard method in the industry as follows: Firstly, we acquire commercial LEDs having the same size and wavelength with our LED devices as reference. The commercial LEDs have lumen data from the company. Secondly, the phosphor on the commercial LEDs is removed. 
Thirdly, the output power in $\mathrm{mW}$ is tested both for the commercial LEDs and our LED devices by the same person on the same day using the same testing equipment. After testing, the commercial LED and our LED device with the same wavelength are matched together as one pair. Fourthly, the lumens of our LED device are calculated with the standard method in the industry. For example, for a pair of LEDs with the same wavelength, the commercial LED is $n_{l} \mathrm{~lm}$, which is obtained from the company, and the output power tested by us is $m_{1} \mathrm{~mW}$; the tested output power of our LED device is $m_{2} \mathrm{~mW}$. Then lumens of our LED device are $n_{2}=m_{2} \times n_{1} / m_{1}$. For the lumen/watt, as the voltage and current are tested, the electrical power can be calculated in watt. Then the lumen/watt can be obtained.

\subsubsection{Flip-chip LEDs}

\section{$>$ The design of flip-chip LEDs}

Figure 3.6 illustrates the diagram of a flip-chip LED. To fabricate the flip-chip LEDs, the mesa is first etched down to the $\mathrm{n}-\mathrm{GaN}$ by RIE. Then the mirror layer is deposited, which covers the top of the mesa with the marginal area of the edge uncovered. Before depositing p- and n-pad, an insulated layer is deposited and the pad area is etched. The main fabrication process is shown in Figure 3.7. 


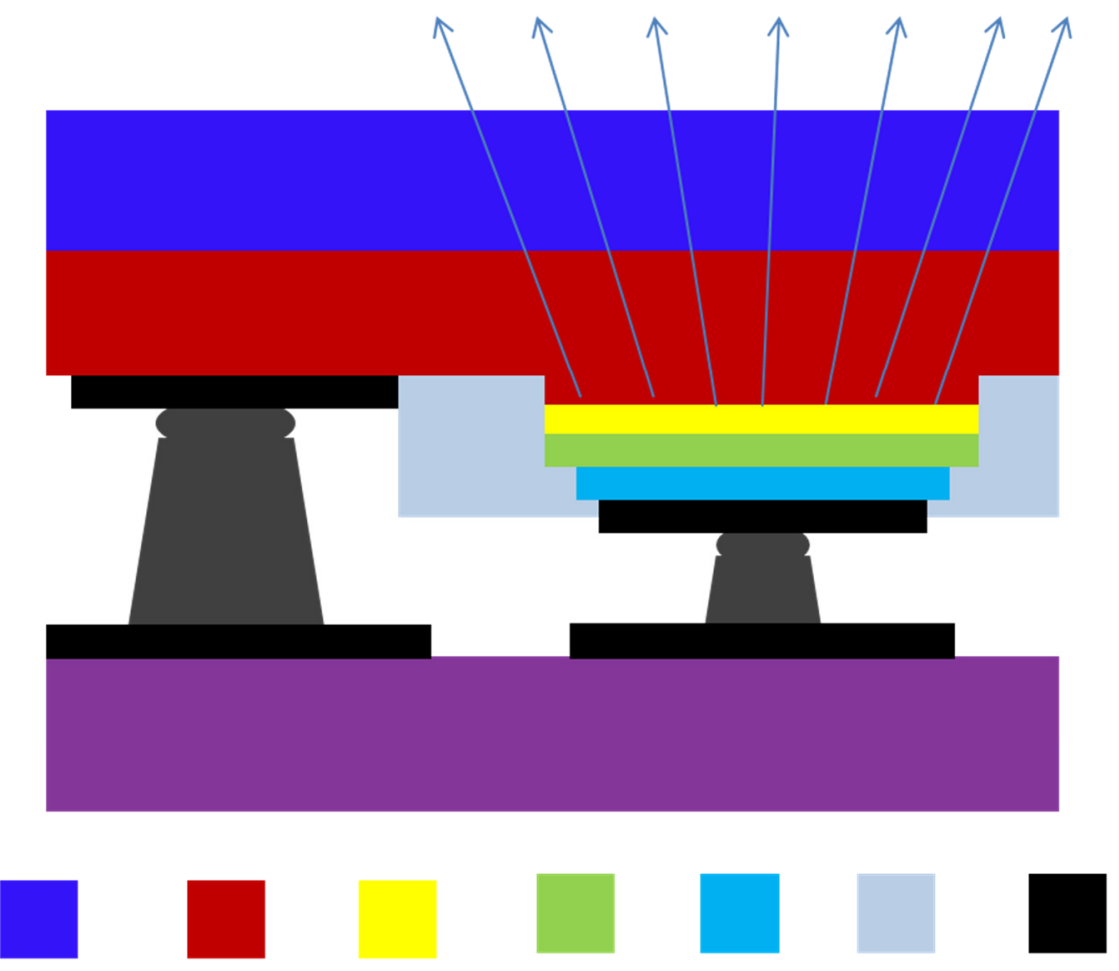

Sapphire n-GaN MQW p-GaN Mirror $\mathrm{SiO}_{2}$ p-\&n-Pad

Figure 3.6 The diagram of flip-chip LEDs. 

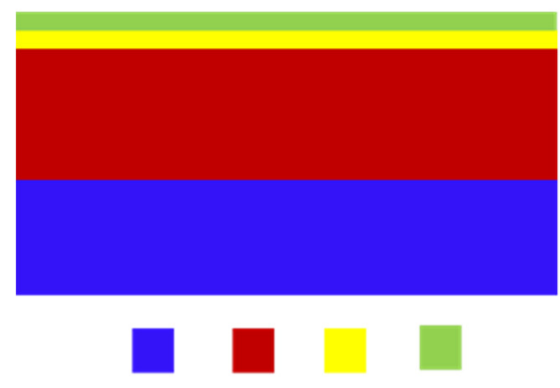

Sapphire n-GaN MQW p-GaN

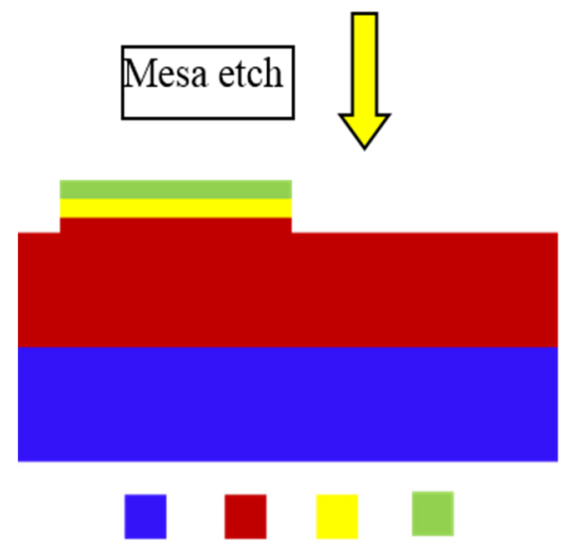

Sapphire n-GaN MQW p-GaN

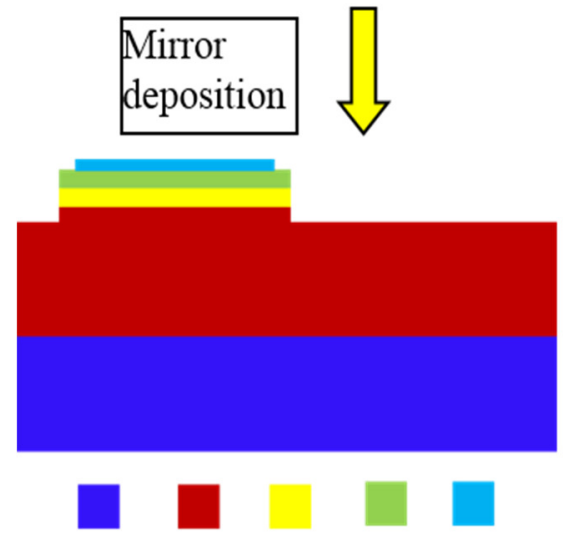

Sapphire n-GaN MQW p-GaN Mirror
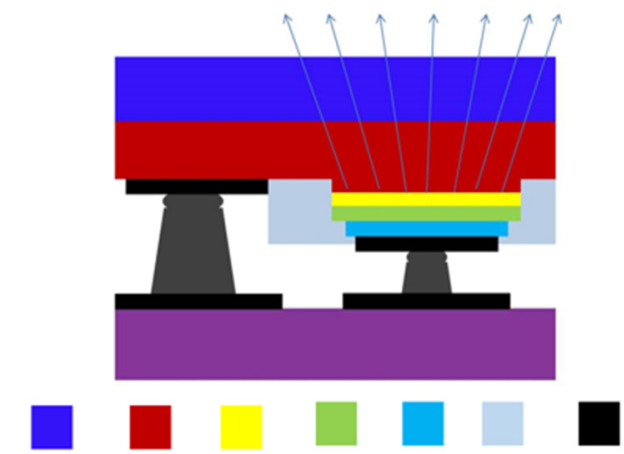

Sapphire n-GaN MQW p-GaN Mirror $\mathrm{SiO}_{2}$ p-\&n-Pad

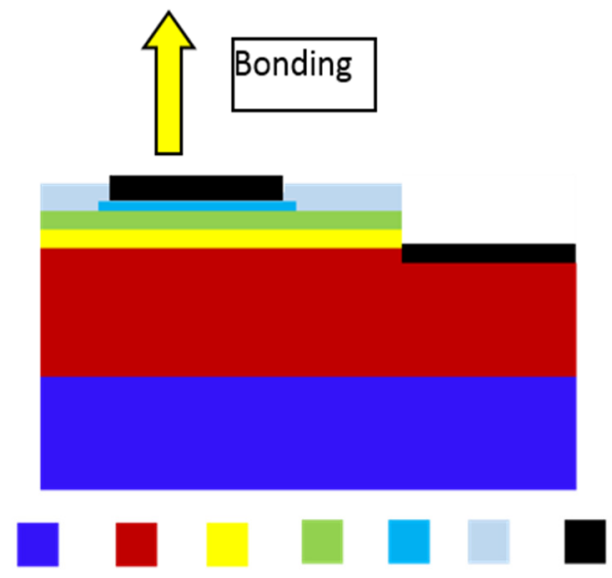

Sapphire n-GaN MQW p-GaN Mirror $\mathrm{SiO}_{2}$ p-\&n-Pad
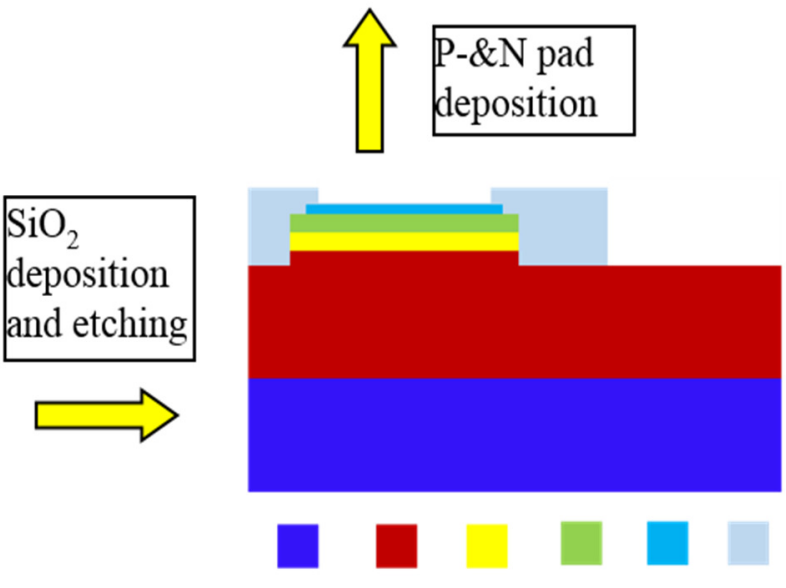

Sapphire n-GaN MQW p-GaN Mirror $\mathrm{SiO}_{2}$

Figure 3.7 Flip-chip LED fabrication process.

We designed two types of flip-chip LEDs with the same area and fabricated on the same wafer. For both types, the p-pad connects to the mirror, which is a current 
spreading layer for $\mathrm{p}-\mathrm{GaN}$, through $\mathrm{p}$-Via and the $\mathrm{n}$-pad connects to the $\mathrm{n}-\mathrm{GaN}$ through n-Via, as can be seen in Figure 3.8. From Figure 3.8(a), for Type 1, we can see that both the p-pad and n-pad have a comb structure. This design takes the higher p-GaN resistance into consideration and thus makes the p-pad larger. The benefit of Type 1 is that the current spreading for the p-pad is more uniform and the p-GaN current spreading relies less on the mirror. Type 2 has a simpler design compared to Type 1 as shown in Figure 3.8(b). This design takes the low resistance mirror into account and the p-GaN current spreading relies more on the mirror. As the p-pad connects mirror through $\mathrm{p}-\mathrm{Via}$ and the mirror is covered onto the $\mathrm{p}-\mathrm{GaN}$ mesa, if the conductivity of the mirror is good enough, the p-GaN current spreading is guaranteed, or if the conductivity of the mirror is insufficient, the p-GaN current will be crowded under the p-pad and the efficiency will deteriorate.
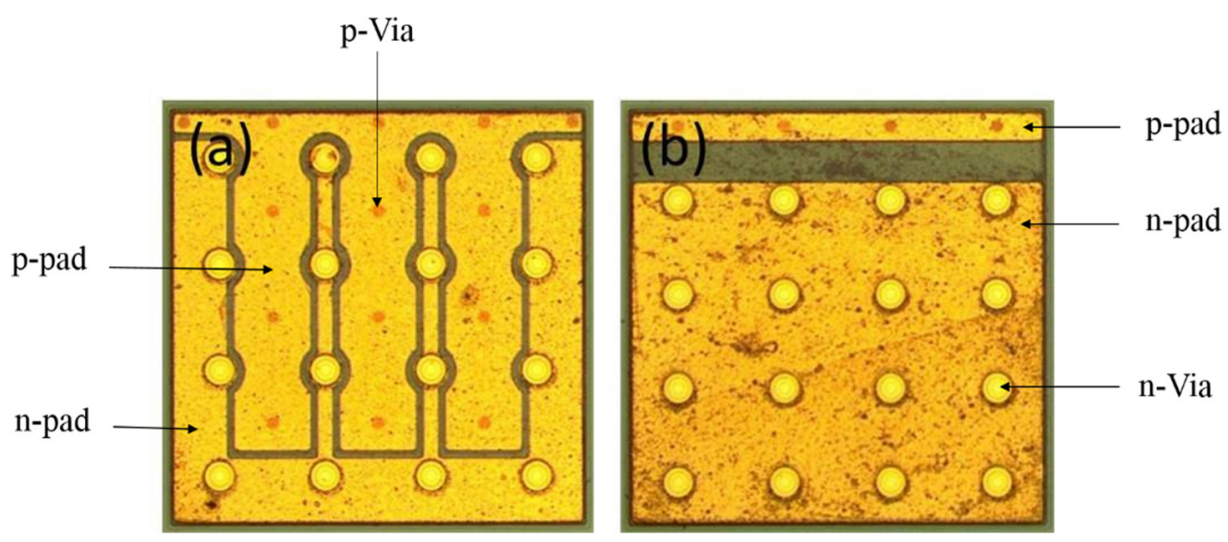

Figure 3.8 Flip-chip LEDs with the same area: (a) Type 1 and (b)

Type 2.

\section{$>$ Experimental results}


The experimental results of broad area flip-chip LEDs are summarized in Table 3.1. From the table, we can see that Type 1 and Type 2 have similar performance. At the same current of $20 \mathrm{~mA}$ or $350 \mathrm{~mA}$, Type 1 and Type 2 have similar bias voltage. This demonstrates that the mirror performs well in current spreading for $\mathrm{p}-\mathrm{GaN}$. This is significant for the future flip-chip LED design. As for the light output power, Type 1 and Type 2 have similar performance. This can be deduced from the similar n-Vias distribution on both Type 1 and Type 2, which contributes to similar current spreading. In conclusion, Type 1 and Type 2 have similar efficiency, and the mirror is effective in the current spreading for $\mathrm{p}-\mathrm{GaN}$.

Table 3.1 The experimental results of the bias voltage and power of flip-chip LEDs with the same area.

\begin{tabular}{|c|c|c|c|c|c|}
\hline \multirow{2}{*}{ Type } & \multicolumn{2}{|c|}{ Bias voltage } & \multicolumn{3}{c|}{ Power } \\
\cline { 2 - 6 } & $20 \mathrm{~mA}$ & $350 \mathrm{~mA}$ & \multicolumn{3}{|c|}{$350 \mathrm{~mA}$} \\
\hline Type 1 & $2.69 \mathrm{~V}$ & $3.29 \mathrm{~V}$ & $245 \mathrm{~mW}$ & $149 \mathrm{~lm}$ & $129 \mathrm{~lm} / \mathrm{W}$ \\
\hline Type 2 & $2.67 \mathrm{~V}$ & $3.26 \mathrm{~V}$ & $246 \mathrm{~mW}$ & $150 \mathrm{~lm}$ & $131 \mathrm{~lm} / \mathrm{W}$ \\
\hline
\end{tabular}

\subsubsection{Vertical LEDs}

\section{$>$ The design of vertical LEDs}

Figure 3.9 illustrates the simplified diagram of vertical LEDs. The advantage of vertical LEDs is that it has excellent current spreading ability and heat diffusion ability. As the whole $\mathrm{p}-\mathrm{GaN}$ is covered with metal, it has very good current spreading for $\mathrm{p}-\mathrm{GaN}$ and copper conducts heat very well. 


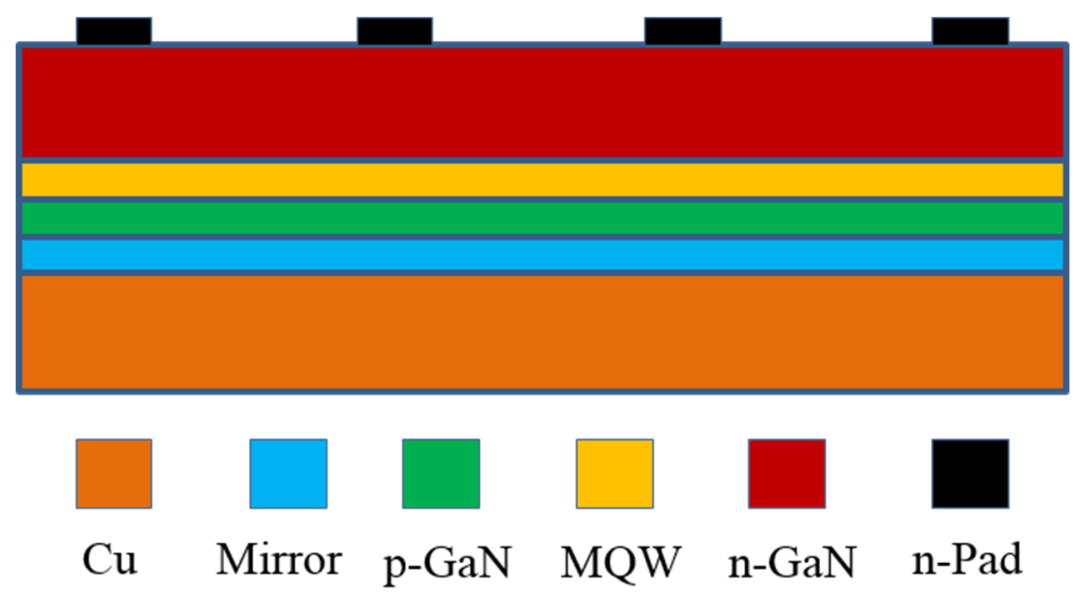

Figure 3.9 The schematic diagram of vertical LEDs.

To enhance the performance of vertical LEDs, four different types of n-electrode layout are designed and fabricated on the same wafer with the same area, as shown in Figure 3.10. Type 1 has broader pad wires, which can benefit the current spreading in the wires and decrease the bias voltage at a certain current, as shown in Figure 3.10(a). However, it sacrifices the active light emission area, which affects the total output power to some extent. Type 2 has similar design as Type 1 with narrower pad wires, which can be seen from Figure 3.10(b). This design takes more active light emission area into consideration, which aims to improve the total output power. Of course, this narrower pad wires will sacrifice the bias voltage at a given current. Type 3 includes a new design of n-pad, and has the same pad wire width and effective pad wire length with Type 2. From Figure 3.10(c) we can see that the LED mesa is surrounded by electrode wire and on the mesa there are three intertwined fingers. Compared with Type 2, this design saves some of the active light emission area as the surrounding electrode wire does not occupy any mesa area. However, as the wires are sparse on the mesa, the current uniformity on the mesa at relatively high current 
will be sacrificed. Compared with Type 3, Type 4 has a current blocking layer under the electrode fingers. This design can prevent the current from flowing perpendicularly from the n-electrode fingers toward the bottom p-electrode. Thus, it will significantly improve the lateral current spreading, which is good for the current uniformity. On the other hand, as the current is blocked under the electrode, the bias voltage of this Type 4 is increased to some extent.
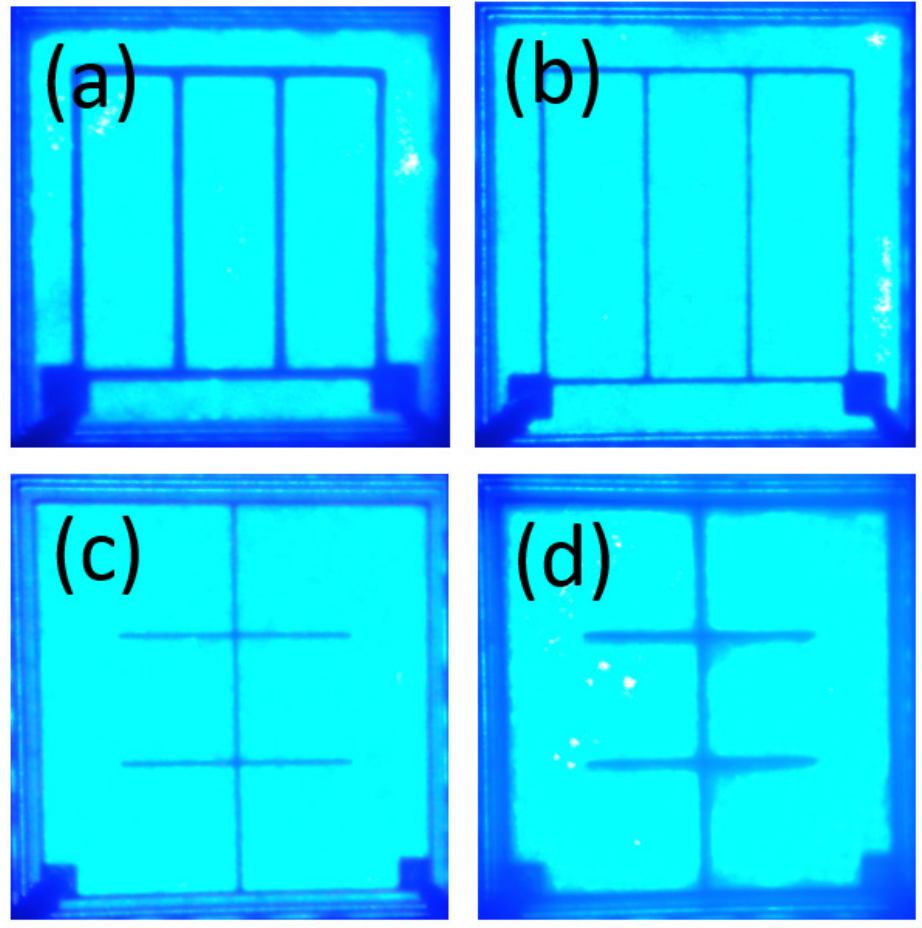

Figure 3.10 Vertical LEDs with the same area lighted up at $1 \mathrm{~mA}$ : (a)

Type 1(T1); (b) Type 2 (T2); (c) Type 3 (T3); and (d) Type 4 (T4).

\section{Experimental results}

Table 3.2 summarizes the measured current versus bias voltage and light output power versus current results of the vertical LEDs. From Table 3.2 we can see that at the same current, Type 1 has the lowest bias voltage, but also the lowest power as a 
whole; Type 4 has the highest bias voltage, but the highest power; types 2 and 3 have performance in between Type 1 and Type 4 .

Through calculation, we find that Type 2 has an $11 \%$ increase for the output power than Type 1 at $350 \mathrm{~mA}$. This increase can be attributed to the extra active light emission area obtained by decreasing the width of the wires. With a larger active light emission area, the light extraction efficiency is higher as more light can come out from the LED device without being absorbed and reflected by the n-electrode metal fingers. On the other hand, there is also a $4 \%$ increase in the bias voltage. The underlying reason is that the resistance of the n-electrode wires increase because of the narrower width. Hence, we can conclude that if we increase the width of the npad, the bias voltage will decrease but the light output power will decrease.

Type 3 has slightly higher bias voltage compared with Type 2 . This is because the wires are sparse on the mesa in Type 3 and hence the current spreading is relied on the $\mathrm{n}-\mathrm{GaN}$. As is well-known, $\mathrm{n}-\mathrm{GaN}$ has much higher resistance than the metal electrode. Therefore, the bias voltage of Type 3 is higher than that of Type 2 in a certain current. On the other hand, for the light output power, Type 2 has similar output power with Type 3 at $350 \mathrm{~mA}$, and slightly higher at $700 \mathrm{~mA}$, but when the current gets down to $20 \mathrm{~mA}$, the light power is lower than Type 3. The better output power of Type 3 at low current can be attributed to the larger active light emission area, which has a smaller electrode area on the mesa. However, this decreases the current uniformity at high current, which is the reason for higher efficiency droop.

Compared with Type 3, we can see that Type 4 has somewhat increasing in the bias voltage under the same current and has significant improvement in the output 
power. We find that the output power of Type 4 has a $17 \%$ improvement compared to Type 3 at $350 \mathrm{~mA}$. This improvement only increase the bias voltage by $1.5 \%$. This is because with the current blocking layer under the electrode, the current paths will travel in a lateral way instead of directly going to the p-electrode in a perpendicular way. Hence, the current uniformity on the mesa is much better than Type 3 and the resistance as a whole also increase because of the inserting current blocking layer under the electrode fingers. Therefore, it is necessary to adopt a current blocking layer for the enhancement of the vertical LEDs performance. In summary, compared with the increase of light power, the increase of bias voltage is much smaller.

Table 3.2 The experimental results of bias voltage and power of four type vertical LEDs with the same area.

\begin{tabular}{|c|c|c|c|c|c|c|}
\hline \multirow{2}{*}{ Type } & \multicolumn{3}{|c|}{ Bias voltage Measurement (V) } & \multicolumn{3}{c|}{ Power Measurement (mW) } \\
\cline { 2 - 7 } & $20 \mathrm{~mA}$ & $350 \mathrm{~mA}$ & $700 \mathrm{~mA}$ & $20 \mathrm{~mA}$ & $350 \mathrm{~mA}$ & $700 \mathrm{~mA}$ \\
\hline $\mathrm{T} 1$ & 2.78 & 3.17 & 3.64 & 9.80 & 151 & 280 \\
\hline $\mathrm{T} 2$ & 2.78 & 3.30 & 3.67 & 9.70 & 167 & 310 \\
\hline $\mathrm{T} 3$ & 2.80 & 3.33 & 3.69 & 10.50 & 166 & 306 \\
\hline T4 & 2.80 & 3.38 & 3.79 & 12.60 & 194 & 361 \\
\hline
\end{tabular}

Note: For the vertical LEDs, $194 \mathrm{~mW}$ corresponds to about $120 \mathrm{~lm} / \mathrm{W}$.

\subsection{Conclusions}

In conclusion, the equipment for LED fabrication was introduced. Both flipchip and vertical LEDs were comparatively studied, respectively. Both electrical and optical performance is carefully studied experimentally. For the flip-chip LEDs, it is 
demonstrated that the $250 \mathrm{~nm}$ silver-based mirror performs very well in the $\mathrm{p}$-GaN current spreading. This means that to reduce the n-electrode resistance, the n-pad area can be designed as large as possible. In other words, small n-electrode resistance can benefit the performance of flip-chip LEDs. For the vertical LEDs, it is suggested that the active light emission area and the current uniformity are two important factors for high output power. Therefore, the optical performance can be enhanced by inserting a current blocking layer between the $\mathrm{n}$-finger and the $\mathrm{n}-\mathrm{GaN}$ to improve the uniformity of current spreading.

This part of work provides a good rule of thumb in the following LTM-LED fabrication: in Chapter 4 and Chapter 5, the LTM-LEDs in the flip-chip configuration are fabricated using the same processes as the flip-chip LEDs; in chapter 7, we propose a vertical LTM-LEDs to enhance the response speed for the application of visible light communication. 


\section{Chapter 4. Low thermal-mass LEDs: size effect and limits}

With the luminous efficacy rapidly increasing and unique applications of LEDs being intensively studied, the size of LEDs has a trend of becoming smaller. Hence, based on the fabrication techniques introduced in Chapter 3, we further fabricated the LTM-LEDs. In this chapter, LTM-LEDs were developed and demonstrated in the flip-chip configuration, studying both experimentally and theoretically the enhanced electrical and optical characteristics and the limits. LTM-LED chips in $25 \times 25 \mu \mathrm{m}^{2}, 50 \times 50 \mu \mathrm{m}^{2}, 100 \times 100 \mu \mathrm{m}^{2}$ and $200 \times 200 \mu \mathrm{m}^{2}$ mesa sizes were fabricated and comparatively investigated. Here it was revealed that both the electrical and optical properties are improved by the decreasing chip size due to the reduced thermal mass. With a smaller chip size (from $200 \mu \mathrm{m}$ to $50 \mu \mathrm{m}$ ), the device generally presents higher current density against the bias and higher power density against the current density. However, the $25 \times 25 \mu \mathrm{m}^{2}$ device behaves differently, limited by the fabrication margin limit of $10 \mu \mathrm{m}$. The underlying mechanisms of these observations are uncovered, and furthermore, based on the device model, it is proven that for a specific flip-chip fabrication process, the ideal size for LTM-LEDs with optimal power density performance can be identified. This chapter is partially based on "Low thermal-mass LEDs: size effect and limits" published in Optics Express, Vol. 22 No. 26. 


\subsection{Introduction}

As mentioned previously, InGaN/GaN-based LEDs are playing an important role in many fields including artificial lighting [1, 40-42, 111, 112], displays [41, 43, 113], communications [21],[33], and neurosciences [44]. To fulfill the requirements of these different applications, LEDs always have been designed into various sizes and geometries. As widely accepted, one of the important applications for GaN based LEDs is general lighting. In this respect, broad-area LEDs have usually been commonly adopted to meet the requirements of high output power, high efficiency and reliability $[55,56,59]$. The current density of the broad-area LEDs is typically in the range of $1 \mathrm{~A} / \mathrm{cm}^{2}$ to $100 \mathrm{~A} / \mathrm{cm}^{2}$. Yet, such LEDs with much larger chip sizes are not effective in achieving higher light extraction efficiency [28],[29], higher power densities [30],[31], and faster pulsed operation [29] even when operating at higher current densities, which make them unsuitable for the applications that require high current densities, high power densities, and high-speed operation. Therefore, smaller sized LEDs have been proposed, which are deemed more appropriate for such applications including micro-displays [32] and visible communications [33], which typically need the LEDs to operate in the current density range beyond 1,000 $\mathrm{A} / \mathrm{cm}^{2}$. To understand why smaller-sized LEDs can sustain higher current densities and deliver higher power densities, LEDs of different sizes were previously reported [34].

In the fabricating process of both conventional and micro-sized LEDs, it is important to prevent surface recombination on the mesa sidewalls, and the current spreading layer should therefore be made smaller than the p-GaN mesa 
[29],[30],[114]. Nominally, p-contact area is used as the LED effective area to calculate the current density and power density. However, it is reported that the current will not stop at the edge of the spreading layer, and instead will decrease exponentially away from the edge of the p-contact [30]. As a result, the mesa area around the p-contacts, which is not covered by the metal-contact, will also emit light, even though not as strong as the covered area. Therefore, there exists a deviation from the effective area. This deviation will become even larger when the size of the LEDs decreases to several tens of micrometers. On the other hand, as a conductive path, the distance between the n-contact and the LED mesa has a substantial influence on the current spreading and output power of the LEDs, especially for the smaller sized LEDs [30]. This will place a constraint limiting the potential improvement achievable with the size effect. However, previous works did not study these issues or report the limits.

In this work, we developed and comparatively studied LTM-LEDs with their mesa area varied from $200 \times 200 \mu \mathrm{m}^{2}$ down to $25 \times 25 \mu \mathrm{m}^{2}$ in the flip-chip configuration. To make a fair comparison between the different sizes of the LEDs, we purposely keep the distance between the n-pad and the LED mesa a constant. Also, we take the mesa area (instead of the p-contact area) as the effective area for the calculation of the current density and power density, which is more meaningful and accurate for the assessment of the smaller sized LEDs. The revaluation of the size-dependent electrical and optical properties of the LTM-LEDs is carefully studied here, which is found to help to identify the optimized size of the LTM-LEDs for the high-power density and for high-speed applications. 


\subsection{Growth, fabrication and simulation of different sized LTM- LEDs}

The LED epitaxial wafers used in this work were grown on c-plane sapphire substrates by the MOCVD system. The wafer we used has 6 quantum wells, and the other details of the growth process can be found in "3.2. Epitaxial structure of InGaN/GaN-based LEDs" in Chapter 3.

Following the MOCVD growth, the LED wafers were fabricated into flip-chip LED devices using standard micro fabrication techniques, as shown in Figure 4.1. Mesa areas in four different sizes of $25 \times 25 \mu \mathrm{m}^{2}, 50 \times 50 \mu \mathrm{m}^{2}, 100 \times 100 \mu \mathrm{m}^{2}$ and $200 \times 200 \mu \mathrm{m}^{2}$ were prepared using reactive ion etching (RIE) on the same wafer. $\mathrm{Ni} /$ Ag-based metal layers were deposited and annealed in oxygen ambient as the current spreading layer. The areas of the current spreading layers are $15 \times 15 \mu \mathrm{m}^{2}$, $40 \times 40 \mu \mathrm{m}^{2}, 90 \times 90 \mu \mathrm{m}^{2}$ and $190 \times 190 \mu \mathrm{m}^{2}$, correspondingly. Ti/Au $(30 \mathrm{~nm} / 1,000 \mathrm{~nm}$ thick) metal layers were deposited by an e-beam evaporation as the p- and n-contact pads. The current-voltage characteristics of the LED chips were measured using a LED tester (M2442S-9A Quatek Group) and the optical output power was measured by an integrating sphere attached to an Ocean Optics spectrometer (QE65000). 


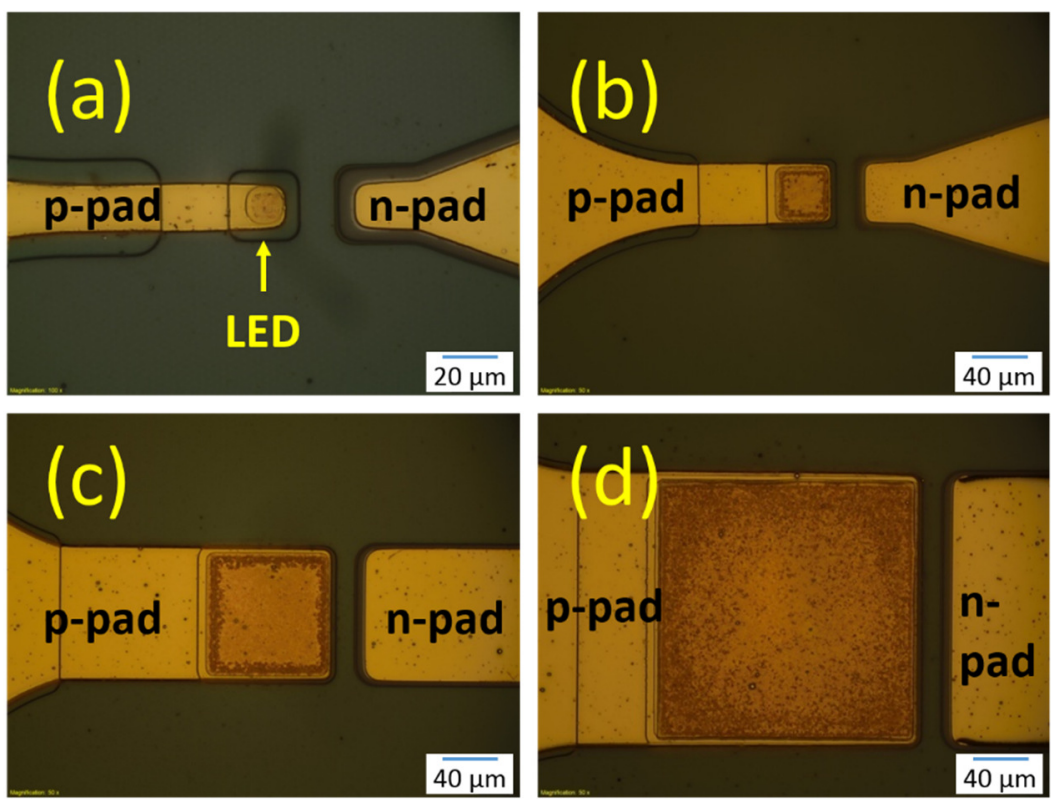

Figure 4.1 The microscope images of LTM-LEDs with different sizes:

top-view images for (a) $25 \times 25 \mu \mathrm{m}^{2}$, (b) $50 \times 50 \mu \mathrm{m}^{2}$, (c) $100 \times 100$ $\mu \mathrm{m}^{2}$, and (d) $200 \times 200 \mu \mathrm{m}^{2}$.

To understand the underlying physics, numerical modelling was performed to reveal the effect of the mesa size on the electrical and optical characteristics. In our simulations, we used the APSYS software to self-consistently solve the Poisson equation, the continuity equation, and Schrödinger equation with proper boundary conditions. The carrier screening effect in the InGaN quantum wells was taken into account using the self-consistent six-band $k \bullet p$ theory [115]. The Auger recombination coefficient was set to be $1 \times 10^{-30} \mathrm{~cm}^{6} \mathrm{~s}^{-1}[116]$. The Shockley-ReadHall (SRH) lifetime was set to be 43 ns [116]. Simultaneously, due to the crystal relaxation through dislocation generation during the growth, a $40 \%$ of the theoretical polarization induced sheet charge density was assumed in the heterojunction [114]. In the $\mathrm{In}_{0.15} \mathrm{Ga}_{0.85} \mathrm{~N}$ quantum well regions, the energy band offset ratio $\Delta \mathrm{E}_{\mathrm{C}} / \Delta \mathrm{E}_{\mathrm{V}}=$ 
70/30 [117] was taken. The other parameters used in the simulation can be found elsewhere [118],[58].

\subsection{Electrical and optical performances of LTM-LEDs and discussion}

Figures 4.2(a) and 4.2(b) are the experimental measurement and numerical simulation of the current density-voltage $(J-V)$ characteristics for the LTM-LEDs with different sizes. The computed results show the same trend as the experimental data for different sized LEDs. From Figures 4.2(a) and 4.2(b), it is clear that the $J$-V property is improved as the device size decreases from $200 \times 200 \mu \mathrm{m}^{2}$ to $50 \times 50 \mu \mathrm{m}^{2}$. Higher current density can be obtained in smaller devices at a given bias voltage. Although the simulation results are consistent with the experimental results, the physical reason of this phenomenon is not straightforward to understand. Therefore, we further developed a physical model as follows to elucidate the physical mechanisms. 

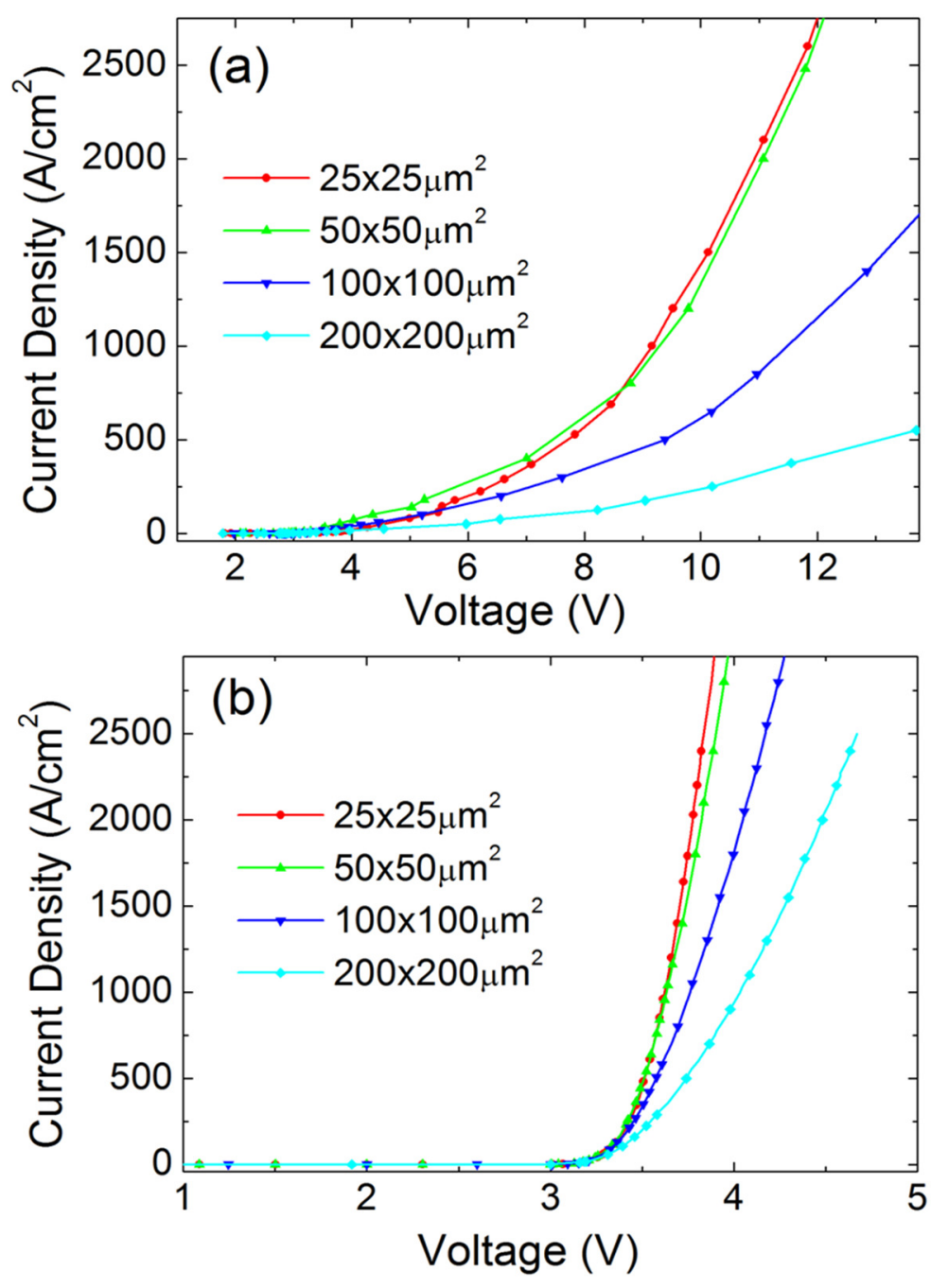

Figure 4.2 J-V diagrams of LTM-LEDs of different sizes for (a) experimental data; and (b) simulated data.

Figure 4.3(a) shows the schematic drawing of the current paths of the LTM-LED. The width and length of the mesa are both $L$. $L_{0}$ is the distance between the mesa edge and the n-contact edge. As the current is crowed at the edge of the n-contact, the resistance under the n-Pad has little impact on the LED performance. Hence, $\left(L_{k}\right.$ $\left.+L_{0}\right)$ is the $\mathrm{n}-\mathrm{GaN}$ distance across which $J_{k}$ is flowing before reaching the $\mathrm{n}$-contact. $H$ is the thickness of n-GaN after mesa etching. $T$ is the mesa depth which includes 
the p-GaN, the MQWs, and part of the n-GaN. In our devices, $L_{0}, H$, and $T$ are fixed for all these four LEDs with different sizes. To facilitate the analysis, we further simplified the equivalent circuit as shown in Figure 4.3(b), in which $R_{l}$ denotes the resistance along the vertical direction from the p-current spreading layer to the $n$ GaN and $R_{2}$ denotes the resistance corresponding to $L_{0}$. The current horizontally flowing along the $\mathrm{n}$-GaN can be divided into $\mathrm{m}$ sub-paths, and $r_{k}$ is the resistance corresponding to $L_{k}$ where $k=1,2, \ldots, m$-1, and $m$, in which $m$ is infinity. As we know, for a fixed bias voltage, the higher the resistance, the lower the current. Based on the above analysis, the current density is heavily influenced by $L$. Therefore we use the above model to deduce the relationship between the current density $J$ and $L$.

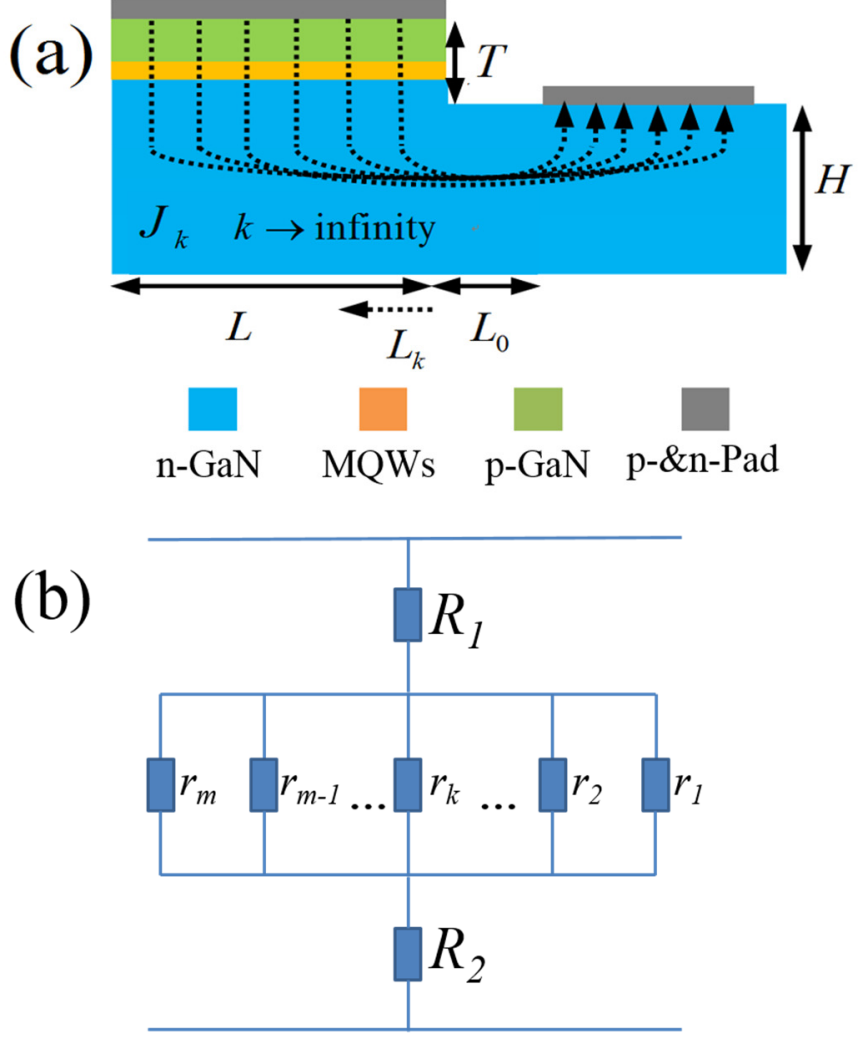

Figure 4.3 (a) Schematic current paths of the LTM-LED and (b) the simplified circuit model for the LTM-LED. 
First, we deduce the resistance $R_{1}, r_{k}$, and $R_{2}$ between the p-Pad and the n-pad.

$$
\begin{gathered}
R_{1}=\sum \rho_{i} \frac{T_{i}}{S_{1}} \\
r_{k}=\rho_{N-G a N} \frac{L_{k}}{S_{2}} \\
R_{2}=\rho_{N-G a N} \frac{L_{0}}{S_{2}}
\end{gathered}
$$

where $S_{l}=L^{2}$ is the mesa area, $S_{2}=L \times H, T=\sum T_{i}$ and $i$ refers to individual epitaxial layers, including p-GaN, MQW, and part of n-GaN.

Based on Eq. (4.1), (4.2), and (4.3) we can obtain the current density component $J_{k}$

$$
J_{k}=\frac{V}{\sum \rho_{i} T_{i}+\rho_{N-G a N} \frac{L}{H} \times\left(L_{k}+L_{0}\right)}=\frac{V}{R_{0}+a L\left(L_{k}+L_{0}\right)}
$$

where $R_{0}=\sum \rho_{i} T_{i}$ and $a=\frac{\rho_{N-G a N}}{H}$. Based on Eq. (4.4), we arrive at the average current density $J_{\text {ave }}$ :

$$
J_{\text {ave }}=\frac{1}{L} \int_{0}^{L} J_{k} d L_{k}=\frac{V}{a L^{2}}\left(\ln \left(a L^{2}+a L_{0} L+R_{0}\right)-\ln \left(a L_{0} L+R_{0}\right)\right)
$$

Here, $J_{\text {ave }}$ is the current density in our experiments and simulations. Eq. (4.5) reveals the relationship between the current density $J_{\text {ave }}$ and the edge length of the mesa $L$. To plot the $J_{\text {ave }}$ as a function of $L$, the parameters $a$ and $R_{0}$ appearing in Eq. (4.5) have to be estimated. Based on our experimental result $\rho_{N-G a N}=4.745 \times 10^{-3} \Omega \cdot \mathrm{cm}$, and $\mathrm{H}=5.5 \mu \mathrm{m}$, and therefore $a=8.627 \Omega . R_{0}$ is related to the material properties and device geometry and can be obtained from a specific experimental result. Taking the 
device of $50 \times 50 \mu \mathrm{m}^{2}$ as an example, when $V=11.073 \mathrm{~V}, J=2,000 \mathrm{~A} / \mathrm{cm}^{2}$, from Eq. (4.5) we find $R_{0}=5.343 \times 10^{-3} \Omega \cdot \mathrm{cm}^{2}$. Using the parameters above, the relationship between the current density and the mesa length $(J-L)$ is presented in Figure 4.4(a). Although $R_{0}$ has an influence on the absolute value of $J$, it will not change the trend of this relationship. From Figure 4.4(a), it can be seen that $J$ decreases with increasing $L$. That is to say, the smaller the size, the higher the current density, which may be caused by the increased non-uniformity of the current spreading in the larger sized LEDs [34]. This well matches the previously reported results [34] and our observations in Figure 4.2. As it is well known, the n-pad and the mesa have to be separated to prevent the side-wall current leakage. The distance $L_{0}$ between the npad and the mesa is an important parameter to be optimized as it also affects the characteristics of the current density as shown in Figure 4.4(b). It can be seen from Figure 4.4(b) that with the increasing $L_{0}$, the current density $J$ will decrease. This is because the resistance from n-pad to p-pad is increasing with $L_{0}$. Therefore, $L_{0}$ has to be optimized to fulfill the requirement of both preventing current leakage and increasing the current density. 

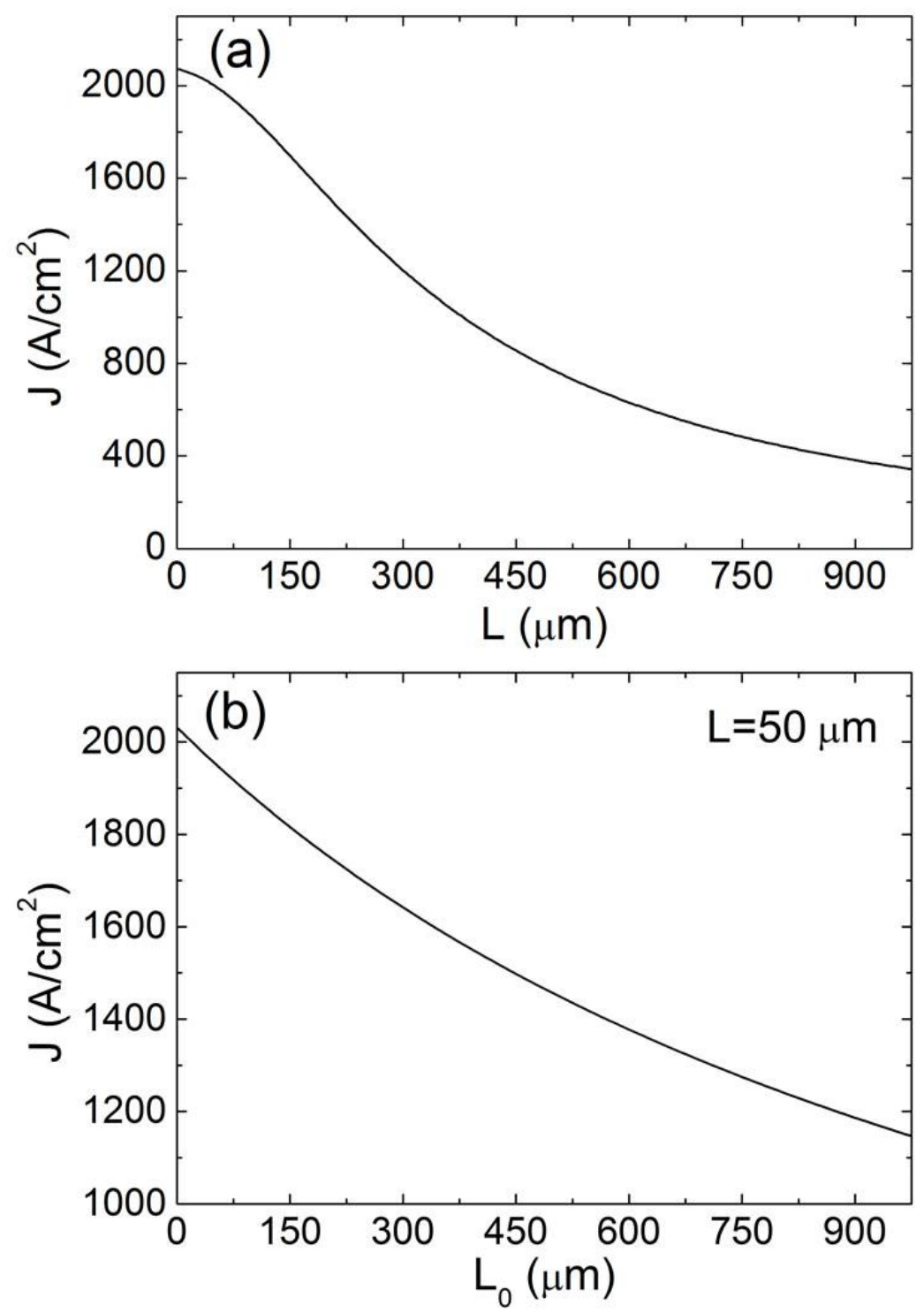

Figure 4.4 (a) $J-L$ and (b) $J-L_{0}$ behaviour at a fixed voltage bias $(11.073 \mathrm{~V})$.

It is worth noting that the $J$-V improvement in Figure 4.2 is less significant when the chip size is further scaled down from $50 \times 50 \mu \mathrm{m}^{2}$ to $25 \times 25 \mu \mathrm{m}^{2}$. This is different from the previously reported results [34], where the $J-V$ is improved consistently even when the chip size is smaller than $50 \times 50 \mu \mathrm{m}^{2}$. The reason for this difference is 
that the p-contact area was used as the LED effective area to calculate the current density in the previous reports while in our results the mesa area was used. Therefore, due to the fact that the p-contact area is always smaller than the mesa area (the edge of the mesa is not covered by the metal layer), there is a limit on the current density improvement with the decreasing device size and an optimal mesa size should exist and can be found out through numerical simulations. A further detailed discussion in this report will be conducted later.

Figures 4.5(a) and 4.5(b) show the experimentally measured and numerically simulated power density-current density $(P D-J)$ diagrams of the LTM-LEDs of different sizes, respectively. The simulated $P D-J$ characteristics of the studied chips exhibit the same trend as the experimental ones. It can be seen that the power density is increasing initially with the increasing current density and then starts saturating and decreasing after reaching a peak point as the current density further increases for the devices of $200 \times 200 \mu \mathrm{m}^{2}, 100 \times 100 \mu \mathrm{m}^{2}$ and $50 \times 50 \mu \mathrm{m}^{2}$. More importantly, the power density of the smaller chips is always larger than that of the larger chips in the whole current density range and the peak power density of the smaller chips is much larger than that of the larger chips at much larger current density. These characteristics of $P D-J$ of the different sized chips show that the smaller chips are much superior when operating at high current density and delivering high power density. The reason behind this is the low thermal mass of the smaller chips, storing less heat. As the LTM-LED size decreases, $r_{k}$ will become smaller. Accordingly, the heat generated by the additional $r_{k}$ is $I^{2} r_{k} t$. This decreased resistance will generate less Joule heat, which is the main reason for low thermal effect of the smaller chips. 
The external quantum efficiency (EQE) of the LEDs is adversely affected by the heat generated in the devices [119]. Since much less heat is generated in the LTM-LED chips, the EQE is preserved well even at high current density, and therefore a much improved power density is realized in the LTM-LEDs. It should be noted in Figures 4.5(a) and 4.4(b) that as the chip size further scales down from $50 \times 50 \mu \mathrm{m}^{2}$ to $25 \times 25$ $\mu \mathrm{m}^{2}$, the power density performance starts to degrade. This phenomenon can be explained by the difference of the areas of the current spreading layer and the mesa. As we have mentioned above that the side length of the current spreading layer is 10 $\mu \mathrm{m}$ shorter than that of the mesa, this means high resistance region exists in the margin area, which becomes critical in the very small device size. To find how the margin area affects the $25 \times 25 \mu \mathrm{m}^{2}$ device, we put forward a model to analyze the structure. 

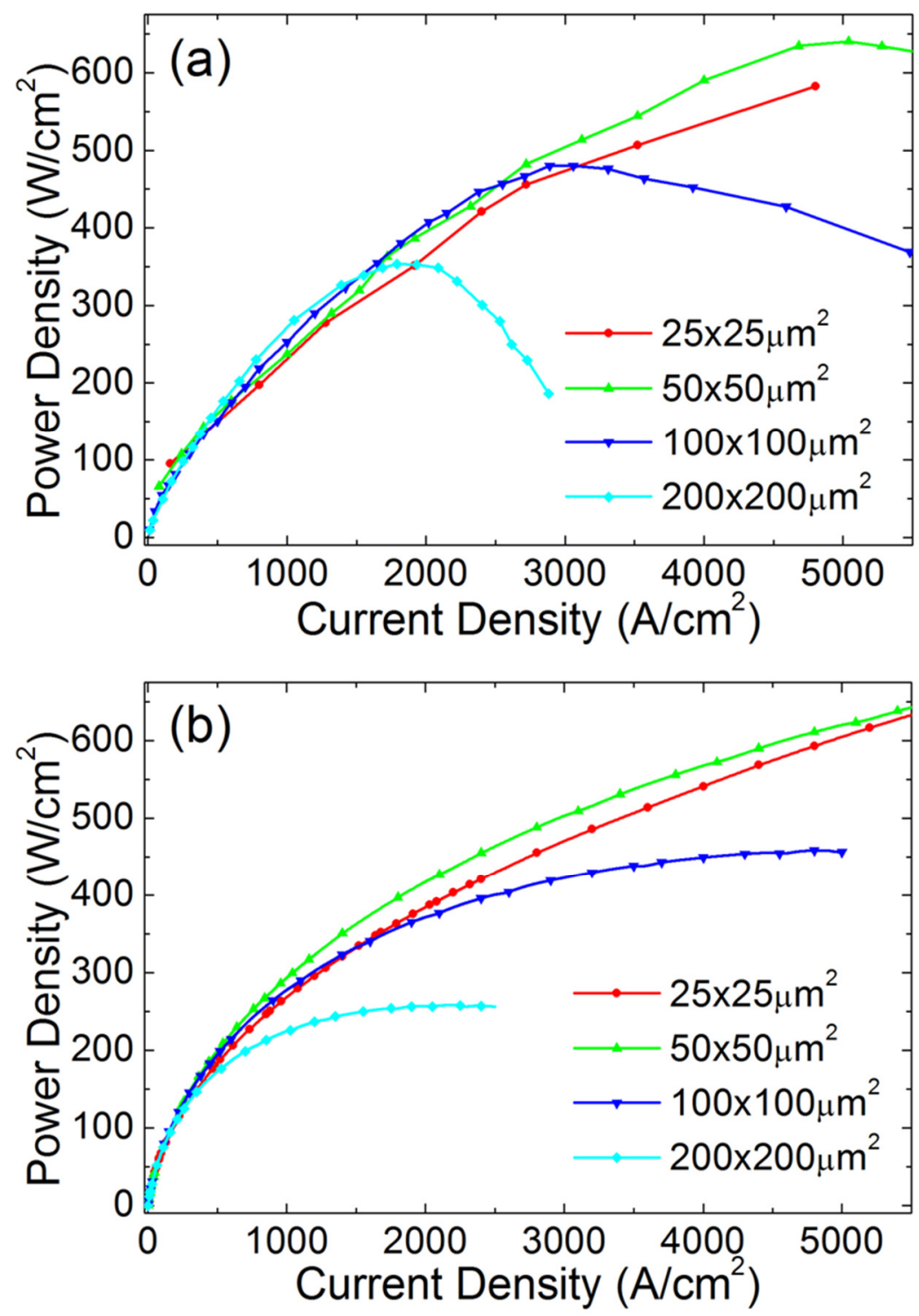

Figure 4.5 PD- $J$ diagrams of LTM-LEDs in different sizes: (a) experimental results and (b) simulation results.

Figure 4.6(a) shows the general view of the LTM-LED, especially the perceptual intuition of the current spreading layer and the mesa. Figure 4.6(b) shows the schematic diagram of the circuit of the different current path ways between the current spreading layer and the mesa. $R_{l}$ is the resistance of the p-pad, which is made 
of metal, so the resistance is very small. $R_{2}$ is the resistance of $\mathrm{p}-\mathrm{GaN}$ in the margin area. $R_{3}$ and $R_{4}$ are the resistance of $\mathrm{PN}$ junction, which is from $\mathrm{p}-\mathrm{GaN}$, through MQWs to n-GaN. $R_{5}$ and $R_{6}$ are the $\mathrm{n}-\mathrm{GaN}$ resistance from point $C$ to point $B$ and point $B$ to n-pad, for which the resistivity is much smaller than that of $\mathrm{p}-\mathrm{GaN}$. To compare the effect of the margin area, we select two current path ways from point $A$ to point $B$. One includes the resistance of $\mathrm{p}-\mathrm{GaN}$ in the margin area, and the other one is randomly selected just under the p-contact area: (1) is the path through $R_{1}, R_{2}$, and $R_{3}$, including p-pad, the margin area and $\mathrm{PN}$ junction, which has the current value of $I_{1}$; (2) is the path through $R_{4}$ and $R_{5}$, including PN junction and part of nGaN, which has the current value of $I_{2}$. If we consider the current ratio of (1) and (2)

$$
\frac{I_{1}}{I_{2}}=\frac{R_{4}+R_{5}}{R_{1}+R_{2}+R_{3}}
$$

From Eq. (4.6), we can see that $R_{2}$ is much larger than $R_{1}, R_{3}, R_{4}$, and $R_{5}$. Therefore, $I_{1}$ will be much smaller than $I_{2}$. This is the main reason for the similar current density versus bias voltage behavior for the devices with the size of $25 \times 25 \mu \mathrm{m}^{2}$ and $50 \times 50$ $\mu \mathrm{m}^{2}$. Due to the fabrication limitation that a margin area without the cover of current spreading layer is inevitable for a reliable device, with the decrease in the chip size, the current density improvement as a function of the bias voltage will be diminished. On the other hand, the ratio of the current spreading layer area to the mesa area for the $25 \times 25 \mu \mathrm{m}^{2}$ device is 0.36 , much smaller than that of $50 \times 50 \mu \mathrm{m}^{2}$ device which is 0.64. The mesa area without the current spreading layer cover has much lower optical output power density due to the lower current density. Therefore, the ratio of the 
effective radiation area is smaller for the $25 \times 25 \mu \mathrm{m}^{2}$ device. This leads to the degradation of the power density of the $25 \times 25 \mu \mathrm{m}^{2}$ device compared to that of the $50 \times 50 \mu \mathrm{m}^{2}$ size one.

(a)

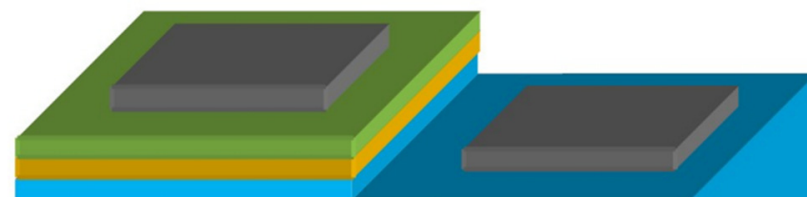

(a)
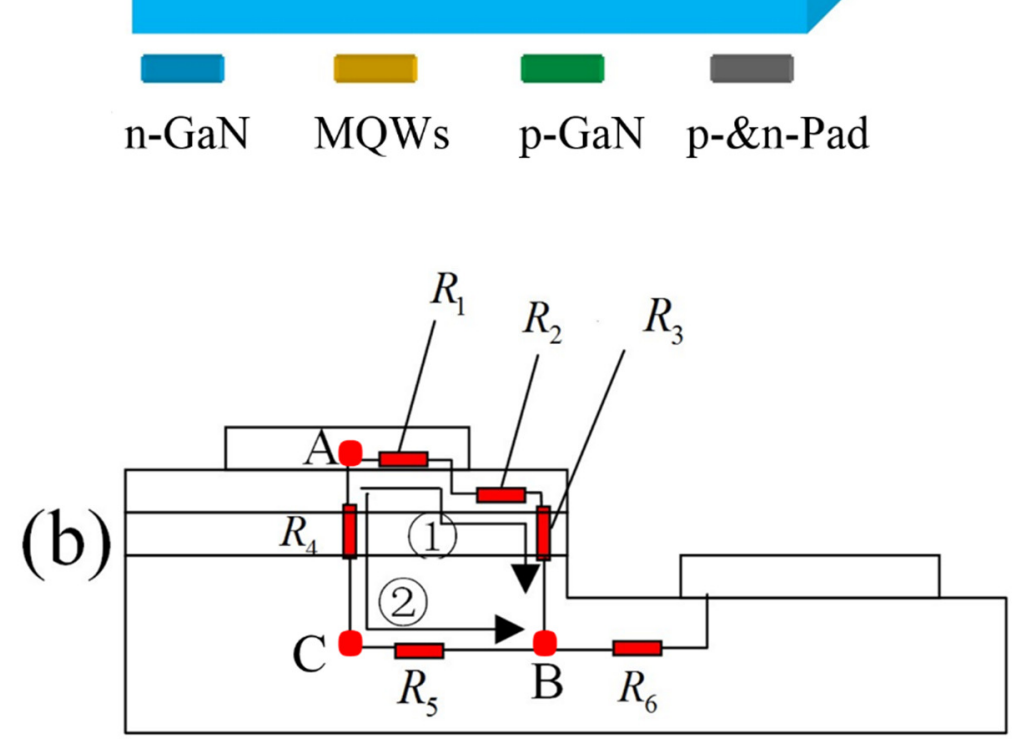

Figure 4.6 Schematic view of (a) LTM-LED current spreading layer and mesa margin and (b) the equivalent circuit model for the margin effect. 


\subsection{Conclusions}

In conclusion, the size effect and the fabrication limitation of LTM-LEDs have been experimentally and theoretically studied and understood. Both experimental and numerical results show that there exists an optimal mesa size for the current density and power density performance of the LTM-LEDs with the mesa area different from the current spreading layer area. Based on this work, as a design rule of thumb for high current density and high power density LTM-LEDs, three key points can be listed: first, the current spreading layer size should be made as close as possible to the mesa size; second, an optimum mesa size should be determined according to the specific layer structures and the design layout; third, the n-pad should be made as close to the p-pad as possible before any severe current leakage happens. 


\section{Chapter 5. Low thermal-mass LEDs: shape effects}

To fulfill the requirements of various applications, LEDs in different sizes and geometries have been designed. In the last chapter, we have systematically studied the size effect on the electrical and optical properties of LTM-LEDs and the optimized size for LTM-LEDs was found based on our fabrication conditions. In this chapter, we put our effort on the shape effect of LTM-LEDs. Here, triangular, circular, and square LTM-LEDs with the same area were designed and demonstrated in a flip-chip configuration. The shape effects on electrical and optical characteristics of these LTM-LEDs were systematically studied both theoretically and experimentally. Here it was revealed that the triangular LTM-LEDs deliver the highest current as a function of the bias voltage and the highest output power as a function of the current, whereas the circular ones exhibit the lowest of both, among the three types of LTM-LEDs. Regarding the shape effect, although all the previous reports use broad-area LEDs (and not a LTM-LED) and mainly focus on the enhancement in light extraction from the sidewalls, here our numerical simulations demonstrated that the light extraction efficiency of the triangular LTM-LEDs is only $0.06 \%$ higher than the circular ones. Experimentally, however, the triangular devices yield a $33.4 \%$ higher output power than the circular ones and $18.9 \%$ better than the square ones at $200 \mathrm{~mA}$. Here, based on our model and numerical simulations, we uncovered the underlying physical mechanisms of these observations and found that the reduced current crowding and self-heating at the mesa edge, and shorter n-GaN current paths contribute to the superior performance of the triangular LTM-LEDs. These findings indicate that such carefully shaped LTM-LEDs hold great promise for high-speed modulation in VLC and micro-displays.

\subsection{Introduction}

Since InGaN/GaN-based LEDs were invented, both IQE and light extraction efficiency (LEE) of LEDs have been improved dramatically [55, 56, 112, 120]. Up to date, the applications of high-power LEDs are being deployed into many different 
platforms, such as medical lighting [121], micro-displays [24], visible light communication [122], and biosystems [123]. Especially in the last decade, smart LEDs [17], which offer controllability of their spatial, temporal, spectral, color temperature, and polarization properties, attract much attention in both research fields and our daily life. To make LEDs smart, LEDs with high current density, high power density, and high modulation response speed are required [107]. For example, visible light communication, which may provide high data rate indoor communication and high accuracy object positioning in retail stores, need LEDs with current density above $500 \mathrm{~A} / \mathrm{cm}^{2}$ to increase the modulation frequency and with high power density to attain the primary illumination function. In this regard, broad area LEDs with square size, which typically deliver current density in the range of 1 $\mathrm{A} / \mathrm{cm}^{2}$ to $100 \mathrm{~A} / \mathrm{cm}^{2}$, are not effective for these applications. Thus, LTM-LEDs, which can deliver much higher current density and power density than general broad area LEDs, were proposed, and the size effects of LTM-LEDs have been studied and reported [107]. It is suggested that the less thermal mass and less Joule heat generated by the LTM-LEDs are the underlying benefits of LTM-LEDs [107]. However, the shape effects of LTM-LEDs are still unknown.

In terms of broad area LEDs, there have been several studies on the shape effects [124-126]. Kim et al. demonstrated that the total power of a triangular LED increased by $24 \%$ at $100 \mathrm{~mA}$ compared with a standard quadrangular one [124]. Wang et al. reported that the circular geometry shows an enhancement of overall light extraction due to the rotational symmetry of the geometry [125]. Wang et al. also studied the contribution of sidewall light extraction to the total power of polygonal LEDs and 
the results show that triangle- and pentagon-shaped LEDs have the highest power [126]. In these studies, the authors focused mainly on the light extraction [124-126]. However, the $\mathrm{n}-\mathrm{GaN}$ resistance from the n-contact to the LED mesa has an essential influence on the current spreading and output power of the LEDs [30]. This places a constraint, limiting the potential improvement achievable with the shape effects. Nevertheless, previous works did not study these issues.

In this work, we grew and fabricated novel structured LTM-LEDs for which the mesa is surrounded by the n-pad to achieve high current density and power density. Furthermore, these LTM-LEDs were designed in three different shapes with the same mesa area: (1) triangular LTM-LEDs with a mesa side length of $303.9 \mu \mathrm{m}$, (2) circular LTM-LEDs with a mesa diameter of $225.7 \mu \mathrm{m}$, and (3) square LTMLEDs with a mesa side length of $200 \mu \mathrm{m}$. In our three LTM-LEDs, the depth of the side-wall is $800 \mathrm{~nm}$, which is much smaller than that $80 \mu \mathrm{m}$ (the sapphire thickness after dicing) reported by Kim et al. [124]. We experimentally found $33.4 \%$ improvement at the output power from the triangular ones at $200 \mathrm{~mA}$ compared to the circular ones. We systematically investigated the LEE of our structures with Monte-Carlo method. The LEE of the triangular LTM-LEDs is only $0.06 \%$ higher than that of the circular LTM-LEDs as the sidewall depth is too shallow. However, this $0.06 \%$ improvement cannot explain the $33.4 \%$ improvement of the output power in our experimental results. This phenomenon and its physical reasoning have not been reported to date to the best of our knowledge and needs a systematical investigation and demonstration. Here, we systematically studied the n-GaN resistance effect on current and output power. Our model and the numerical APSYS 
simulations demonstrate that because of the lower resistance at the mesa edge and the shorter $\mathrm{n}-\mathrm{GaN}$ current paths, the Joule heating is reduced and the average radiative recombination rate is enhanced, and hence the triangular LTM-LEDs have the highest current as a function of the voltage and the largest output power as a function of the current.

\subsection{Growth, fabrication and simulations of different shaped}

\section{LTM-LEDs}

In order to be consistent with the conditions set in our numerical simulations with Monte-Carlo method, C-plane double polished sapphire substrates were used to grow the LED epitaxial wafers by the MOCVD system. There are 6 quantum wells in the wafer, and the other details of the growth process can be found in "3.2. Epitaxial structure of InGaN/GaN-based LEDs" in Chapter 3.

Following the MOCVD growth, standard micro fabrication techniques are used to fabricate the LED wafers into the flip-chip configuration. Mesa shapes of triangle (side length $303.9 \mu \mathrm{m}$ ), circle (diameter $225.7 \mu \mathrm{m}$ ), and square (side length $200 \mu \mathrm{m}$ ) were prepared using reactive ion etching (RIE) on the same wafer. Ni/Ag-based metal layers, which were annealed in oxygen ambient after being deposited, were used as the reflective mirror. $\mathrm{Ti} / \mathrm{Au}(30 \mathrm{~nm} / 1,000 \mathrm{~nm}$ thick) metal layers were deposited by an e-beam evaporation as the p- and n-contact pads. The mesa shapes were surrounded by n-contact pads $15 \mu \mathrm{m}$ off the mesa edge. A LED tester (M2442S9A Quatek Group) was used to measure the current-voltage characteristics of the 
LED chips and the optical output power was measured by an Ocean Optics spectrometer (QE65000) attached to an integrating sphere.

Monte-Carlo method was used to study the LEE of different shaped LTM-LEDs. In the simulations, all surfaces of the LEDs are modeled to be of optical quality. The Snell's law applies when light travels through the materials and across interfaces. The angular of the light emitted from the MQWs is uniformly distributed. The optical properties at $450 \mathrm{~nm}$ adopted in the simulations are as follows: double polished sapphire substrate (thickness $420 \mu \mathrm{m}$, refractive index 1.77), U-GaN (thickness 3 $\mu \mathrm{m}$, refractive index 2.4), $\mathrm{n}-\mathrm{GaN}$ (thickness $5.5 \mu \mathrm{m}$, refractive index 2.43), MQW (thickness $0.09 \mu \mathrm{m}$, refractive index 2.54, absorption 2,000/cm), AlGaN (thickness $0.02 \mu \mathrm{m}$, refractive index 2.42) and $\mathrm{p}-\mathrm{GaN}$ (thickness $0.2 \mu \mathrm{m}$, refractive index 2.45, absorption 2,000/cm) [127]. The optical flux emitted from the MQW was set at $1 \mathrm{~W} / 1,000,000$ rays for this simulation [126].

To understand the effect of the distance from n-electrode to LED mesa on the electrical and optical properties, a numerical modeling was performed. In our simulations, we used the APSYS software to self-consistently solve the Poisson equation, the continuity equation, and Schrödinger equation with proper boundary conditions. More details on the simulation can be found in "4.2. Growth, fabrication and simulation of different sized LTM-LTDs" in Chapter 4. 


\subsection{Experimental and simulated results and discussion}

Table 5.1 shows the LEE for the triangle-, circle-, and square-shaped LTMLEDs based on Monte-Carlo method. From Table 5.1, it is clear that the LEE of the triangular LTM-LEDs is slightly higher than the circular and square LTM-LEDs. Through calculation, the LEE of the triangular LTM-LEDs is only $0.06 \%$ higher than the circular LTM-LEDs. These results indicate that the LEE is nearly the same for LTM-LEDs with different shapes, since the side-wall of our LTM-LEDs is only 800 $\mathrm{nm}$, which can be negligible compared with the $80 \mu \mathrm{m}$ side-wall reported in previous work [124].

Table 5.1 LEE of triangle-, circle-, and square-shaped LTM-LEDs based on Monte-Carlo methods.

\begin{tabular}{|c|c|c|c|}
\hline Shape & Triangle & Circle & Square \\
\hline LEE & $16.84 \%$ & $16.83 \%$ & $16.85 \%$ \\
\hline
\end{tabular}

On the other hand, the optical and electrical performance of these LTM-LEDs shows great dependence on shapes. Figure 5.1(a) shows the current-voltage $(I-V)$ characteristics of different shaped LTM-LEDs with the same mesa area. It can be seen that the triangular LTM-LEDs have the highest current against voltage while the circular LTM-LEDs show the lowest. Figure 5.1(b) shows the experimental measurement for power-current $(P-I)$ for triangular, circular and square LTM-LEDs with the same area. Similarly, Figure 5.1(b) depicts that the optical power of the triangular LTM-LEDs is the highest whereas that of the circular LTM-LEDs is the lowest at a given current. After calculation, we found that the triangular LTM-LEDs 
exhibit a $33.4 \%$ higher optical power than the circular ones and $18.9 \%$ higher than the square ones at $200 \mathrm{~mA}$. Apparently, this huge improvement cannot be attributed to the LEE, as discussed previously, and the physical reason of this phenomenon has never been reported.
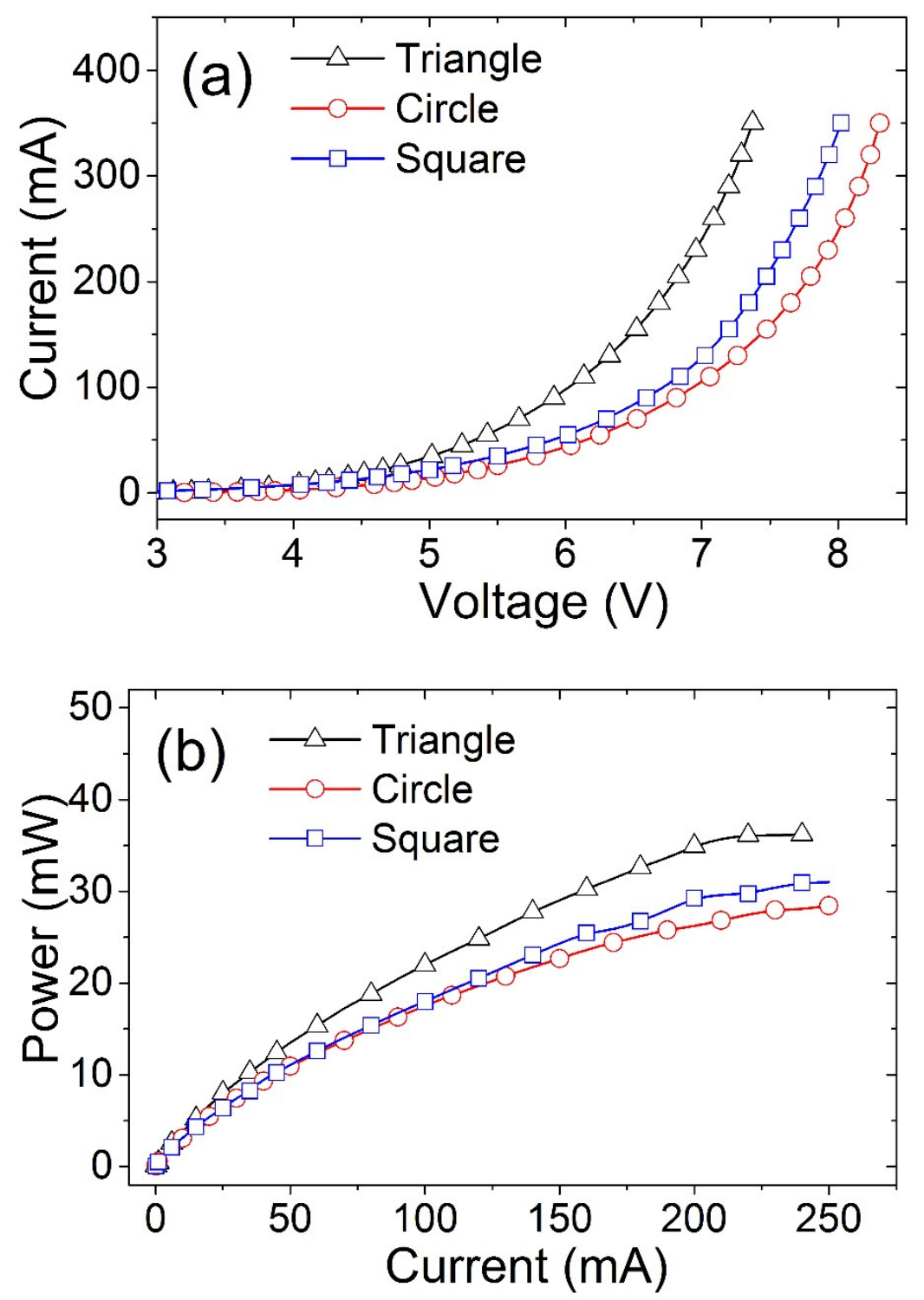

Figure 5.1 Experimental results of (a) $I-V$ and (b) $P-I$ diagrams of the triangular, circular, and square LTM-LEDs with the same mesa area. 
To understand the observed phenomenon in the $P-I$ and $I-V$ characteristics from the internal device perspective, the current paths of the LTM-LEDs are depicted in Figure 5.2. From Figure 5.2, we can see that the current from p-pad finally all flows to n-pad through the dashed area A, where will be a bottleneck of the current flow. Thus, the resistance $R$ in area $\mathrm{A}$ is critical for the bias voltage and the Joule heating of the LTM-LEDs. $R=\rho L_{l} / S$, in which $\rho$ is the $\mathrm{n}-\mathrm{GaN}$ resistivity, $L_{l}$ is the current paths from the mesa edge to n-pad, $S$ is the cross section area of the current flow along $L_{1}$. For the three shaped LTM-LEDs, in Figure 5.2 area A, $\rho$ and $L_{l}$ are the same. As the p-pad is surrounded by the n-pad as shown in Figure 5.3, $S=h \times p$, in which $h$ is the $\mathrm{n}-\mathrm{GaN}$ thickness under the $\mathrm{n}-\mathrm{pad}$ as shown in Figure 5.2, and $p$ is the perimeter for different shapes. For the triangular LTM-LEDs, $p=3 \times 303.9 \mu \mathrm{m}=911.7$ $\mu \mathrm{m}$; for the square LTM-LEDs, $p=4 \times 200 \mu \mathrm{m}=800 \mu \mathrm{m}$; for the circular LTM-LEDs, $p=\pi \times 225.7 \mu \mathrm{m}=708.7 \mu \mathrm{m}$. Thus, in area $\mathrm{A}$, the resistances of triangular, square, and circular LTM-LEDs are $R_{t}=\rho L_{1} /(h \times 911.7 \mu \mathrm{m}), R_{s}=\rho L_{l} /(h \times 800 \mu \mathrm{m}), R_{c}=$ $\rho L_{1} /(h \times 708.7 \mu \mathrm{m})$.

For the bias voltage, the voltage drop in area $\mathrm{A}$ is an important part. As $V=I R$, and $R_{t}$ is the smallest and $R_{c}$ is the largest, the triangular LTM-LEDs have the smallest voltage drop and the circular ones have the largest voltage drop in area A, which matches well with the voltage performance of these LTM-LEDs as shown in Fig. 5.1(a). 


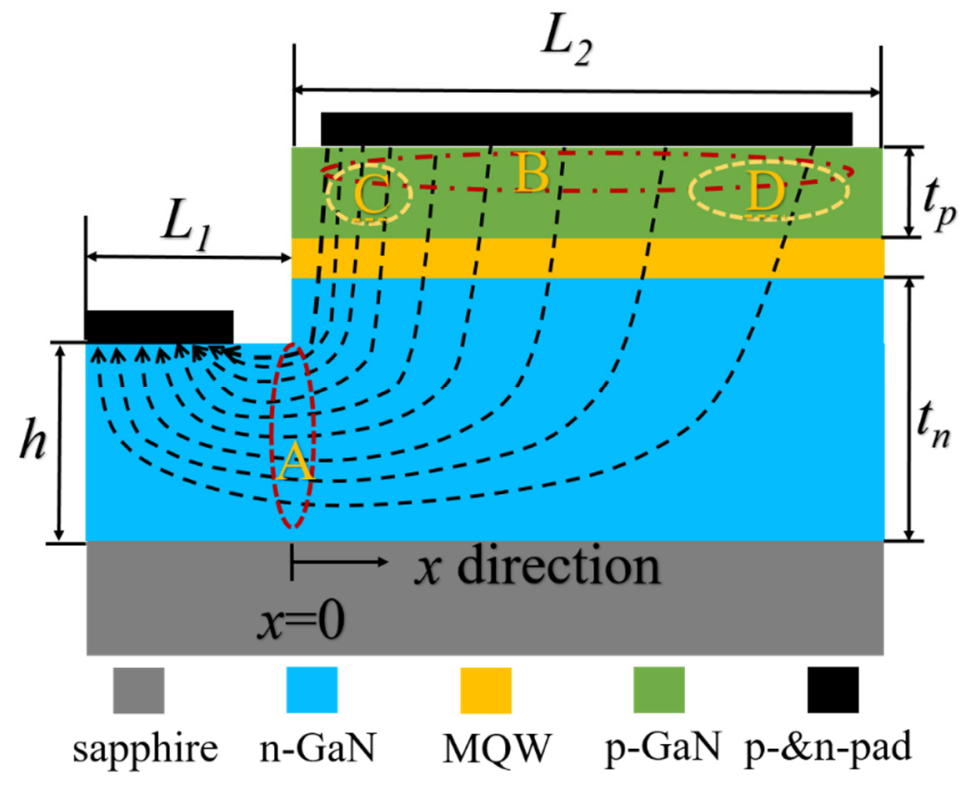

Figure 5.2 Illustration of current paths of the LTM-LEDs with a flip chip structure.

For the optical performance, there are several mechanisms. Firstly, based on the Joule heating $J=I^{2} R t$, under the same current $I$, the ratio of the heat generated by the circular, square, and triangular LTM-LEDs in area $\mathrm{A}$ is $I^{2} R_{c} t: I^{2} R_{s} t: I^{2} R_{t} t=1.29$ : $1.14: 1$. After calculation, we found that the heat generated in area A by circular LTM-LEDs is $29 \%$ higher than the triangular ones, and that of the square ones is $14 \%$ higher than the triangular ones. As the external quantum efficiency (EQE) of the LEDs is adversely affected by the heat generated in the devices [128] and much less heat is generated in the triangle-shaped LTM-LED chips, the EQE is preserved well even at high current, and therefore a much improved power is realized in the triangle-shaped LTM-LEDs. On the other hand, this lowest resistance due to the longest mesa edge reduces the current crowding effect at the mesa edge for triangular 
LTM-LEDs compared to the square and circular ones, which also leads to a high output power.

To compare the current path $L_{2}$ as shown in Figure 5.2, the current paths of the LTM-LEDs are depicted on the microscopic pictures of the LTM-LEDs in Figure 5.3. As the mesa shapes are surrounded by n-electrodes $15 \mu \mathrm{m}$ off, from the mesa edge, the shortest current paths are directed vertically toward the n-electrode, as shown in Figures 5.3(a), 5.3(b) and 5.3(c). For Figures 5.3(a) and 5.3(b), we divided the triangular LTM-LED into six unit cells and the square one into eight unit cells, respectively. From the current paths of each unit cell, we can clearly see that the current paths of the triangular LTM-LEDs unit cell are shorter in general than those of the square and circular one, which will lead to a high output power. To further find the underlying reasons and demonstrate these mechanisms, we performed a series of APSYS simulations based on Figure 5.2. In the APSYS simulation, we studied effect of the $L_{2}$ in Figure 5.2, which is the longest current path from the center to the mesa edge for different sizes.
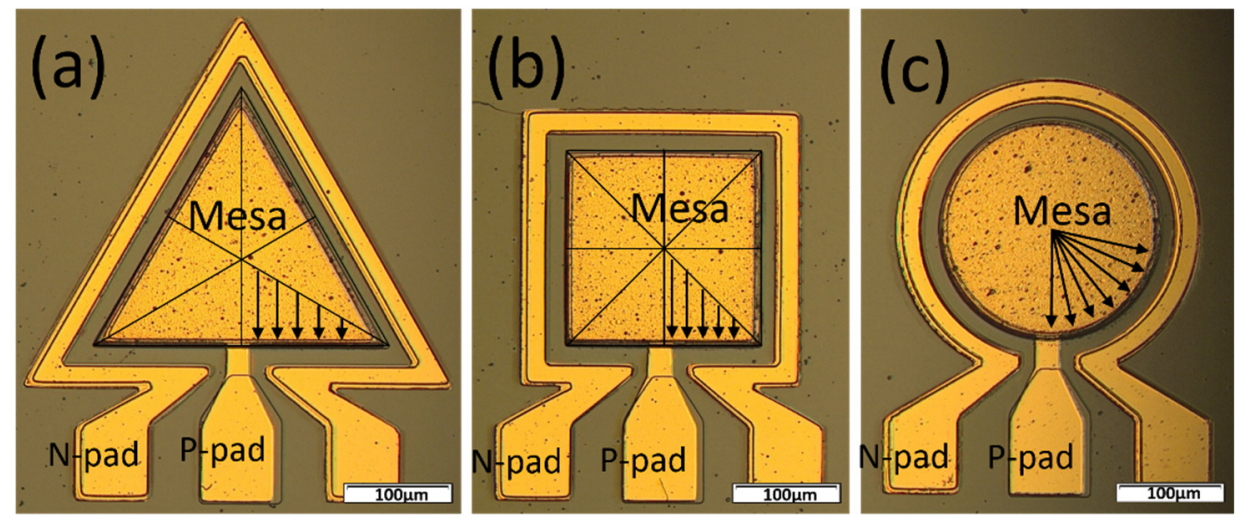

Figure 5.3 The microscopic pictures of (a) triangle-, (b) square-, and (c) circle-shaped LTM-LEDs with the same mesa area, on which the black 
arrows are schematic current paths.

Figure 5.4(a) presents the simulated results of $I-V$ characteristics for the LTMLEDs, in which the current of the triangular LTM-LEDs $\left(L_{2}=87.74 \mu \mathrm{m}\right)$ is the highest and that of the circular LTM-LEDs $\left(L_{2}=112.84 \mu \mathrm{m}\right)$ is the lowest at a given voltage. Figure 5.4(b) illustrates the simulated results of $I-P$ characteristics for the LTM-LEDs, in which the triangular LTM-LEDs $\left(L_{2}=87.74 \mu \mathrm{m}\right)$ show the highest output power and the circular ones $\left(L_{2}=112.84 \mu \mathrm{m}\right)$ deliver the lowest output power. Both of the simulated results are consistent with the experimental results. 

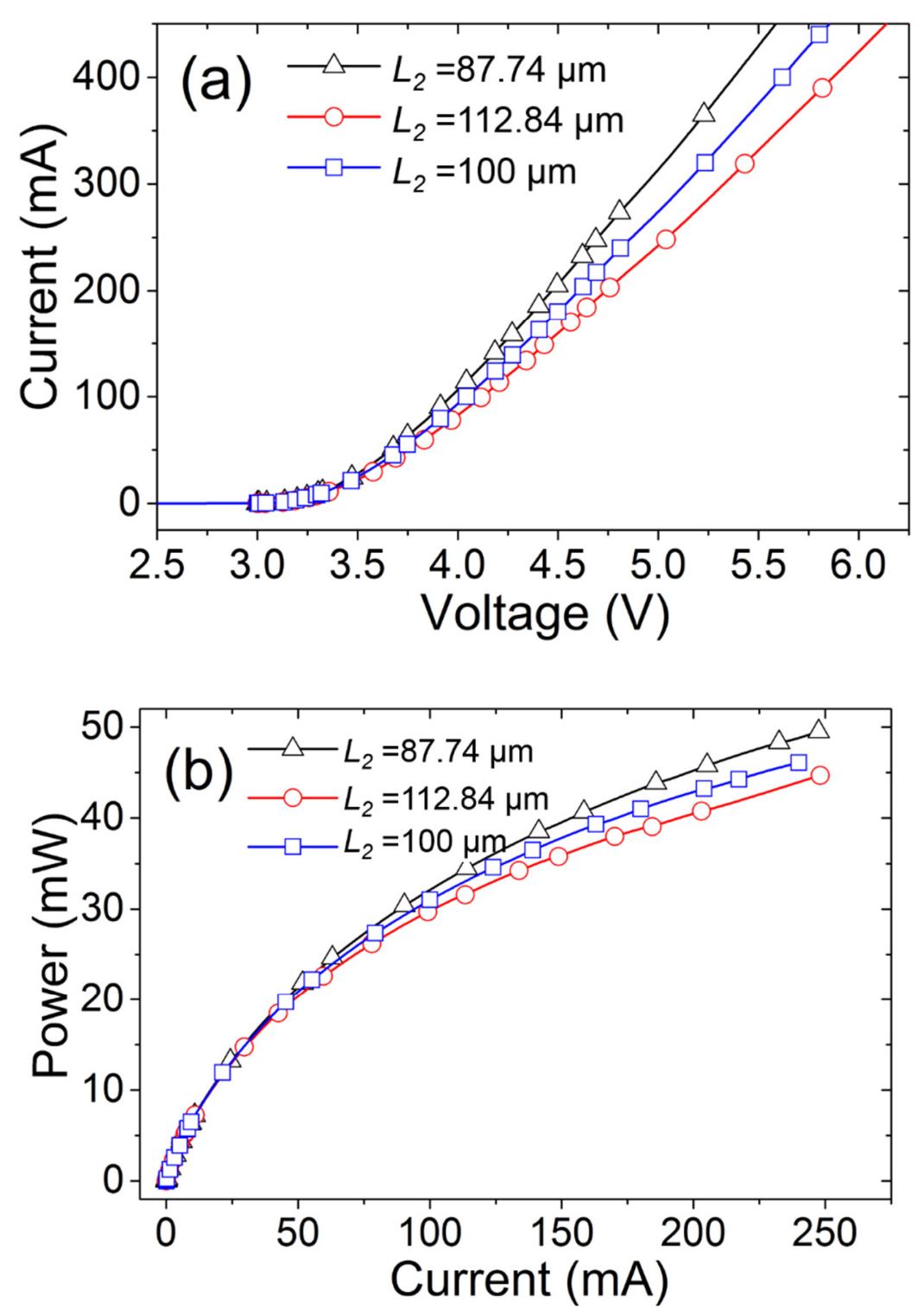

Figure 5.4 (a) $I-V$ and (b) $I-P$ characteristics of the triangular $\left(L_{2}=\right.$ $87.74 \mu \mathrm{m}), \operatorname{circular}\left(L_{2}=112.84 \mu \mathrm{m}\right)$, and square $\left(L_{2}=100 \mu \mathrm{m}\right) \mathrm{LTM}$ LEDs with the same area based on numerical simulation. $L_{2}$ is the longest current path from the center to the mesa edge.

Figures 5.5(a), 5.5(b) and 5.5(c) show the simulated results of electron \& hole concentrations and radiative recombination rates profile in the MQWs active region 
for the LTM-LEDs at different distances $(x=5 \mu \mathrm{m}, x=15 \mu \mathrm{m}, x=29.5 \mu \mathrm{m}, x=53.75$ $\mu \mathrm{m}, x=102.5 \mu \mathrm{m}, x=200 \mu \mathrm{m})$ at $200 \mathrm{~mA}$. The electron and hole concentrations for the LTM-LEDs, as depicted in Figures 5.5(a) and 5.5(b), decrease as $x$ increases, which leads to a declining recombination rate, as shown in Figure 5.5(c). To facilitate the comparison, we present the radiative recombination rates for different shapes of the LTM-LEDs in the last quantum well (near $\mathrm{p}-\mathrm{GaN}$ ) as a function of distance $x$ in one plot, as shown in Figure 5.5(d), also giving the calculated average values. From Figure 5.5(d), it can be observed that when the distance is shorter than $15 \mu \mathrm{m}$, which corresponding to area $\mathrm{C}$ in Figure 5.2, the triangular LTM-LEDs show the highest radiative recombination rate, and those of the square ones and circular ones are in a deep recession. This phenomenon can be explained by the current crowding effect. As discussed previously, the high current density occurs near the mesa edge and the length of the mesa edge is determined by the perimeter of different shapes, the shorter the perimeter, the severer the current crowding near the mesa edge. Triangular LTM-LEDs has the longest mesa edge $(p=911.7 \mu \mathrm{m})$ compared to the square $(p=800 \mu \mathrm{m})$ and circular $(p=708.7 \mu \mathrm{m})$ LTM-LEDs, which reduces the current crowding effect and leads to a higher radiative recombination rate. On the other hand, from Figure 5.5(d), when the distance is longer than $45 \mu \mathrm{m}$, which corresponding to area D in Figure 5.2, the triangular LTM-LEDs have the highest radiative recombination rate. Compared to the triangular LTM-LEDs, the circular ones have the longest current path from the mesa edge to the center $\left(L_{2}\right.$ in Figure 5.2), so the current density in Figure 5.2 area $\mathrm{D}$ is too low and the radiative recombination rate is the lowest. Thus, the average radiative recombination rate of 
the triangular LTM-LEDs is the highest, as can be seen from Figure 5.5(d), in which $0.234 \times 10^{28} \mathrm{~cm}^{-3} \mathrm{~s}^{-1}, 0.18 \times 10^{28} \mathrm{~cm}^{-3} \mathrm{~s}^{-1}$, and $0.202 \times 10^{28} \mathrm{~cm}^{-3} \mathrm{~s}^{-1}$ are the average radiative recombination rates for the triangular, circular, and square LTM-LEDs, respectively. After calculation, we found that the average radiative recombination rate of the triangular LTM-LEDs is $30.0 \%$ higher than the circular ones and $15.8 \%$ higher than the square ones at $200 \mathrm{~mA}$. Hence, we can conclude that the enhanced average radiative recombination rate and reduced Joule heating are the main reasons for the improvement of output power for the triangular LTM-LEDs.
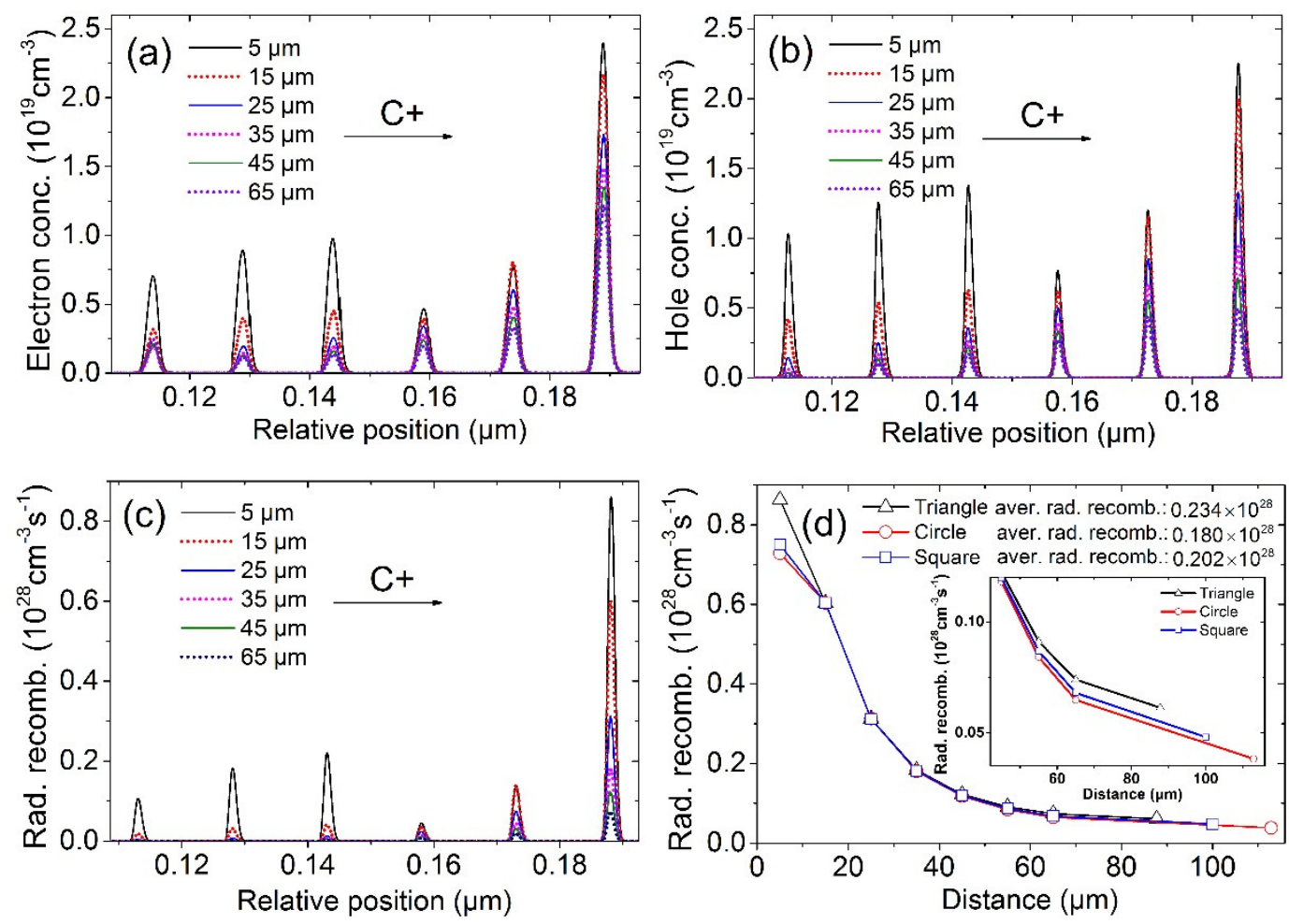

Figure 5.5 Simulated results for the LTM-LEDs at different distances ( $x=5 \mu \mathrm{m}, x=15 \mu \mathrm{m}, x=25 \mu \mathrm{m}, x=35 \mu \mathrm{m}, x=45 \mu \mathrm{m}, x=65 \mu \mathrm{m}$ ) at $200 \mathrm{~mA}$ based on Figure 5.2: (a) electron concentration; (b) hole concentration; and (c) radiative recombination rates. (d) The radiative recombination 
rates of the triangular, circular, and square LTM-LEDs in the last quantum well (near p-GaN) as a function of the distance to the mesa edge $(x=0)$, in which $0.234 \times 1028 \mathrm{~cm}^{-3} \mathrm{~s}^{-1}, 0.18 \times 1028 \mathrm{~cm}^{-3} \mathrm{~s}^{-1}$, and $0.202 \times 1028 \mathrm{~cm}^{-3} \mathrm{~s}^{-1}$ are the average radiative recombination rates for the triangular, circular, and square LTM-LEDs, respectively. In Figures 5.5 (a), (b), and (c), C+ indicates the growth orientation [0001] of the GaN.

\subsection{Conclusions}

In this article, triangular, circular, and square LTM-LEDs were designed and realized in the flip-chip configuration. The shape effects on electrical and optical characteristics of these LTM-LEDs were systematically studied both theoretically and experimentally. It was revealed that the triangular LTM-LEDs exhibit superior performance. It was found that the longer perimeter and shorter $\mathrm{n}-\mathrm{GaN}$ current paths account for the enhanced performance of the triangular architecture, which not only reduces the Joule heating but also contributes to the lower bias voltage. Moreover, with the increasing distance from the point on the LEDs mesa to the n-electrode, both the electron and hole concentrations decrease, which leads to a steep fall in the radiative recombination rates. As the perimeter is the longest and the perpendicular distance between the mesa center and the nearest mesa edge is the shortest, the triangular LTM-LED holds the highest average radiative recombination rate, which enables the triangular LTM-LEDs the highest output power. This high output power 
of the LTM-LEDs is essential for the variable applications of VLC and microdisplays, such as applications in large rooms, in the outdoor ambient and for the automotive headlight. On the other hand, this triangular shape of the LTM-LEDs, allowing for high current density, have a great potential to further help with increased modulation and control speed in the applications of VLC and micro-displays, which will be our future work. 


\section{Chapter 6. Size effects on the optical properties of low thermal-mass LEDs by partitioned growth}

To get the optimal output power of LTM-LEDs, the fabrication technique was used to make LTM-LEDs in different sizes and shapes. We found that the $50 \mu \mathrm{m}$ size and the triangle shape delivered the highest output power, respectively. To further increase the output power and at the same time grow the desired size of LTM-LEDs, we put forward the partitioned growth LTM-LEDs. In this chapter, low thermal-mass LEDs (LTM-LEDs) in $50 \times 50 \mu \mathrm{m}^{2}, 100 \times 100 \mu \mathrm{m}^{2}, 200 \times 200 \mu \mathrm{m}^{2}, 500 \times 500 \mu \mathrm{m}^{2}$ and $1,000 \times 1,000 \mu \mathrm{m}^{2}$ sizes were grown on patterned c-plane sapphire substrate with the metal-organic chemical-vapor deposition (MOCVD) technique, i.e., partitioned growth. The size effect on the optical properties and the indium concentration for the quantum wells were studied experimentally. It is revealed that the optical properties can be improved by decreasing the chip size (from $1,000 \mu \mathrm{m}$ to $100 \mu \mathrm{m}$ ), which can correspondingly reduce the in-plane compressive stress. However, when the chip size is further reduced to $50 \times 50 \mu \mathrm{m}^{2}$, the benefit of strain release is compensated by more defects induced by the higher indium incorporation in the quantum wells and the efficiency of the device decreases. The underlying mechanisms of the output power changing are uncovered based on different methods of characterization. In addition, this work also shows the rules of thumb to achieve optimal power performance for LTM-LEDs through a specific partitioned growth process. 


\subsection{Introduction}

To improve the external quantum efficiency (EQE) and optical output power, the GaN-based LEDs were extensively studied [55, 62, 112, 129]. Owing to the high efficiency, long lifetime, and versatile packages, etc, InGaN/GaN-based LEDs have been regarded as one of the most promising artificial lighting sources to substitute the incandescent and fluorescent lighting bulbs today. However, the performance of current commercial InGaN/GaN-based LEDs grown on the c-plane sapphire substrates is limited by the quantum confined Stark effect (QCSE) [130]. It is suggested that the strain induced large piezoelectric and spontaneous polarization fields contribute to the QCSE [131]. To overcome this issue, numerous efforts have been made to release the strain. For example, nonpolar tetragonal $\mathrm{LiAlO}_{2}$ substrate is used to reduce the QCSE by Waltereit et al. [132]. InGaN/GaN multiquantum well (MQW) nanorod arrays are implemented by Kim et al. to improve the efficiency of LEDs [133]. Substrates such as semi-polar and non-polar bulk GaN have ever been used by Nakamura et al. to achieve high IQE and low droop LEDs [134, 135]. However, these proposed methods come at high-cost. Therefore, in this work, we propose a low-cost and effective way to release the strain for achieving highefficiency LEDs through partitioned growth.

III-nitride based LEDs have been widely used as the backlighting for computers, flat-panel displays, and smartphones, and all these applications require small LED chips. As a result, small LEDs that can withstand high current density and deliver high power density are imperatively needed. To address these issues, low thermalmass LEDs (LTM-LEDs) have been proposed and reported by us [107]. LTM-LEDs 
can deliver much higher current density and power density than general broad area LEDs. The underneath benefits of LTM-LEDs are the less thermal mass and less Joule heat generated by the LTM-LEDs. Nevertheless, there is still much room to further improve the performance of LTM-LEDs as the growth process has not been optimized and the efficiency is still limited by the QCSE. On the other hand, it is deemed that if the LTM-LEDs can be directly in situ grown in the MOCVD chamber by adopting the partitioned growth process, i.e., partitioned growth LTM-LEDs (PG LTM-LEDs), which is unique and novel, the QCSE can be reduced.

In this work, LTM-LEDs with the sizes of $50 \times 50 \mu \mathrm{m}^{2}, 100 \times 100 \mu \mathrm{m}^{2}, 200 \times$ $200 \mu \mathrm{m}^{2}, 500 \times 500 \mu \mathrm{m}^{2}$, and $1,000 \times 1,000 \mu \mathrm{m}^{2}$ were grown on patterned c-plane sapphire substrate by using the MOCVD system (details on the growth process will be discussed subsequently). To protect these partitioned growth LTM-LEDs (PG LTM-LEDs) during the dicing process, a $10 \mu \mathrm{m}$ margin is needed between two individual chips. We investigated the size effect on the strain, the electrical and optical properties for the PG LTM-LEDs, which are critical to optimize the size for PG LTM-LEDs.

\subsection{Experiments on growth and characterization}

The LED epitaxial wafers were grown on c-plane single polished sapphire substrates by MOCVD. The sapphire substrate was firstly covered by $\mathrm{SiO}_{2}(100 \mathrm{~nm})$ by plasma enhanced chemical vapour deposition (PECVD), and the $\mathrm{SiO}_{2}$ layer was then patterned and etched with reactive-ion etching (RIE) to get square opening 
regions with different sizes. The margin width between two opening regions is 10 $\mu \mathrm{m}$. The wafer used in this chapter has 8 quantum wells, and more details on the growth process can be found in "3.2. Epitaxial structure of InGaN/GaN-based LEDs" in Chapter 3.

The images of different PG LTM-LEDs were taken by a scanning electron microscope (SEM) (JEOL JSM-5600LV) system. Micro-Raman spectra were also performed using a spectrometer (Horiba JY-T64000) equipped with an excitation laser of $532 \mathrm{~nm}$ wavelength to reveal the strain level. Electroluminescence (EL) spectra and the optical output power were acquired by an Ocean Optics spectrometer (QE65000) attached to an integrating sphere. The $1 \times 1 \mathrm{~mm}^{2}$ indium that uniformly covered on the p-GaN surface was used as current spreading layer. A LED tester (M2442S-9A Quatek Group) was used to measure the current-voltage characteristics of the LED chips.

\subsection{Experimental results of partitioned growth LTM-LEDs and discussion}

Figure 6.1 shows the SEM images for PG LTM-LEDs of different sizes. Figures 6.1(a), 6.1(c), 6.1(e), 6.1(g), and 6.1(h) show the top-view images for the $50 \times 50$ $\mu \mathrm{m}^{2}, 100 \times 100 \mu \mathrm{m}^{2}, 200 \times 200 \mu \mathrm{m}^{2}, 500 \times 500 \mu \mathrm{m}^{2}$, and $1,000 \times 1,000 \mu \mathrm{m}^{2} \mathrm{PG}$ LTM-LEDs, respectively. Figures 6.1(b), 6.1(d), and 6.1(f) show the $45^{\circ}$ tilted view images for the $50 \times 50 \mu \mathrm{m}^{2}, 100 \times 100 \mu \mathrm{m}^{2}$, and $200 \times 200 \mu \mathrm{m}^{2}$ PG LTM-LEDs, respectively. As the top-view and $45^{\circ}$ tilted view images for the $500 \times 500 \mu \mathrm{m}^{2}$ and 
$1,000 \times 1,000 \mu \mathrm{m}^{2}$ sizes look similar, here we only show the top-view images for these two sizes. From these images, we can observe that smaller PG LTM-LEDs have a larger ratio of opening area. Since the chip dimension for PG LTM-LEDs is smaller than the anode which is made of a $1 \times 1 \mathrm{~mm}^{2}$ indium contact on the $\mathrm{p}-\mathrm{GaN}$ surface, the active light emission area has to be taken into consideration. To facilitate the analysis, we use the square of the side length over the square of the side length plus half of the margin width $(10 \mu \mathrm{m})$ as the ratio of active lighting area. For example, the ratio of active lighting area for $50 \times 50 \mu \mathrm{m}^{2}$ device is $50 \times 50$ over $(50+5) \times(50+5)$. After calculation, we find that the ratios of active lighting area are $83 \%, 91 \%, 95 \%, 98 \%$, and $99 \%$, respectively, for the $50 \times 50 \mu \mathrm{m}^{2}, 100 \times 100 \mu \mathrm{m}^{2}$, $200 \times 200 \mu \mathrm{m}^{2}, 500 \times 500 \mu \mathrm{m}^{2}$, and $1,000 \times 1,000 \mu \mathrm{m}^{2}$ devices. From the calculation results, we can see that $50 \times 50 \mu \mathrm{m}^{2}$ PG LTM-LEDs have a much smaller ratio of the active light emission area than others. 


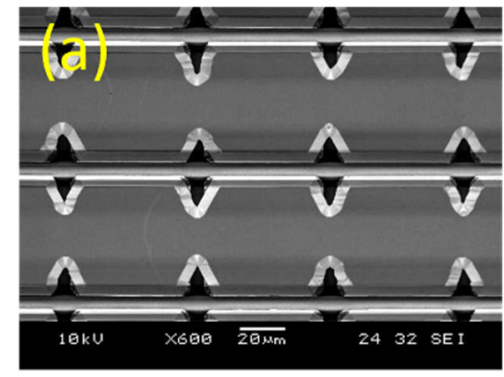

(a) $50 \mu \mathrm{m}$ top-view

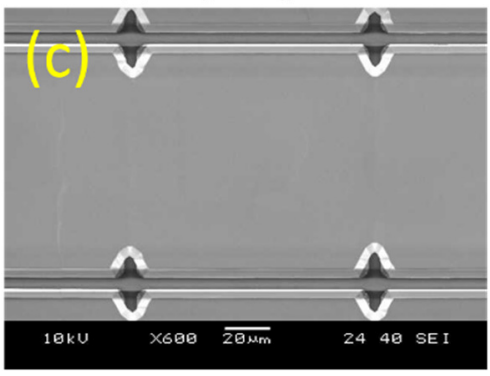

(c) $100 \mu \mathrm{m}$ top-view

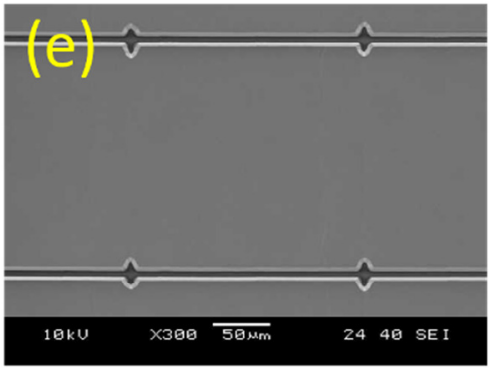

(e) $200 \mu \mathrm{m}$ top-view

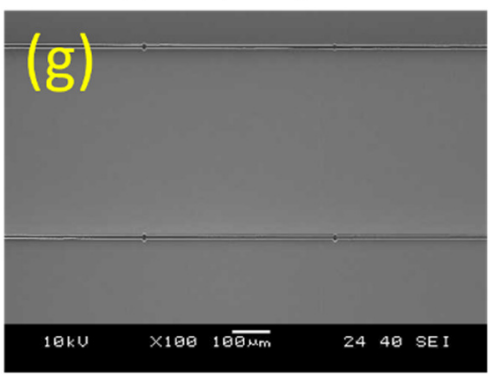

(g) $500 \mu \mathrm{m}$ top-view

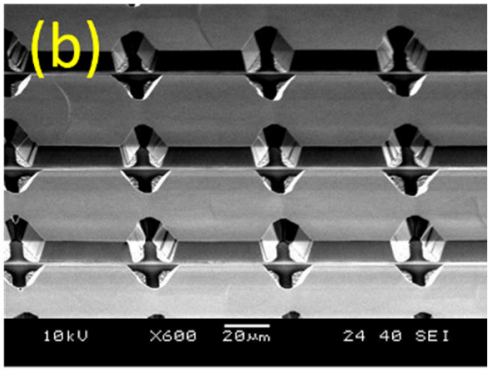

(b) $50 \mu \mathrm{m}$ tilt by $45^{\circ}$

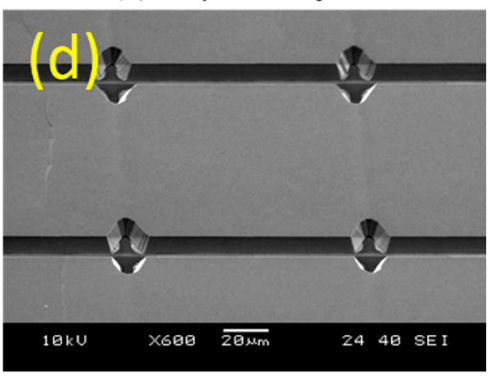

(d) $100 \mu \mathrm{m}$ tilt by $45^{\circ}$

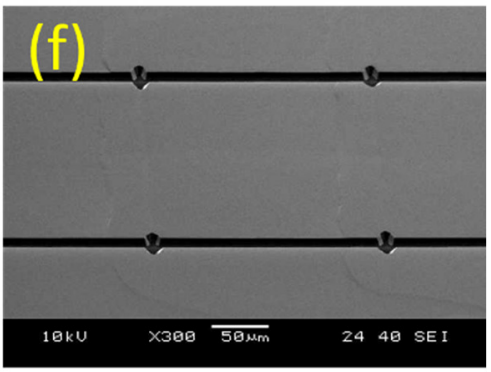

(f) $200 \mu \mathrm{m}$ tilt by $45^{\circ}$

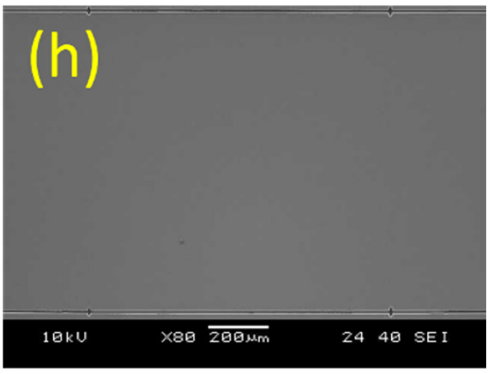

(h) $1000 \mu \mathrm{m}$ top-view

Figure 6.1 The SEM images of PG LTM-LEDs with different sizes: top-view images for (a) $50 \times 50 \mu \mathrm{m}^{2}$, (c) $100 \times 100 \mu \mathrm{m}^{2}$, (e) $200 \times$ $200 \mu \mathrm{m}^{2},(\mathrm{~g}) 500 \times 500 \mu \mathrm{m}^{2}$, and (h) $1,000 \times 1,000 \mu \mathrm{m}^{2}$ chips; $45^{\circ}$ 
tilted view images for (b) $50 \times 50 \mu \mathrm{m}^{2}$, (d) $100 \times 100 \mu \mathrm{m}^{2}$, and (f) $200 \times 200 \mu \mathrm{m}^{2}$ chips.

The optical output power and the external quantum efficiency (EQE) for PG LTM-LEDs of different sizes are depicted in Figures 6.2(a) and 6.2(b), which show that the optical performance is enhanced as the device size decreases from $1,000 \times$ $1,000 \mu \mathrm{m}^{2}$ to $100 \times 100 \mu \mathrm{m}^{2}$. Higher optical output power can be obtained in smaller devices at a given current. However, the $50 \times 50 \mu \mathrm{m}^{2}$ device shows a smaller optical output power and EQE than others at the lower current density. As we have mentioned previously, the $50 \times 50 \mu \mathrm{m}^{2}$ device has the smallest ratio of active lighting area, which may be one of the underlying reasons. Nevertheless, it seems that the small ratio of active lighting area is not the only reason for such a degradation for the $50 \times 50 \mu \mathrm{m}^{2}$ device. To figure out the underlying reasons of these phenomena, a series of characterization methods are performed and the results are analyzed. 

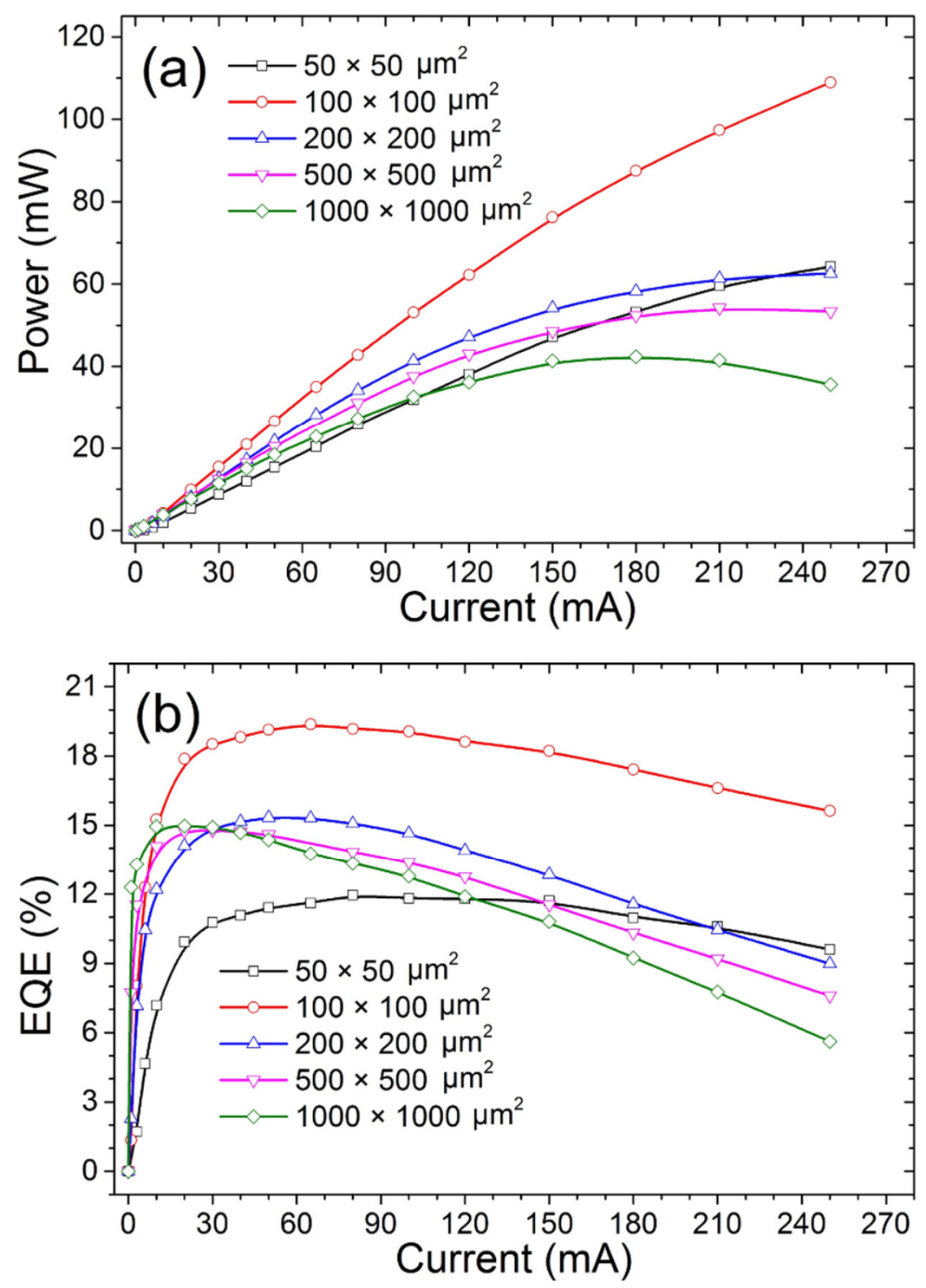

Figure 6.2 (a) The power versus current and (b) the EQE versus current of PG LTM-LEDs in different sizes.

Raman scattering, has been a widely used method to study the strain for IIInitrides [136]. As the $E_{2}$ (high) phonon frequency is sensitive to strain, it has been extensively applied to quantify the in-plane stress in $\mathrm{GaN}[137,138]$. It is widely accepted that the peak of $E_{2}$ (high) phonon in the unstrained $\mathrm{GaN}$ layer is $567.6 \mathrm{~cm}^{-}$ 
${ }^{1}$ [139], and the blueshift for the $E_{2}$ (high) phonon frequency indicates the in-plane compressive stress, while a redshift indicates the in-plane tensile stress. As depicted in Figure 6.3, Raman spectra of $E_{2}$ (high) phonon peaks for different PG LTM-LEDs show that all the measured $E_{2}$ (high) phonon frequencies of PG LTM-LEDs are blueshift, which accounts for the in-plane compressive stress. From Figure 6.3, we can also see that with the size decreasing from $1,000 \times 1,000 \mu \mathrm{m}^{2}$ to $50 \times 50 \mu \mathrm{m}^{2}$, the $E_{2}$ (high) phonon peaks move toward to the unstrained $567.6 \mathrm{~cm}^{-1}$, which indicates that the in-plane compressive stress is released with the size decreasing. To facilitate the analysis, the in-plane compressive stress is further calculated according to $[140]$

$$
\sigma=\frac{\Delta \omega}{4.3} \mathrm{~cm} \cdot G P a
$$

where $\sigma$ is the biaxial stress and $\Delta \omega$ is the $E_{2}$ phonon peak difference between the strained $\mathrm{GaN}$ and unstrained $\mathrm{GaN}\left(567.6 \mathrm{~cm}^{-1}\right)$. The calculated in-plane compressive stress values of different sized PG LTM-LEDs are shown in Table 6.1. From Table 6.1, it is clear that the in-plane stress reduces as the size decreases. The reduced in-plane compressive stress for the $\mathrm{GaN}$ template layer grown on the sapphire substrate correspondingly suppresses the QCSE level in the InGaN/GaN quantum wells. The reduced QCSE suggests that by decreasing the PG LTM-LED size the output power and EQE can be improved. However, as can be observed from Figure 6.2, when the chip size is below $100 \times 100 \mu \mathrm{m}^{2}$, the other effects need to be taken into account because the device performance decreases. 


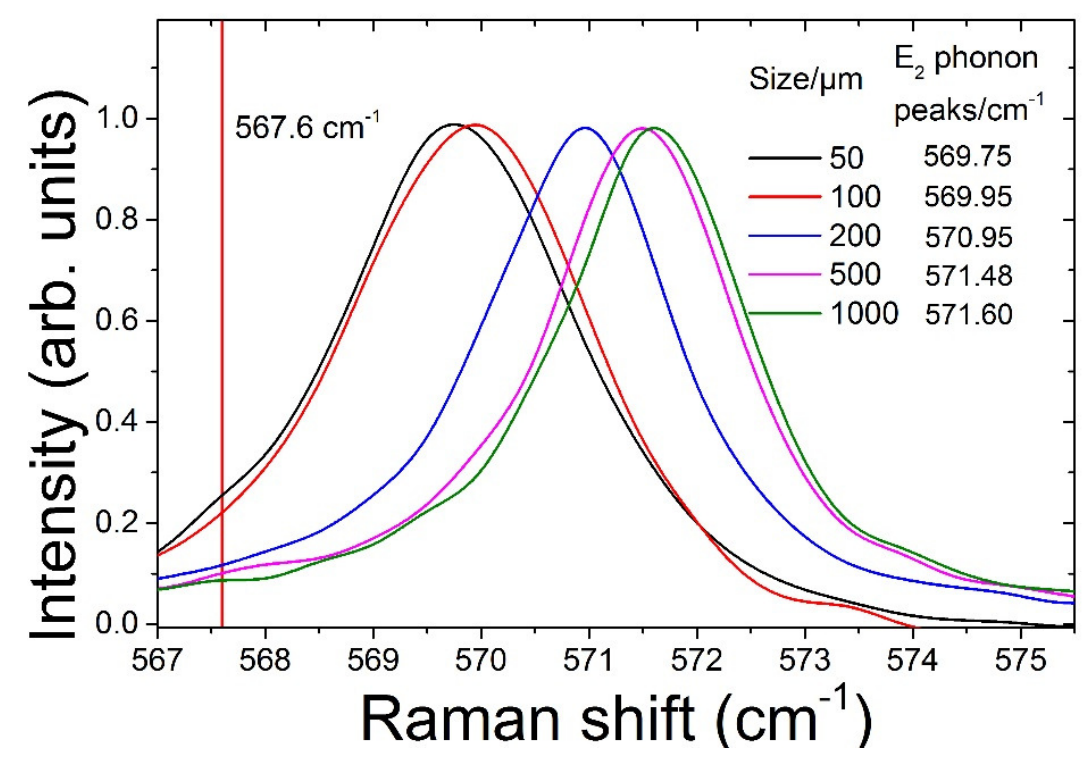

Figure 6.3 Raman spectrum of $E_{2}$ (high) phonon peaks for different sized PG LTM-LEDs. (Measured at the position $10 \mu \mathrm{m}$ off the edge)

Table 6.1 The in-plane compressive stress of different sized PG LTMLEDs.

\begin{tabular}{|c|c|c|c|c|c|}
\hline Size $(\boldsymbol{\mu m})$ & 50 & 100 & 200 & 500 & 1,000 \\
\hline Stress value $(\mathbf{G P a})$ & 0.50 & 0.55 & 0.78 & 0.90 & 0.93 \\
\hline
\end{tabular}

As the in-plane stress reduces, the blueshift for the wavelength caused by the reduced QCSE in the quantum wells is expected. The EL spectra for the studied PG LTM-LEDs at 20mA are depicted in Figure 6.4. As expected, the wavelength is blueshifted with the size decreasing from $1,000 \times 1,000 \mu \mathrm{m}^{2}$ to $200 \times 200 \mu \mathrm{m}^{2}$, as can be seen from Figure 6.4(a); however, the blueshift for the wavelength is very small. This phenomenon is because the blueshift for the wavelength caused by the reduced QCSE in the quantum wells can be partially compensated by the redshift, 
which is due to the more indium incorporation into the quantum wells with less stress $[141,142]$. On the other hand, the wavelength shows redshift as the size is further decreased from $200 \times 200 \mu \mathrm{m}^{2}$ to $50 \times 50 \mu \mathrm{m}^{2}$, as can be seen from Figure $6.4(\mathrm{~b})$, which is not expected. For the $50 \times 50 \mu \mathrm{m}^{2}$ and $100 \times 100 \mu \mathrm{m}^{2}$ cases, with the inplane stress further reducing, the effect of the indium incorporation is stronger than that of the QCSE suppression, so we see the wavelength redshift. However, this high concentration of indium not only makes the peak wavelength redshift, but also introduces more defects [142], which can be proved by the shift of EQE peak toward higher current [120], as shown in Figure 6.2(b). It has also been reported that point defects enhance the nonradiative recombination [143], which can be another reason for the lower output power and EQE for the $50 \times 50 \mu \mathrm{m}^{2}$ size when the current is smaller than $120 \mathrm{~mA}$. 

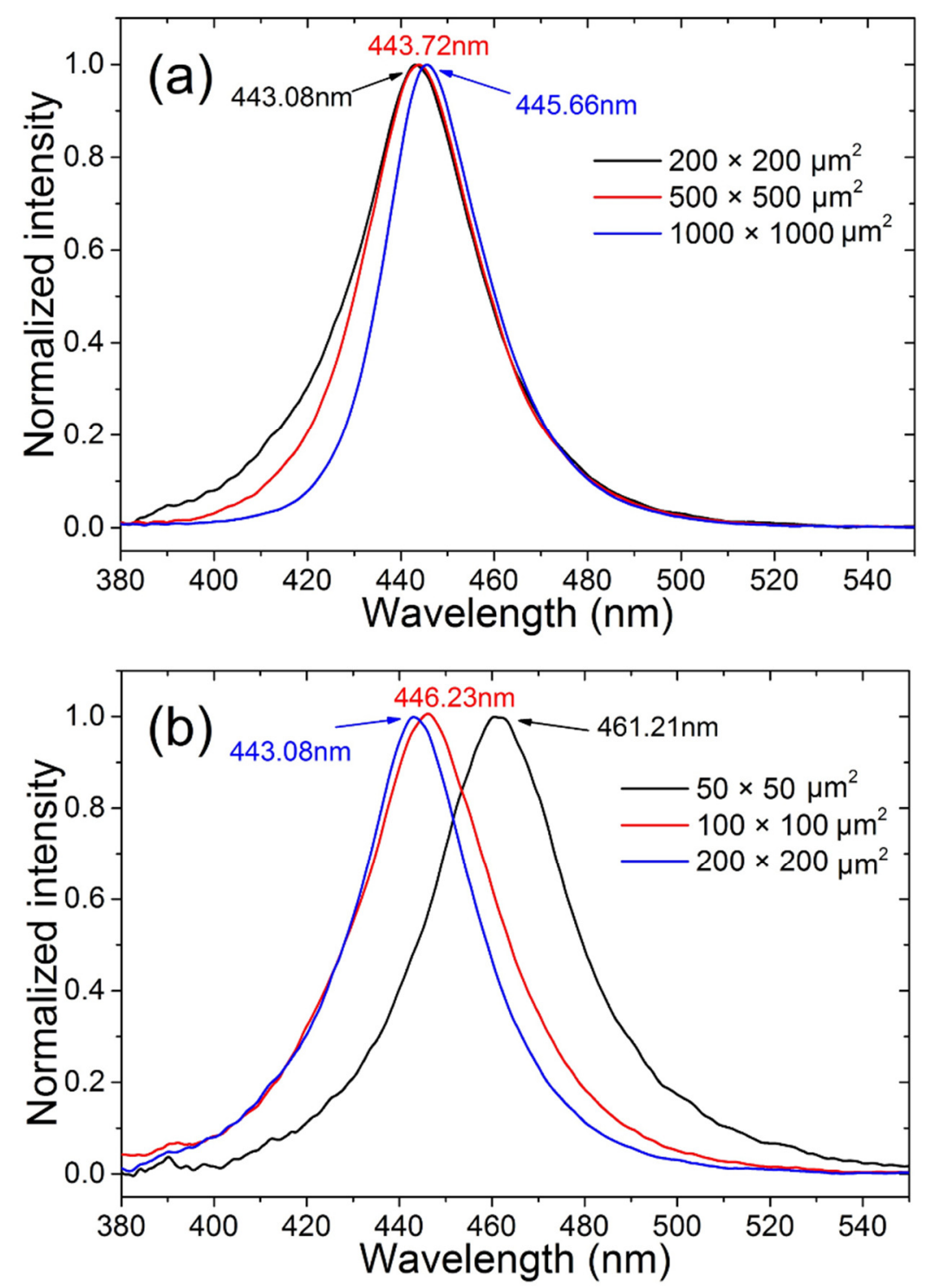

Figure 6.4 EL spectra of PG LTM-LEDs of different sizes at 20mA:

(a) the $1,000 \times 1,000 \mu \mathrm{m}^{2}, 500 \times 500 \mu \mathrm{m}^{2}$, and $200 \times 200 \mu \mathrm{m}^{2}$ devices; (b) the $200 \times 200 \mu \mathrm{m}^{2}, 100 \times 100 \mu \mathrm{m}^{2}$, and $50 \times 50 \mu \mathrm{m}^{2}$ devices.

We further carry out the current voltage (I-V) characteristics for PG LTM-LEDs of different sizes in Figure 6.5. From Figure 6.5 we can see that the reverse leakage 
current increases when the PG LTM-LED size is decreased, indicating more defects in the quantum wells [143]. It is worth noting that the $50 \times 50 \mu \mathrm{m}^{2}$ device shows the largest leakage current when the device is reversely biased, which is a signature of the high defect density. In our case, the higher defect density in the quantum wells is ascribed to the higher indium incorporation efficiency for the smaller PG LTMLEDs, and therefore, further epi-growth optimizations are required.

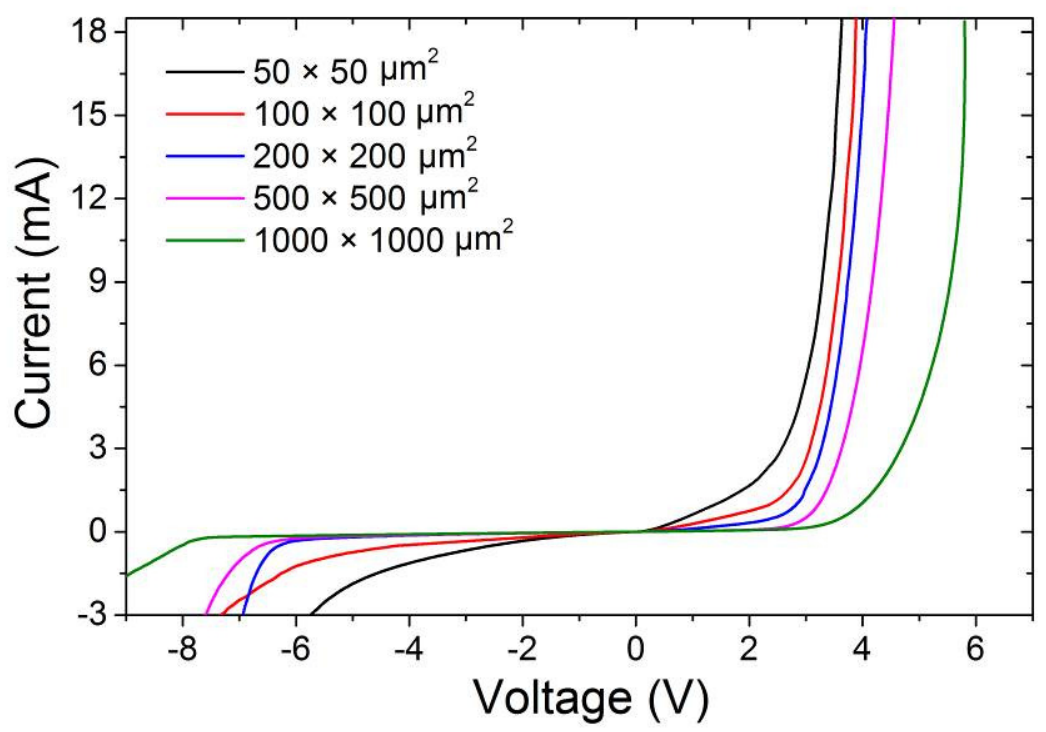

Figure 6.5 I-V characteristics for PG LTM-LEDs of different sizes.

\subsection{Conclusion}

In this work, to study the size effect on strain release, LTM-LEDs in $50 \times 50$ $\mu \mathrm{m}^{2}, 100 \times 100 \mu \mathrm{m}^{2}, 200 \times 200 \mu \mathrm{m}^{2}, 500 \times 500 \mu \mathrm{m}^{2}$, and $1,000 \times 1,000 \mu \mathrm{m}^{2}$ sizes were grown on patterned c-plane sapphire substrate by the MOCVD technique. Various characterization methods are performed to study the strain for different PG 
LTM-LEDs. The output power and EQE characteristics show that the optical performance can be enhanced when the PG LTM-LED size is reduced from 1,000 $\times$ $1,000 \mu \mathrm{m}^{2}$ to $100 \times 100 \mu \mathrm{m}^{2}$. The improved EQE is due to the reduced QCSE in the InGaN/GaN quantum wells for those PG LTM-LEDs. However, for the $50 \times 50 \mu \mathrm{m}^{2}$ size, the optical performance is limited by the smallest effective lighting area and more defects induced by higher indium incorporation. Therefore, in our case, the 100 $\times 100 \mu \mathrm{m}^{2}$ size delivers the highest output power and EQE. In summary, our experimental results indicate that to get high quality epitaxy based on partitioned growth, a small size is needed given that the defects density and margin ratio is carefully controlled. Since this partitioned growth method is easy and low-cost to realize based on the current commercial sapphire substrate LED growth process, PG LTM-LEDs will significantly speed up the LED applications in our daily life. 


\section{Chapter 7. Conclusions and Recommendations}

\subsection{Conclusions}

The challenges for LEDs used in special applications have been reviewed. High quality epitaxial LED wafers have been grown using MOCVD systems. Optimized LED fabrication techniques for flip-chip and vertical LEDs are demonstrated. Based on these growth and fabrication techniques, LTM-LEDs are designed and fabricated to offer an optimized output power performance for special applications such as communications and micro-displays.

In chapter 4, we discussed the size effect and the fabrication limitation of LTMLEDs. An optimal mesa size for the current density and power density performance of the LTM-LEDs based on experimental and numerical results is found and demonstrated. Furthermore, we conclude that as a design rule of thumb for high current density and high power density LTM-LEDs, three key points were listed: first, the current spreading layer size should be made as close as possible to the mesa size; second, an optimum mesa size should be determined according to the specific layer structures and the design layout; third, the n-pad should be made as close to the p-pad as possible before any severe current leakage happens.

Chapter 5 further investigated the shape effect on the electrical and optical properties of LTM-LEDs, and new structured triangle-, circle-, and square-shaped LTM-LEDs were designed and realized in the flip-chip configuration. It was revealed that the triangle-shaped LTM-LEDs deliver the highest current density 
against the bias and the highest power density against the current density among all devices. The underlying reason is that the triangle-shaped LTM-LEDs have a lower resistance at the mesa edge and a shorter $\mathrm{n}-\mathrm{GaN}$ current path. The lower resistance at the mesa edge and the shorter current path not only reduces Joule heat but also contributes to uniform current density, which makes triangle-shaped LTM-LEDs to have a higher average radiative recombination rate. This is totally a novel discovery compared with the previously reported study stating that side-wall light extraction contributes to higher power of the triangular LEDs.

Finally, in chapter 6, we studied the size effect of strain release on LTM-LEDs in different sizes. These LTM-LEDs were grown on patterned c-plane sapphire substrate by the MOCVD technique. The output power and EQE characteristics show that the optical performance can be enhanced when the PG LTM-LED size is reduced from $1,000 \times 1,000 \mu \mathrm{m}^{2}$ to $100 \times 100 \mu \mathrm{m}^{2}$. The improved EQE is due to the reduced QCSE in the InGaN/GaN quantum wells for those small PG LTM-LEDs. However, when the size further reduces to $50 \times 50 \mu \mathrm{m}^{2}$, the optical performance is limited by the small effective lighting area and the defects induced by higher indium incorporation. Therefore, in our case, the $100 \times 100 \mu \mathrm{m}^{2}$ size delivers the highest output power and EQE.

In summary, this dissertation covers the knowledge of epitaxial growth, LED chip design, device fabrication, characterization, physical modeling, and numerical simulation. The proposed devices are studied both from experiment and simulation. Optimal shape and size of LTM-LEDs for highest performance are found based on our growth and fabrication conditions, and the underlying physics are clearly 
understood based on our physical model and numerical simulation. This thesis work affords an insightful understanding on LTM-LEDs and provides a good rule of thumb on how to get high current density, high power density, and high response speed LTM-LEDs with optimal performance.

\subsection{Recommendations for future work}

As the LTM-LEDs performance is continuing to improve, increasingly more diverse applications are being found for them. High brightness and high response speed are two of the most important characteristics for many new applications such as LED communications and micro-displays. To further optimize the performance of LTM-LEDs, several aspects are recommended for future work to make LTMLEDs brighter and faster.

\subsubsection{Patterned substrate growth for super high luminous efficacy}

Previously, partitioned growth LTM-LEDs have been studied and demonstrate that making the grown size smaller is an effective way to improve the electrical and optical performance. To further improve the LTM-LEDs, we suggest to apply a nano-patterned sapphire substrate, as shown in Figure 7.1.

\section{(a)}

(b)

Figure 7.1 Schematic diagram of (a) planer sapphire substrate and (b) patterned sapphire substrate. 
Patterned sapphire substrate (PSS) has been commonly used both in research institute and industry. There are two main ways for PSS to improve the LED power. One is to improve the crystalline quality through releasing the stress attributed to the lattice mismatch. The other one is to increase the LEE by diffusing the light to restrain the total internal reflection. Recently, the nano-patterned sapphire substrate has been intensively studied and hence efficiency of LEDs has been further enhanced $[144,145]$. However, the strain in the partitioned growth LTM-LEDs is still strong and the QCSE needs to be further alleviated. Hence, to address these issues, we suggest to deposit partitioned growth LTM-LEDs on nano-patterned sapphire substrate with optimized nano-pattern periods and pattern shapes to further release the strain. This can be done by the nanoimprinting technique which can be used to make the nano-patterned sapphire substrate. The characteristics of the partitioned growth LTM-LED epitaxial layer can be measured by micro-Raman spectroscopy and transmission electron microscope (TEM) analysis, and the surface topography can be examined by SEM and atomic force microscope (AFM).

\subsubsection{Vertical high-speed LTM-LEDs}

To increase the maximum modulation frequency of LEDs, one of the common approaches is to shorten the minority carrier lifetime. There are two ways to realize it; one is doping the active region with a high density of dopants and the other one is to deliberately introduce deep traps. However, these two approaches reduce the EQE of the LED and generate more heat [146]. 


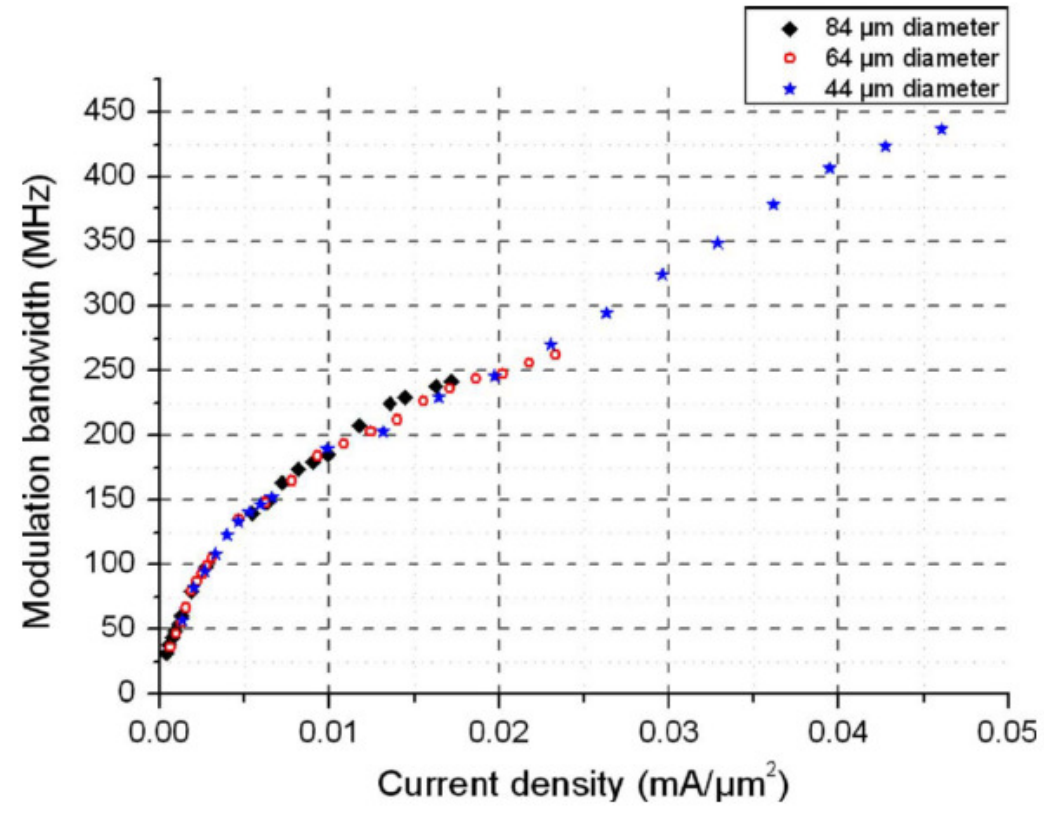

Figure 7.2 Bandwidth versus current density graph for the $450 \mathrm{~nm}-$ emitting LEDs [33].

From the literature we find that the bandwidth can be increased with the current density increasing as the differential carrier lifetime is shorter at higher current densities [33]. Figure 7.2 shows the bandwidth versus current density. Thus, it is desirable to increase the current density and at the same time keep or increase the power density as a better solution for high-speed LEDs. Hence, I am numerically investigating the vertical LTM-LEDs as a possible solution for a high-speed LED. Figure 7.3 shows that the vertical LEDs have much higher current density, better IQE, and power density than flip-chip LEDs which is adopted as the structure for our LTM-LEDs. The reason for this behaviour is because the vertical LTM-LEDs has a more uniform current density and a shorter n-GaN current route compared with the flip-chip LEDs, as shown in Figure 7.4. Thus, the vertical LTM-LEDs have the 
potential to be the ideal solution for the high-speed LTM-LEDs.
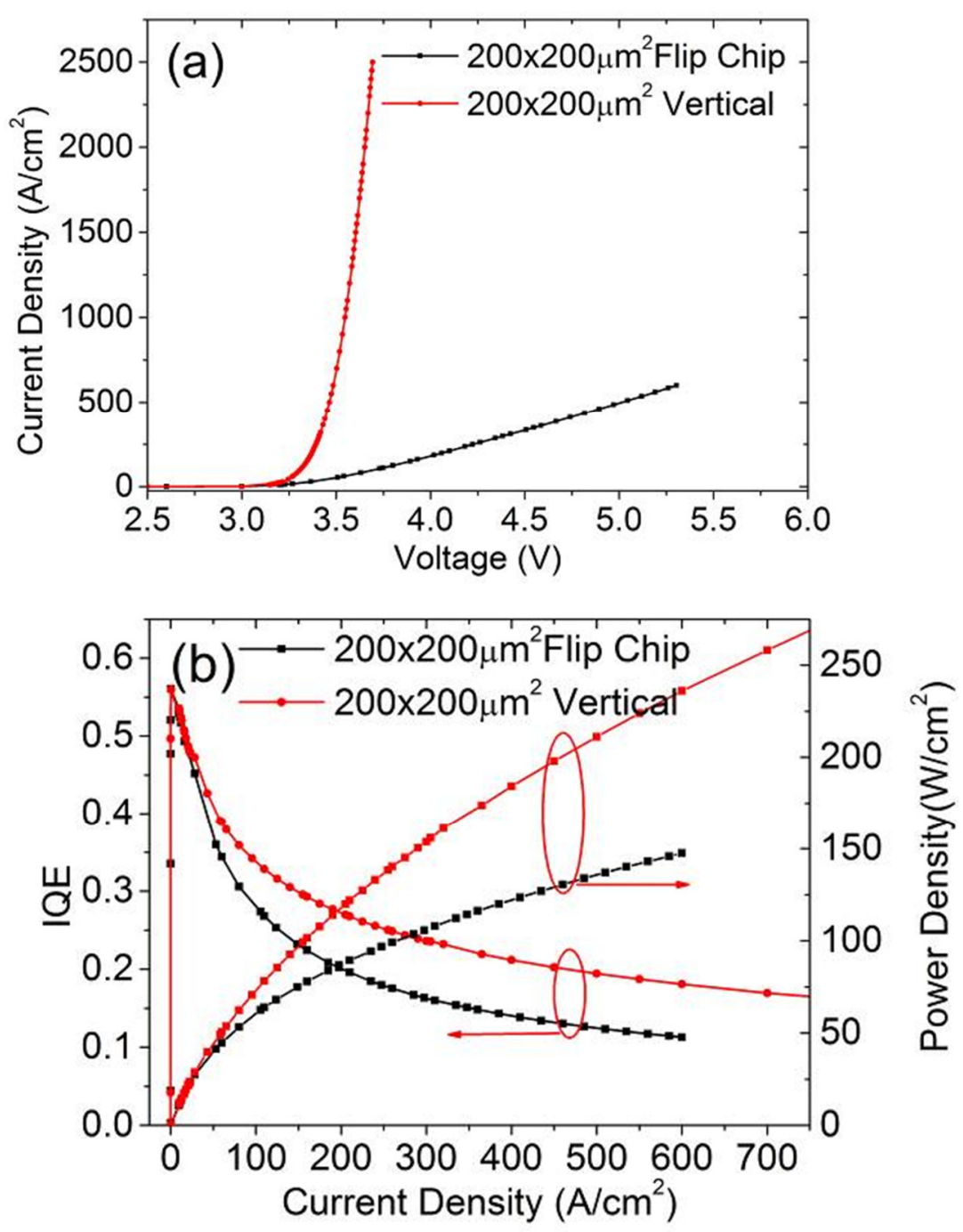

Figure 7.3 Simulated results of (a) $J-V$ characteristics of and (b) $I Q E \& P D-J$ characteristics of flip-chip versus vertical LTM-LEDs where the $\mathrm{p}-\mathrm{GaN}$ is fully covered with metal and heat model is considered. 
(a)
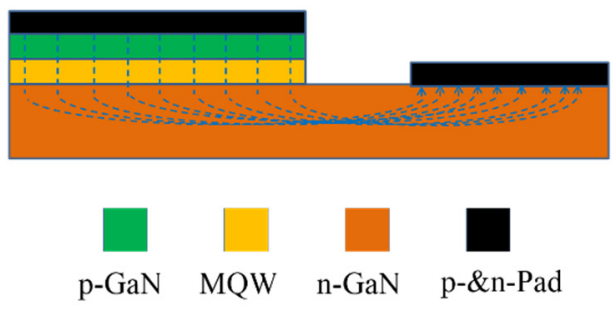

(b)

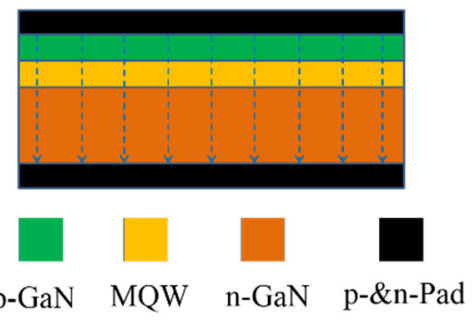

Figure 7.4 The schematic current paths of (a) flip-chip LEDs; and (b) vertical LEDs.

\subsubsection{Regular patterned n-GaN surface to increase the light extraction of vertical LTM-LEDs}

For vertical LTM-LEDs, as the double side polished sapphire substrate is peeling off and the surface is flat, part of the light generated by the active area is confined in the LED owing to total internal reflection. Random pattern on $\mathrm{n}-\mathrm{GaN}$ has been tried on our vertical broad-area LEDs as a solution for this issue. This random pattern can increase the light output power by about 5-10\% based on our experimental data. In the future, we recommend to further study on the regular patterned n-GaN surface to improve the output power of vertical LTM-LEDs. Figure 7.5(b) shows the regular patterned $n-G a N$ surface. Compared to the flat n-GaN surface, which will have total internal reflection, as shown in Figure 7.5(a), regular patterned n-GaN surface will diffuse the light into a random direction and then increase the light extraction. 

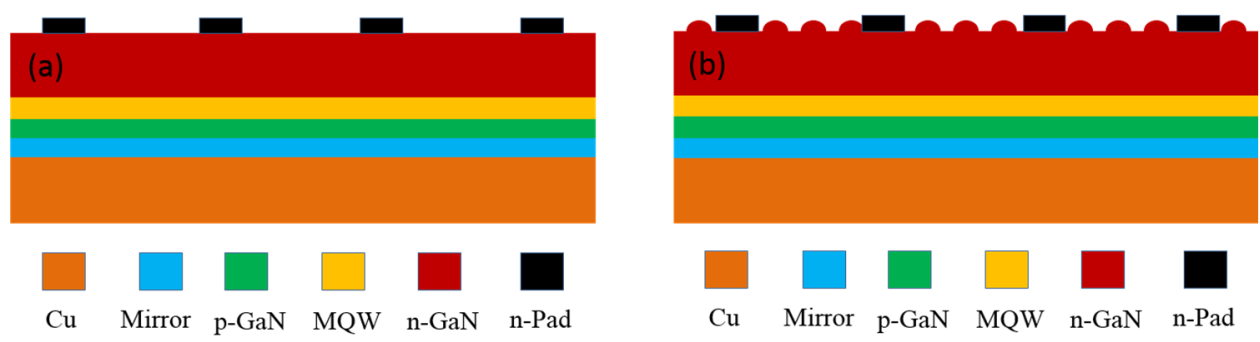

Figure 7.5 Vertical LEDs with (a) flat n-GaN surface and (b) regular patterned n-GaN surface. 


\section{Publication List}

1. S. Lu, W. Liu, Z.-H. Zhang, S.T. Tan, Z. Ju, Y. Ji, X. Zhang, Y. Zhang, B. Zhu, and Z. Kyaw, N. Hasanov, X. W. Sun, and H. V. Demir, "Low thermal-mass LEDs: size effect and limits," Optics Express, 2014. 22(26): p. 32200-32207.

2. S. Lu, S.T. Tan, B. Zhu, Y. Zhang, and N. Hasanov, and H. V. Demir, “Triangular, circular and square micro-light-emitting diodes for high current density and high power density applications: shape effect in micro- light-emitting diodes," in preparation.

3. S. Lu, Z.-H. Zhang, S.T. Tan, B. Zhu, Y. Zhang, and N. Hasanov, and H. V. Demir, "Strain-reduced micro-light-emitting diodes directly grown using partitioned growth," in preparation.

4. Binbin Zhu, Swee Tiam Tan, Wei Liu, Shunpeng Lu, Yiping Zhang, Shi Chen, Namig Hasanov, Xuejun Kang, and Hilmi Volkan Demir, "Modulating ohmic contact through InGaxNyOz interfacial layer for high-performance InGaN/GaNbased light-emitting diodes”, Photonics Journal, IEEE, 2016. 8(3):1600808.

5. Binbin Zhu, Wei Liu, Shunpeng Lu, Yiping Zhang, Namig Hasanov, Xueliang Zhang, Yun Ji, Zi-Hui Zhang, Swee Tiam Tan, Hongfei Liu, and Hilmi Volkan Demir, "Decoupling contact and mirror: an effective way to improve the reflector for flip-chip InGaN/GaN-based light-emitting diodes”, Journal of Physics D: Applied Physics, 2016. 49: 265106.

6. Hasanov, N., B. Zhu, V.K. Sharma, S. Lu, Y. Zhang, W. Liu, S.T. Tan, X.W. Sun, and H.V. Demir, "Improved performance of InGaN/GaN flip-chip light- 
emitting diodes through the use of robust Ni/Ag/TiW mirror contacts," Journal of Vacuum Science \& Technology B, 2016. 34(1): p. 011209.

7. Zhang, Z.-H., W. Liu, S.T. Tan, Z. Ju, Y. Ji, Z. Kyaw, X. Zhang, N. Hasanov, B. Zhu, and S. Lu, Y.P. Zhang, X. W. Sun, and H. V. Demir, "On the mechanisms of InGaN electron cooler in InGaN/GaN light-emitting diodes," Optics Express, 2014. 22(103): p. A779-A789.

8. Zhang, Z.-H., W. Liu, S.T. Tan, Y. Ji, L. Wang, B. Zhu, Y. Zhang, S. Lu, X. Zhang, and N. Hasanov, X.W. Sun, and H.V. Demir, "A hole accelerator for InGaN/GaN light-emitting diodes," Applied Physics Letters, 2014. 105(15): p. 153503.

9. Zhang, Z.-H., Y. Ji, W. Liu, S.T. Tan, Z. Kyaw, Z. Ju, X. Zhang, N. Hasanov, S. Lu, and Y. Zhang, B. Zhu, X.W. Sun, and H.V. Demir, "On the origin of the electron blocking effect by an n-type AlGaN electron blocking layer," Applied Physics Letters, 2014. 104(7): p. 073511.

10. Zhang, Y.P., Z.-H. Zhang, W. Liu, S.T. Tan, Z.G. Ju, X.L. Zhang, Y. Ji, L.C. Wang, Z. Kyaw, and N. Hasanov, B. Zhu, S. Lu, X. W. Sun, and H. V. Demir, "Nonradiative recombination—critical in choosing quantum well number for InGaN/GaN light-emitting diodes," Optics Express, 2015. 23(3): p. A34-A42.

11. Kyaw, Z., Z.-H. Zhang, W. Liu, S.T. Tan, Z.G. Ju, X.L. Zhang, Y. Ji, N. Hasanov, B. Zhu, and S. Lu, Y.P. Zhang, X. W. Sun, and H. V. Demir, " On the effect of N-GaN/P-GaN/N-GaN/P-GaN/N-GaN built-in junctions in the $n-G a N$ layer for InGaN/GaN light-emitting diodes," Optics Express, 2014. 22(1): p. 809-816. 
12. Kyaw, Z., Z.-H. Zhang, W. Liu, S.T. Tan, Z.G. Ju, X.L. Zhang, Y. Ji, N. Hasanov, B. Zhu, and S. Lu, Y.P. Zhang, J. H. Teng, X. W. Sun, and H. V. Demir, "Simultaneous enhancement of electron overflow reduction and hole injection promotion by tailoring the last quantum barrier in InGaN/GaN lightemitting diodes," Applied Physics Letters, 2014. 104(16): p. 161113.

13. Ju, Z.G., W. Liu, Z.-H. Zhang, S.T. Tan, Y. Ji, Z. Kyaw, X.L. Zhang, S.P. Lu, Y.P. Zhang, and B.B. Zhu, N. Hasanov, X. W. Sun, and H. V. Demir, "Advantages of the Blue InGaN/GaN Light-Emitting Diodes with an AlGaN/GaN/AlGaN Quantum Well Structured Electron Blocking Layer,” ACS Photonics, 2014. 1(4): p. 377-381.

14. Ju, Z., W. Liu, Z.-H. Zhang, S.T. Tan, Y. Ji, Z. Kyaw, X. Zhang, S. Lu, Y. Zhang, and B. Zhu, N. Hasanov, X. W. Sun, and H. V. Demir, "Improved hole distribution in InGaN/GaN light-emitting diodes with graded thickness quantum barriers," Applied Physics Letters, 2013. 102(24): p. 243504.

15. Y. Zhang, Z.-H. Zhang, S. T. Tan, P. L. Hernandez-Martinez, B. Zhu, S. Lu, X. J. Kang, X. W. Sun, and H. V. Demir, "Investigation of p-type depletion doping for InGaN/GaN-based light-emitting diodes," Applied Physics Letters, 2017. 110(3): p. 033506. 


\section{Bibliography}

1. A. Bergh, G. Craford, A. Duggal, and R. Haitz, "The promise and challenge of solid-state lighting," Phys Today 54, 42-47 (2001).

2. G. Roos, "Cree Breaks 300 Lumens-Per-Watt Barrier," EPSNews https://epsnews.com/2014/03/26/cree-reaches-led-milestone/, 2014 (Accessed in April 2016).

3. C. Gupta, "Energy \& Maintenance Savings with LED Lighting," SlideShare http://www.slideshare.net/ChristineGupta/energy-maintenance-savings-withled-lighting, 2013 (Accesssed in April 2016).

4. J. Edmond, "Reinventing Lighting," DOE SSL R\&D Workshop, San Francisco, CA, http://www.energy.gov/sites/prod/files/2015/2002/f2019/edmond reinventing _sanfrancisco2015.pdf, 2015 (Accessed May 2015).

5. D. S. Program, "Life-Cycle Assessment of Energy and Environmental Impacts of LED Lighting Products," http://apps1.eere.energy.gov/buildings/publications/pdfs/ssl/lca factsheet apr 2013.pdf, 2013 (Accessed May 2015).

6. J. Y. Tsao, H. D. Saunders, J. R. Creighton, M. E. Coltrin, and J. A. Simmons, "Solid-state lighting: an energy-economics perspective," J Phys D Appl Phys 43, 354001 (2010).

7. R. Haitz, "Haitz's law," Nat Photonics 1, 23-23 (2007).

8. Z. Liu, S. Liu, K. Wang, and X. Luo, "Status and prospects for phosphor-based white LED packaging," Frontiers of Optoelectronics in China 2, 119-140 (2009).

9. T. Whitaker, "Cree reports $R \& D$ result of $231 \mathrm{~lm} / \mathrm{W}$ efficacy for white LED," LEDs Magazine http://ledsmagazine.com/news/8/5/8, 2011 (Accessed in April 2016).

10. M. Wright, "Cree announces a new laboratory LED efficacy milestone at SIL," LEDs Magazine http://ledsmagazine.com/news/8/5/8, 2013 (Accessed in April 2016).

11. SemLight, "LED street lights on FDR Drive in midtown Manhattan, 2011," http://www.semlight.com/Solar-LED-Street-Light-Projects/Solar-LED-StreetLight-Projects-145.html, 2011 (Accessed in April 2016). 
12. H. Lighting, "LED swimming pool lights application," http://www.szhxgh.com/projectEinfo_301_307.html, 2010 (Accessed in April 2016).

13. D. Wagen, "Chic and Eco Friendly Commercial Store Interior Design of Eco Yogurt Lounge, Boca Raton," http://www.designwagen.com/article/14749, 2015 (Accessed in April 2016).

14. Wikipedia, "Daytime running lamp," https://en.wikipedia.org/wiki/Daytime_running_lamp, 2016 (Accessed in April 2016).

15. AliExpress, "China factory coral reef used 90W LED plant growth lighting with dimming program," http://www.aliexpress.com/store/product/Chinafactory-coral-reef-used-90W-LED-plant-growth-lighting-with-dimmingprogram/1848623_32404116040.html, 2015 (Accessed in April 2016).

16. S. b. Williams, "The Future is Here: The Li-Fi Network," https://storiesbywilliams.com/2013/05/03/the-future-is-here-the-li-finetwork/, 2013 (Accessed in April 2016).

17. E. F. Schubert, and J. K. Kim, "Solid-state light sources getting smart," Science 308, 1274-1278 (2005).

18. L. H. Koh, Y. K. Tan, Z. Z. Wang, and K. J. Tseng, "An energy-efficient low voltage DC grid powered smart LED lighting system," in IECON 2011 - 37th Annual Conference on IEEE Industrial Electronics Society, pp. 2883-2888 (2011).

19. A. Suzdalenko, and I. Galkin, "Choice of power and control hardware for smart LED luminary," in 2010 12th Biennial Baltic Electronics Conference, pp. 331334 (2010).

20. S. Muthu, F. J. P. Schuurmans, and M. D. Pashley, "Red, green, and blue LEDs for white light illumination," IEEE J Sel Top Quant 8, 333-338 (2002).

21. J. J. D. McKendry, R. P. Green, A. E. Kelly, G. Zheng, B. Guilhabert, D. Massoubre, E. Gu, and M. D. Dawson, "High-Speed Visible Light Communications Using Individual Pixels in a Micro Light-Emitting Diode Array," Photonics Technology Letters, IEEE 22, 1346-1348 (2010).

22. Cisco, "Cisco Visual Networking Index: Global Mobile Data Traffic Forecast Update, 2014-2019," http://www.cisco.com/c/en/us/solutions/collateral/service-provider/visualnetworking-indexvni/white_paper_c11-520862.pdf, 2015 (Accessed May 
2015).

23. H. X. Jiang, S. X. Jin, J. Li, J. Shakya, and J. Y. Lin, "III-nitride blue microdisplays," Appl Phys Lett 78, 1303-1305 (2001).

24. J. Day, J. Li, D. Y. C. Lie, C. Bradford, J. Y. Lin, and H. X. Jiang, "III-Nitride full-scale high-resolution microdisplays," Appl Phys Lett 99, 031116 (2011).

25. J. Day, J. Li, D. Y. C. Lie, C. Bradford, J. Y. Lin, and H. X. Jiang, "Full-scale self-emissive blue and green microdisplays based on GaN micro-LED arrays," Quantum Sensing and Nanophotonic Devices 6, 82681X (2012).

26. B. Xue, H. Yang, F. Yu, X. Wang, L. Liu, Y. Pei, P. Lu, H. Xie, Q. Kong, J. Li, X. Yi, J. Wang, and J. Li, "Colour tuneable micro-display based on LED matrix," Optoelectronic Devices and Integration 5, 92701B (2014).

27. M. Akhter, J. S. Lee, P. P. Maaskant, M. Rensing, N. Nudds, P. O. Brien, P. Degenaar, and B. Corbett, "A LED micro-display with 90 ??90 pixels on a 80 ??m pitch," in 2015 European Microelectronics Packaging Conference (EMPC), pp. 1-5 (2015).

28. H. W. Choi, M. D. Dawson, P. R. Edwards, and R. W. Martin, "High extraction efficiency InGaN micro-ring light-emitting diodes," Appl Phys Lett 83, 44834485 (2003).

29. S. X. Jin, J. Shakya, J. Y. Lin, and H. X. Jiang, "Size dependence of III-nitride microdisk light-emitting diode characteristics," Appl Phys Lett 78, 3532-3534 (2001).

30. Z. Gong, H. X. Zhang, E. Gu, C. Griffin, M. D. Dawson, V. Poher, G. Kennedy, P. M. W. French, and M. A. A. Neil, "Matrix-Addressable Micropixellated InGaN Light-Emitting Diodes With Uniform Emission and Increased Light Output," IEEE Transactions on Electron Devices 54, 2650-2658 (2007).

31. Z. Gong, E. Gu, S. R. Jin, D. Massoubre, B. Guilhabert, H. X. Zhang, M. D. Dawson, V. Poher, G. T. Kennedy, P. M. W. French, and M. A. A. Neil, "Efficient flip-chip InGaN micro-pixellated light-emitting diode arrays: Promising candidates for micro-displays and colour conversion," J Phys D Appl Phys 41, 094002 (2008).

32. H. W. Choi, C. W. Jeon, and M. D. Dawson, "High-resolution 128 x 96 nitride microdisplay," IEEE Electr Device L 25, 277-279 (2004).

33. J. J. D. McKendry, D. Massoubre, S. L. Zhang, B. R. Rae, R. P. Green, E. Gu, R. K. Henderson, A. E. Kelly, and M. D. Dawson, "Visible-Light Communications Using a CMOS-Controlled Micro-Light-Emitting-Diode 
Array," J Lightwave Technol 30, 61-67 (2012).

34. Z. Gong, S. Jin, Y. Chen, J. McKendry, D. Massoubre, I. M. Watson, E. Gu, and M. D. Dawson, "Size-dependent light output, spectral shift, and selfheating of $400 \mathrm{~nm}$ InGaN light-emitting diodes," Journal of Applied Physics 107, 013103 (2010).

35. R. N. Hall, G. E. Fenner, J. D. Kingsley, T. J. Soltys, and R. O. Carlson, "Coherent Light Emission From GaAs Junctions," Physical Review Letters 9, 366-368 (1962).

36. R. M. Potter, J. M. Blank, and A. Addamiano, "Silicon Carbide Light-Emitting Diodes," Journal of Applied Physics 40, 2253-2257 (1969).

37. S. Nakamura, "GaN Growth Using GaN Buffer Layer," Japanese Journal of Applied Physics 30, L1705 (1991).

38. S. Nakamura, T. Mukai, and M. Senoh, "Candela-class high-brightness InGaN/AlGaN double-heterostructure blue-light-emitting diodes," Appl Phys Lett 64, 1687-1689 (1994).

39. H. Sato, A. Tyagi, H. Zhong, N. Fellows, R. B. Chung, M. Saito, K. Fujito, J. S. Speck, S. P. DenBaars, and S. Nakamura, "High power and high efficiency green light emitting diode on free-standing semipolar (112 2 ) bulk GaN substrate," physica status solidi (RRL) - Rapid Research Letters 1, 162-164 (2007).

40. M. H. Crawford, "LEDs for Solid-State Lighting: Performance Challenges and Recent Advances," IEEE J Sel Top Quant 15, 1028-1040 (2009).

41. R. Haitz, and J. Y. Tsao, "Solid-state lighting: 'The case' 10 years after and future prospects," physica status solidi (a) 208, 17-29 (2011).

42. I. L. Azevedo, M. G. Morgan, and F. Morgan, "The Transition to Solid-State Lighting," P IEEE 97, 481-510 (2009).

43. J. Pandey, Y. T. Liao, A. Lingley, R. Mirjalili, B. Parviz, and B. P. Otis, "A Fully Integrated RF-Powered Contact Lens With a Single Element Display," IEEE T Biomed Circ S 4, 454-461 (2010).

44. V. Poher, N. Grossman, G. T. Kennedy, K. Nikolic, H. X. Zhang, Z. Gong, E. M. Drakakis, E. Gu, M. D. Dawson, P. M. W. French, P. Degenaar, and M. A. A. Neil, "Micro-LED arrays: a tool for two-dimensional neuron stimulation," J Phys D Appl Phys 41, 094014 (2008).

45. H. X. Jiang, and J. Y. Lin, "Nitride micro-LEDs and beyond - a decade progress review," Opt Express 21, A475-A484 (2013). 
46. H. J. Round, "A Note on Carborundum," Electr. World 49, 308 (1907).

47. N. Zheludev, "The life and times of the LED [mdash] a 100-year history," Nat Photon 1, 189-192 (2007).

48. M. I. Nathan, W. P. Dumke, G. Burns, F. H. Dill, and G. Lasher, "Stimulated emission of radiation from GaAs p-n junctions," Appl Phys Lett 1, 62-64 (1962).

49. J. Pankove, and M. Massoulie, "Injection luminescence from GaAs," Bull. Am. Phys. Soc 7, 88 (1962).

50. T. M. Quist, R. H. Rediker, R. Keyes, W. Krag, B. Lax, A. L. McWhorter, and H. Zeigler, "Semiconductor maser of GaAs," Appl Phys Lett 1, 91-92 (1962).

51. J. I. Pankove, and J. E. Berkeyheiser, "A light source modulated at microwave frequencies," IRE Transactions on Electron Devices 9, 503-503 (1962).

52. S. Nakamura, N. Iwasa, and M. Senoh, "Method of manufacturing P-type compound semiconductor," United States Patent, US 5306662 A (1994).

53. I. Akasaki, "Key inventions in the history of nitride-based blue LED and LD," Journal of Crystal Growth 300, 2-10 (2007).

54. Y. Ji, Z. H. Zhang, Z. Kyaw, S. T. Tan, Z. G. Ju, X. L. Zhang, W. Liu, X. W. Sun, and H. V. Demir, "Influence of n-type versus p-type AlGaN electronblocking layer on InGaN/GaN multiple quantum wells light-emitting diodes," Appl Phys Lett 103, 053512 (2013).

55. Y. Ji, Z. H. Zhang, S. T. Tan, Z. G. Ju, Z. Kyaw, N. Hasanov, W. Liu, X. W. Sun, and H. V. Demir, "Enhanced hole transport in InGaN/GaN multiple quantum well light-emitting diodes with a p-type doped quantum barrier," Opt Lett 38, 202-204 (2013).

56. Z. G. Ju, W. Liu, Z. H. Zhang, S. T. Tan, Y. Ji, Z. B. Kyaw, X. L. Zhang, S. P. Lu, Y. P. Zhang, B. B. Zhu, N. Hasanov, X. W. Sun, and H. V. Demir, "Improved hole distribution in InGaN/GaN light-emitting diodes with graded thickness quantum barriers," Appl Phys Lett 102, 243504 (2013).

57. Z. H. Zhang, S. T. Tan, Y. Ji, W. Liu, Z. Ju, Z. Kyaw, X. W. Sun, and H. V. Demir, "A PN-type quantum barrier for InGaN/GaN light emitting diodes," Opt Express 21, 15676-15685 (2013).

58. Z. H. Zhang, S. T. Tan, Z. G. Ju, W. Liu, Y. Ji, Z. Kyaw, Y. Dikme, X. W. Sun, and H. V. Demir, "On the Effect of Step-Doped Quantum Barriers in InGaN/GaN Light Emitting Diodes," J Disp Technol 9, 226-233 (2013).

59. Z. H. Zhang, S. T. Tan, W. Liu, Z. G. Ju, K. Zheng, Z. Kyaw, Y. Ji, N. Hasanov, 
X. W. Sun, and H. V. Demir, "Improved InGaN/GaN light-emitting diodes with a p-GaN/n-GaN/p-GaN/n-GaN/p-GaN current-spreading layer," Opt Express 21, 4958-4969 (2013).

60. M.-H. Kim, M. F. Schubert, Q. Dai, J. K. Kim, E. F. Schubert, J. Piprek, and Y. Park, "Origin of efficiency droop in GaN-based light-emitting diodes," Appl Phys Lett 91, 183507 (2007).

61. T. Shinagawa, Y. Abe, H. Matsumoto, B. C. Li, K. Murakami, N. Okada, K. Tadatomo, M. Kannaka, and H. Fujii, "Light-emitting diodes fabricated on nanopatterned sapphire substrates by thermal lithography," Physica Status Solidi C: Current Topics in Solid State Physics 7, 2165-2167 (2010).

62. S. Nakamura, T. Mukai, and M. Senoh, "High-Power GaN P-N Junction BlueLight-Emitting Diodes," Japanese Journal of Applied Physics 30, L1998 (1991).

63. S. Nakamura, M. Senoh, and T. Mukai, "P-GaN/N-InGaN/N-GaN DoubleHeterostructure Blue-Light-Emitting Diodes," Japanese Journal of Applied Physics 32, L8 (1993).

64. S. Nakamura, M. Senoh, and T. Mukai, "High-power InGaN/GaN doubleheterostructure violet light emitting diodes," Applied Physics Letters 62, 23902392 (1993).

65. T. Kazuyuki, O. Hiroaki, O. Youichiro, T. Takashi, I. Yoshiyuki, K. Munehiro, and T. Tsunemasa, "High Output Power InGaN Ultraviolet Light-Emitting Diodes Fabricated on Patterned Substrates Using Metalorganic Vapor Phase Epitaxy," Japanese Journal of Applied Physics 40, L583 (2001).

66. S. Nakamura, M. Senoh, S. Nagahama, N. Iwasa, T. Yamada, T. Matsushita, H. Kiyoku, Y. Sugimoto, T. Kozaki, H. Umemoto, M. Sano and K. Chocho, "High-Power, Long-Lifetime InGaN/GaN/AlGaN-Based Laser Diodes Grown on Pure GaN Substrates," Japanese Journal of Applied Physics 37, L309 (1998).

67. S. Nagahama, N. Iwasa, M. Senoh, T. Matsushita, Y. Sugimoto, H. Kiyoku, T. Kozaki, M. Sano, H. Matsumura, H. Umemoto, K. Chocho and T. Mukai, "High-Power and Long-Lifetime InGaN Multi-Quantum-Well Laser Diodes Grown on Low-Dislocation-Density GaN Substrates," Japanese Journal of Applied Physics 39, L647 (2000).

68. S. Nakamura, M. Senoh, S. Nagahama, N. Iwasa, T. Yamada, T. Matsushita, Y. Sugimoto and H. Kiyoku, "High-Power, Long-Lifetime InGaN Multi- 
Quantum-Well-Structure Laser Diodes," Japanese Journal of Applied Physics 36, L1059 (1997).

69. Y. F. Wu, B. P. Keller, S. Keller, D. Kapolnek, P. Kozodoy, S. P. Denbaars, and U. K. Mishra, "Very high breakdown voltage and large transconductance realized on GaN heterojunction field effect transistors," Applied Physics Letters 69, 1438-1440 (1996).

70. M. A. Khan, X. Hu, G. Sumin, A. Lunev, J. Yang, R. Gaska, and M. S. Shur, "AlGaN/GaN metal oxide semiconductor heterostructure field effect transistor," IEEE Electron Device Letters 21, 63-65 (2000).

71. A. T. Ping, Q. Chen, J. W. Yang, M. A. Khan, and I. Adesida, "DC and microwave performance of high-current $\mathrm{AlGaN} / \mathrm{GaN}$ heterostructure field effect transistors grown on p-type SiC substrates," IEEE Electron Device Letters 19, 54-56 (1998).

72. D. Li, X. Sun, H. Song, Z. Li, Y. Chen, H. Jiang, and G. Miao, "Realization of a High-Performance GaN UV Detector by Nanoplasmonic Enhancement," Advanced Materials 24, 845-849 (2012).

73. H. Morkoç, A. D. Carlo, and R. Cingolani, "GaN-based modulation doped FETs and UV detectors," Solid-State Electronics 46, 157-202 (2002).

74. O. Siegmund, J. Vallerga, J. McPhate, J. Malloy, A. Tremsin, A. Martin, M. Ulmer, and B. Wessels, "Development of GaN photocathodes for UV detectors," Nuclear Instruments and Methods in Physics Research Section A: Accelerators, Spectrometers, Detectors and Associated Equipment 567, 89-92 (2006).

75. E. Matioli, C. Neufeld, M. Iza, S. C. Cruz, A. A. Al-Heji, X. Chen, R. M. Farrell, S. Keller, S. DenBaars, U. Mishra, S. Nakamura, J. Speck, and C. Weisbuch, "High internal and external quantum efficiency InGaN/GaN solar cells," Applied Physics Letters 98, 021102 (2011).

76. O. Jani, I. Ferguson, C. Honsberg, and S. Kurtz, "Design and characterization of GaNnGaN solar cells," Applied Physics Letters 91, 132117 (2007).

77. J. H. Lee, D. Y. Lee, B. W. Oh, and J. H. Lee, "Comparison of InGaN-Based LEDs Grown on Conventional Sapphire and Cone-Shape-Patterned Sapphire Substrate," IEEE Transactions on Electron Devices 57, 157-163 (2010).

78. Y. J. Lee, C. H. Chiu, C. C. Ke, P. C. Lin, T. C. Lu, H. C. Kuo, and S. C. Wang, "Study of the Excitation Power Dependent Internal Quantum Efficiency in InGaN/GaN LEDs Grown on Patterned Sapphire Substrate," IEEE Journal of 
Selected Topics in Quantum Electronics 15, 1137-1143 (2009).

79. J. Edmond, A. Abare, M. Bergman, J. Bharathan, K. Lee Bunker, D. Emerson, K. Haberern, J. Ibbetson, M. Leung, P. Russel, and D. Slater, "High efficiency GaN-based LEDs and lasers on SiC," Journal of Crystal Growth 272, 242-250 (2004).

80. M. E. Lin, S. Strite, A. Agarwal, A. Salvador, G. L. Zhou, N. Teraguchi, A. Rockett, and H. Morkoç, "GaN grown on hydrogen plasma cleaned $6 \mathrm{H}-\mathrm{SiC}$ substrates," Applied Physics Letters 62, $702-704$ (1993).

81. K. Akihiko, K. Mizue, T. Makoto, and K. Katsumi, "InGaN/GaN Multiple Quantum Disk Nanocolumn Light-Emitting Diodes Grown on (111) Si Substrate," Japanese Journal of Applied Physics 43, L1524 (2004).

82. A. Krost, and A. Dadgar, "GaN-based optoelectronics on silicon substrates," Materials Science and Engineering: B 93, 77-84 (2002).

83. C. Xiong, F. Jiang, W. Fang, L. Wang, C. Mo, and H. Liu, "The characteristics of GaN-based blue LED on Si substrate," Journal of Luminescence 122-123, 185-187 (2007).

84. F. Mitsuru, U. Masaya, K. Yoichi, N. Yukio, K. Takao, T. Masayoshi, and M. Takashi, "Blue, Green, and Amber InGaN/GaN Light-Emitting Diodes on Semipolar \{11-22\} GaN Bulk Substrates," Japanese Journal of Applied Physics 45, L659 (2006).

85. T. Nishida, H. Saito, and N. Kobayashi, "Efficient and high-power AlGaNbased ultraviolet light-emitting diode grown on bulk GaN," Applied Physics Letters 79, 711-712 (2001).

86. S. Fritze, A. Dadgar, H. Witte, M. Bügler, A. Rohrbeck, J. Bläsing, A. Hoffmann, and A. Krost, "High Si and Ge n-type doping of GaN doping Limits and impact on stress," Applied Physics Letters 100, 122104 (2012).

87. F. Chris, and L. William, "The dependence of Raman scattering on $\mathrm{Mg}$ concentration in Mg-doped GaN grown by MBE," Materials Research Express 1, 025901 (2014).

88. Wikipedia, "Gallium nitride," https://en.wikipedia.org/wiki/Gallium_nitride, 2016 (Accessed in June 2016).

89. J. Han, M. H. Crawford, R. J. Shul, J. J. Figiel, M. Banas, L. Zhang, Y. K. Song, H. Zhou, and A. V. Nurmikko, "AlGaN/GaN quantum well ultraviolet light emitting diodes," Applied Physics Letters 73, 1688-1690 (1998).

90. S.-H. Han, D.-Y. Lee, S.-J. Lee, C.-Y. Cho, M.-K. Kwon, S. P. Lee, D. Y. Noh, 
D.-J. Kim, Y. C. Kim, and S.-J. Park, "Effect of electron blocking layer on efficiency droop in InGaN/GaN multiple quantum well light-emitting diodes," Applied Physics Letters 94, 231123 (2009).

91. D. F. Feezell, M. C. Schmidt, S. P. DenBaars, and S. Nakamura, "Development of Nonpolar and Semipolar InGaN/GaN Visible Light-Emitting Diodes," MRS Bulletin 34, 318-323 (2009).

92. S. D. Lester, M. J. Ludowise, K. P. Killeen, B. H. Perez, J. N. Miller, and S. J. Rosner, "High-efficiency InGaN MQW blue and green LEDs," Journal of Crystal Growth 189-190, 786-789 (1998).

93. E. F. Schubert, "Radiative and non-radiative recombination," in Light-Emitting Diodes(Cambridge University Press), 27-47 (2006).

94. E. F. Schubert, T. Gessmann, and J. K. Kim, "Light Emitting Diodes," in KirkOthmer Encyclopedia of Chemical Technology(John Wiley \& Sons, Inc., 2000).

95. L. Wei, Z. Degang, J. Desheng, C. Ping, L. Zongshun, Z. Jianjun, L. Xiang, L. Feng, L. Jianping, Z. Liqun, Y. Hui, Z. Yuantao, and D. Guotong, "ShockleyRead-Hall recombination and efficiency droop in InGaN/GaN multiplequantum-well green light-emitting diodes," Journal of Physics D: Applied Physics 49, 145104 (2016).

96. J. Peretti, C. Weisbuch, J. Iveland, M. Piccardo, L. Martinelli, and J. S. Speck, "Identification of Auger effect as the dominant mechanism for efficiency droop of LEDs," pp. 90030Z-90030Z-90014 (2014).

97. E. Kioupakis, P. Rinke, K. T. Delaney, and C. G. Van de Walle, "Indirect Auger recombination as a cause of efficiency droop in nitride light-emitting diodes," Applied Physics Letters 98, 161107 (2011).

98. F. Römer, and B. Witzigmann, "Effect of Auger recombination and leakage on the droop in InGaN/GaN quantum well LEDs," Opt. Express 22, A1440-A1452 (2014).

99. J. Senawiratne, A. Chatterjee, T. Detchprohm, W. Zhao, Y. Li, M. Zhu, Y. Xia, X. Li, J. Plawsky, and C. Wetzel, "Junction temperature, spectral shift, and efficiency in GaInN-based blue and green light emitting diodes," Thin Solid Films 518, 1732-1736 (2010).

100. Q. Dai, Q. Shan, J. Wang, S. Chhajed, J. Cho, E. F. Schubert, M. H. Crawford, D. D. Koleske, M.-H. Kim, and Y. Park, "Carrier recombination mechanisms and efficiency droop in GaInN/GaN light-emitting diodes," Appl. Phys. Lett 
97, 133507 (2010).

101. D. S. Meyaard, G.-B. Lin, Q. Shan, J. Cho, E. Fred Schubert, H. Shim, M.-H. Kim, and C. Sone, "Asymmetry of carrier transport leading to efficiency droop in GaInN based light-emitting diodes," Applied Physics Letters 99, 251115 (2011).

102. G. W. Marsh, and J. M. Kahn, "50-Mb/s diffuse infrared free-space link using on-off keying with decision-feedback equalization," IEEE Photonics Technology Letters 6, 1268-1270 (1994).

103. L. Zeng, D. C. O'Brien, H. L. Minh, G. E. Faulkner, K. Lee, D. Jung, Y. Oh, and E. T. Won, "High data rate multiple input multiple output (MIMO) optical wireless communications using white LED lighting," IEEE J.Sel. A. Commun. 27, 1654-1662 (2009).

104. K. Cui, J. Quan, and Z. Xu, "Performance of indoor optical femtocell by visible light communication," Optics Communications 298-299, 59-66 (2013).

105. J. W. Shi, J. K. Sheu, C. H. Chen, G. R. Lin, and W. C. Lai, "High-Speed GaNBased Green Light-Emitting Diodes With Partially n-Doped Active Layers and Current-Confined Apertures," IEEE Electron Device Letters 29, 158-160 (2008).

106. A. J. Shaw, A. L. Bradley, J. F. Donegan, and J. G. Lunney, "GaN resonant cavity light-emitting diodes for plastic optical fiber applications," IEEE Photonics Technology Letters 16, 2006-2008 (2004).

107. S. Lu, W. Liu, Z.-H. Zhang, S. T. Tan, Z. Ju, Y. Ji, X. Zhang, Y. Zhang, B. Zhu, Z. Kyaw, N. Hasanov, X. W. Sun, and H. V. Demir, "Low thermal-mass LEDs: size effect and limits," Opt Express 22, 32200-32207 (2014).

108. W. E. Howard, and O. F. Prache, "Microdisplays based upon organic lightemitting diodes," IBM Journal of Research and Development 45, 115-127 (2001).

109. L. Magazine, "Aixtron MOCVD system is selected by Vishay Semiconductor for expanded infrared LED production," http://www.ledsmagazine.com/ugc/2014/08/01/aixtron-mocvd-system-isselected-by-vishay-semiconductor-for-expanded-infrared-ledproduction.html, 2014 (Accessed in April 2016).

110. $\mathrm{P}$

Media,

"Showerhead Reactor," http://www.photonics.com/Product.aspx?PRID=42290, 2016 (Accessed in April 2016). 
111. M. R. Krames, O. B. Shchekin, R. Mueller-Mach, G. O. Mueller, Z. Ling, G. Harbers, and M. G. Craford, "Status and Future of High-Power Light-Emitting Diodes for Solid-State Lighting," Display Technology, Journal of 3, 160-175 (2007).

112. S. T. Tan, X. W. Sun, H. V. Demir, and S. P. DenBaars, "Advances in the LED Materials and Architectures for Energy-Saving Solid-State Lighting Toward "Lighting Revolution"," IEEE Photonics J 4, 613-619 (2012).

113. H. W. Choi, C. W. Jeon, and M. D. Dawson, "InGaN microring light-emitting diodes," IEEE Photonic Tech L 16, 33-35 (2004).

114. V. Fiorentini, F. Bernardini, and O. Ambacher, "Evidence for nonlinear macroscopic polarization in III-V nitride alloy heterostructures," Appl Phys Lett 80, 1204-1206 (2002).

115. H. P. Zhao, R. A. Arif, Y. K. Ee, and N. Tansu, "Self-Consistent Analysis of Strain-Compensated InGaN-AlGaN Quantum Wells for Lasers and LightEmitting Diodes," IEEE J Quantum Elect 45, 66-78 (2009).

116. M. Meneghini, N. Trivellin, G. Meneghesso, E. Zanoni, U. Zehnder, and B. Hahn, "A combined electro-optical method for the determination of the recombination parameters in InGaN-based light-emitting diodes," Journal of Applied Physics 106, 114508 (2009).

117. J. Piprek, and S. Nakamura, "Physics of high-power InGaN/GaN lasers," Iee P-Optoelectron 149, 145-151 (2002).

118. I. Vurgaftman, and J. R. Meyer, "Band parameters for nitrogen-containing semiconductors," Journal of Applied Physics 94, 3675-3696 (2003).

119. K. A. Bulashevich, I. Y. Evstratov, V. F. Mymrin, and S. Y. Karpov, "Current spreading and thermal effects in blue LED dice," Phys Status Solidi C 4, 45-48 (2007).

120. Y. P. Zhang, Z.-H. Zhang, W. Liu, S. T. Tan, Z. G. Ju, X. L. Zhang, Y. Ji, L. C. Wang, Z. Kyaw, N. Hasanov, B. B. Zhu, S. P. Lu, X. W. Sun, and H. V. Demir, "Nonradiative recombination - critical in choosing quantum well number for InGaN/GaN light-emitting diodes," Opt Express 23, A34-A42 (2015).

121. T. Taguchi, Y. Uchida, and K. Kobashi, "Efficient white LED lighting and its application to medical fields," physica status solidi (a) 201, 2730-2735 (2004).

122. A. Jovicic, L. Junyi, and T. Richardson, "Visible light communication: opportunities, challenges and the path to market," IEEE Communications Magazine 51, 26-32 (2013). 
123. J. C. Rojas, and F. Gonzalez-Lima, "Neurological and psychological applications of transcranial lasers and LEDs," Biochemical Pharmacology 86, 447-457 (2013).

124. J. Y. Kim, M. K. Kwon, J. P. Kim, and S. J. Park, "Enhanced light extraction from triangular GaN-Based light-emitting diodes," IEEE Photonic Tech L 19, 1865-1867 (2007).

125. X. H. Wang, W. Y. Fu, P. T. Lai, and H. W. Choi, "Evaluation of InGaN/GaN light-emitting diodes of circular geometry," Opt Express 17, 22311-22319 (2009).

126. X. H. Wang, P. T. Lai, and H. W. Choi, "The contribution of sidewall light extraction to efficiencies of polygonal light-emitting diodes shaped with laser micromachining," Journal of Applied Physics 108, 023110 (2010).

127. S. C. Chung, P. C. Ho, D. R. Li, T. X. Lee, T. H. Yang, and C. C. Sun, "Effect of chip spacing on light extraction for light-emitting diode array," Opt Express 23, A640-649 (2015).

128. K. A. Bulashevich, I. Y. Evstratov, V. F. Mymrin, and S. Y. Karpov, "Current spreading and thermal effects in blue LED dice," physica status solidi (c) 4, 4548 (2007).

129. Z.-H. Zhang, S. Tiam Tan, Z. Kyaw, Y. Ji, W. Liu, Z. Ju, N. Hasanov, X. Wei Sun, and H. Volkan Demir, "InGaN/GaN light-emitting diode with a polarization tunnel junction," Applied Physics Letters 102, 193508 (2013).

130. J. H. Ryou, P. D. Yoder, J. Liu, Z. Lochner, H. Kim, S. Choi, H. J. Kim, and R. D. Dupuis, "Control of Quantum-Confined Stark Effect in InGaN-Based Quantum Wells," IEEE Journal of Selected Topics in Quantum Electronics 15, 1080-1091 (2009).

131. T. Tetsuya, S. Shigetoshi, K. Maki, K. Miho, T. Hideo, A. Hiroshi, and A. Isamu, "Quantum-Confined Stark Effect due to Piezoelectric Fields in GaInN Strained Quantum Wells," Japanese Journal of Applied Physics 36, L382 (1997).

132. P. Waltereit, O. Brandt, A. Trampert, H. T. Grahn, J. Menniger, M. Ramsteiner, M. Reiche, and K. H. Ploog, "Nitride semiconductors free of electrostatic fields for efficient white light-emitting diodes," Nature 406, 865-868 (2000).

133. H.-M. Kim, Y.-H. Cho, H. Lee, S. I. Kim, S. R. Ryu, D. Y. Kim, T. W. Kang, and K. S. Chung, "High-Brightness Light Emitting Diodes Using DislocationFree Indium Gallium Nitride/Gallium Nitride Multiquantum-Well Nanorod 
Arrays," Nano Letters 4, 1059-1062 (2004).

134. A. Chakraborty, B. A. Haskell, S. Keller, J. S. Speck, S. P. Denbaars, S. Nakamura and U. K. Mishra, "Demonstration of Nonpolar m -Plane InGaN/GaN Light-Emitting Diodes on Free-Standing $m$-Plane GaN Substrates," Japanese Journal of Applied Physics 44, L173 (2005).

135. S. P. DenBaars, D. Feezell, K. Kelchner, S. Pimputkar, C.-C. Pan, C.-C. Yen, S. Tanaka, Y. Zhao, N. Pfaff, R. Farrell, M. Iza, S. Keller, U. Mishra, J. S. Speck, and S. Nakamura, "Development of gallium-nitride-based lightemitting diodes (LEDs) and laser diodes for energy-efficient lighting and displays," Acta Materialia 61, 945-951 (2013).

136. Y. Tian, Y. Shao, Y. Wu, X. Hao, L. Zhang, Y. Dai, and Q. Huo, "Direct growth of freestanding GaN on C-face SiC by HVPE," Scientific reports 5 (2015).

137. V. Y. Davydov, N. Averkiev, I. Goncharuk, D. Nelson, I. Nikitina, A. Polkovnikov, A. Smirnov, M. Jacobson, and O. Semchinova, "Raman and photoluminescence studies of biaxial strain in GaN epitaxial layers grown on 6H-SiC," Journal of applied physics 82, 5097-5102 (1997).

138. S. Tripathy, R. K. Soni, H. Asahi, K. Iwata, R. Kuroiwa, K. Asami, and S. Gonda, "Optical properties of GaN layers grown on C-, A-, R-, and M-plane sapphire substrates by gas source molecular beam epitaxy," Journal of Applied Physics 85, 8386-8399 (1999).

139. V. Y. Davydov, Y. E. Kitaev, I. Goncharuk, A. Smirnov, J. Graul, O. Semchinova, D. Uffmann, M. Smirnov, A. Mirgorodsky, and R. Evarestov, "Phonon dispersion and Raman scattering in hexagonal GaN and AlN," Physical Review B 58, 12899 (1998).

140. S. Tripathy, S. Chua, P. Chen, and Z. Miao, "Micro-Raman investigation of strain in $\mathrm{GaN}$ and AlxGa1- xN/GaN heterostructures grown on Si (111)," Journal of Applied Physics 92, 3503-3510 (2002).

141. Z. G. Ju, S. T. Tan, Z.-H. Zhang, Y. Ji, Z. Kyaw, Y. Dikme, X. W. Sun, and H. V. Demir, "On the origin of the redshift in the emission wavelength of InGaN/GaN blue light emitting diodes grown with a higher temperature interlayer," Applied Physics Letters 100, 123503 (2012).

142. M. C. Johnson, E. D. Bourret-Courchesne, J. Wu, Z. Liliental-Weber, D. N. Zakharov, R. J. Jorgenson, T. B. Ng, D. E. McCready, and J. R. Williams, "Effect of gallium nitride template layer strain on the growth of InxGa1$\mathrm{xN} / \mathrm{GaN}$ multiple quantum well light emitting diodes," Journal of Applied 
Physics 96, 1381-1386 (2004).

143. X. A. Cao, P. M. Sandvik, S. F. LeBoeuf, and S. D. Arthur, "Defect generation in InGaN/GaN light-emitting diodes under forward and reverse electrical stresses," Microelectronics Reliability 43, 1987-1991 (2003).

144. J. J. Chen, Y. K. Su, C. L. Lin, S. M. Chen, W. L. Li, and C. C. Kao, "Enhanced Output Power of GaN-Based LEDs With Nano-Patterned Sapphire Substrates," IEEE Photonic Tech L 20, 1193-1195 (2008).

145. P.-H. Chen, V.-C. Su, M.-L. Lee, Y.-H. You, Y.-P. Chen, Z.-H. Hung, T.-C. Hsu, Y.-Y. Lin, R.-M. Lin, and C.-H. Kuan, "Strain Relaxation in InGaN/GaN Multiple-Quantum Wells by Nano-Patterned Sapphire Substrates with Smaller Period," in CLEO: 2015(Optical Society of America, San Jose, California), p. JTh2A.31 (2015).

146. P. Altieri-Weimar, A. Jaeger, T. Lutz, P. Stauss, K. Streubel, K. Thonke, and R. Sauer, "Influence of doping on the reliability of AlGaInP LEDs," Journal of Materials Science: Materials in Electronics 19, 338-341 (2008). 